\title{
Pig tail biting in different farrowing and rearing systems with a focus on tail lesions, tail losses and activity monitoring
}

Dissertation to attain the doctoral degree (Dr. sc. agr.) of the Faculty of Agricultural Sciences Georg-August-Universität Göttingen Submitted by

Maria Gentz born on the $3^{\text {rd }}$ May 1992 in Bad Oldesloe 
1. Referee: Prof. Dr. Imke Traulsen

2. Referee:Prof. Dr. Jürgen Hummel

3. Referee: Prof. Dr. Eva Schlecht

Date of oral examination: $9^{\text {th }}$ July 2020 



\section{TABLE OF CONTENTS}

$\begin{array}{ll}\text { GENERAL INTRODUCTION } & 1\end{array}$

LITERATURE REVIEW - BACKGROUND OF TAIL BITING 6

PAPER ONE

Tail lesions and losses of docked and undocked pigs in different farrowing and rearing systems

PAPER TWO

Classification of pigs with tail lesions from different farrowing and rearing systems during rearing and fattening period

\section{PAPER THREE}

Different housing systems during nursery: tail lesions and losses, skin lesions and performance during rearing and fattening period

PAPER FOUR

Activity behaviour of pigs: comparison of manual and automated video analyses 


\section{GENERAL INTRODUCTION}

Tail biting is a widespread behavioural disorder in modern pig housing systems (SchrøderPetersen and Simonsen, 2001). This abnormal behaviour was already known at the beginning of the 19th century (Sambraus, 1985), where cold stable climate and a lack of bedding were detected as causes, but it only became a problem in the 1950s (Schrøder-Petersen and Simonsen, 2001). The prevalence of tail lesions on pig farms ranges from 25 to $75 \%$ depending on docking status, age and housing, and management conditions (Larsen et al., 2018; Naya et al., 2018; Ursinus et al., 2014; Valros et al., 2004; Veit et al., 2016). Although research has been going on for many years, no solution has been found to prevent tail biting so far.

In general, tail biting describes the oral manipulation of a pig's tail by another. Different types of tail biting are described throughout literature which are discernible by the method of biting as well as multifactorial causes (Taylor et al., 2010). Tail biting results in tail lesions that range from tooth imprints to lesions with or without inflammations (Schrøder-Petersen and Simonsen, 2001). One of the most common methods to reduce tail biting behaviour and tail lesions, is tail docking. By docking the tail, a higher sensitivity in the remaining part of the tail is reached and additionally, it is harder for the pigs to take hold of (Simonsen et al., 1991). Although routine tail docking is banned in the EU (Council of European Union, 2008), it is still used. The acute welfare risks of this amputation such as the development of neuroanatomical changes, e.g. the formation of neuromas (Herskin et al., 2015), seem to be less important than the long-term welfare risks from tail biting (Nannoni et al., 2014). Nevertheless, the reduction of risk factors seems to be the more sustainable strategy against this abnormal behaviour, particularly with regard to animal welfare (Nannoni et al., 2014). Tail biting has multifactorial causes and many factors need to be considered to reduce it. A distinction between acute and chronic risk factors is required (Valros, 2018). The chronic factors include the housing system (e.g. SchrøderPetersen and Simonsen, 2001), feeding (e.g. Valros et al., 2016), space allowance (e.g. Munsterhjelm et al., 2017), sex (e.g. Zonderland et al., 2010b) and genetics (e.g. Breuer et al., 2005). Acute risk factors comprise sudden changes in the environment of the pig, such as changes in temperature or air quality or interruptions to the normal feeding pattern due to feeder malfunction (Valros, 2018). The possible consequences of tail biting affect animal welfare due to the fact that the pig may partly or completely lose the tail (Veit et al., 2016) and suffer from pain (Harley et al., 2014). Furthermore, it can reduce production efficiency, increase on-farm costs (e.g. labour and treatment costs) and lead to a variety of physical lesions and carcass condemnation resulting in financial losses for the farmer and the abattoir (Harley et al., 2014; Zonderland, 2010a). 
Tail biting and its risk factors are well known in pig husbandry; studies have shown that it can especially be reduced by housing and management decisions (EFSA, 2007). The development of a suitable farrowing system for piglets, sow and farmer is already challenging (Baxter et al., 2011). There are various conflicts of interest of animal needs and economic concerns, such as space for the sow, nest building and piglet survival, which need to be solved by appropriate pen design (Baxter et al., 2011). Raising piglets in systems like a conventional farrowing crate can influence the subsequent cognitive abilities of pigs (de Jong et al., 2000) and their ability to cope with stressful situations (de Jonge, 1996). Moreover, the rearing environment is important in the development of abnormal behaviour patterns (Schrøder-Petersen and Simonsen, 2001). Pigs that are housed with less stress have a lower frequency of tail biting than pigs reared in conventional systems (Ekkel et al., 1995). Furthermore, an increased space allowance reduces the number of tail lesions (Munsterhjelm et al., 2015).

\section{The aim of the present dissertation}

The aim of the present dissertation was to evaluate the effect of different farrowing and rearing systems on tail lesions and losses of rearing and fattening pigs. The focus was to investigate the differences of docked and undocked animals. The farrowing systems differed in the possibilities to provide social interaction of the piglets among each other and with the sows. The rearing systems differed regarding the management of regrouping, the space allowance and the composition of the groups (single sex/mixed sex).

Paper One describes the differences in tail lesions and losses of docked and undocked pigs during rearing and fattening period. The pigs were housed in one of three farrowing and two rearing systems. A weekly tail scoring, which started after weaning, showed the development of tail lesions and losses.

Paper Two examines the animal individual differences of pigs with tail lesions. Different intensities of lesions were weighted by a lesion parameter and grouped afterwards. In a subsequent cluster analysis, the different characteristics of animals without or with a high occurrence of lesions were identified.

Paper Three investigates the long-term effects of artificial rearing during the nursery period. The analysis focused on tail lesions and losses, skin lesions and performance traits. The motherless rearing system was compared to a conventional farrowing crate.

Paper Four deals with a comparison of two video analysis methods. The activity behaviour of rearing pigs, which might be a predictor for tail biting, was evaluated by a manual as well as by automated video image analysis. 


\section{References}

Baxter, E.M., Lawrence, A.B., Edwards, S.A., 2011. Alternative farrowing systems: design criteria for farrowing systems based on the biological needs of sows and piglets. Animal 5 (4), 580-600. https://doi.org/10.1017/S1751731110002272.

Breuer, K., Sutcliffe, M.E.M., Mercer, J.T., Rance, K.A., O'Connell, N.E., Sneddon, I.A., Edwards, S.A., 2005. Heritability of clinical tail-biting and its relation to performance traits. Livestock Production Science 93 (1), 87-94. https://doi.org/10.1016/j.livprodsci.2004.11.009.

Council of European Union, 2008. Council directive 2008/120/EC.

de Jong, I., Prelle, I., Vandenburgwal, J., Lambooij, E., Korte, S., Blokhuis, H., Koolhaas, J., 2000. Effects of environmental enrichment on behavioral responses to novelty, learning, and memory, and the circadian rhythm in cortisol in growing pigs. Physiology \& Behavior 68 (4), 571-578. https://doi.org/10.1016/S0031-9384(99)00212-7.

de Jonge, F., 1996. Rearing Piglets in a Poor Environment: Developmental Aspects of Social Stress in Pigs. Physiology \& Behavior 60 (2), 389-396. https://doi.org/10.1016/00319384(96)00013-3.

EFSA, 2007. European Food Safety Authority: Scientific Opinion of the Panel on Animal Health and Welfare on a request from commission on the risks associated with tail biting in pigs and possible means to reduce the need for tail docking considering the different housing and husbandry systems, EFSA J. 611, 1-13.

Ekkel, E.D., van Doorn, C.E., Hessing, M.J., Tielen, M.J., 1995. The Specific-Stress-Free housing system has positive effects on productivity, health, and welfare of pigs. Journal of Animal Science 73 (6), 1544-1551. https://doi.org/10.2527/1995.7361544x.

Harley, S., Boyle, L., O'Connell, N.E., More, S.J., Teixeira, D.L., Hanlon, A., 2014. Docking the value of pig meat? Prevalence and financial implications of welfare lesions in Irish slaughter pigs. Animal Welfare 23 (3), 275-285. https://doi.org/10.7120/09627286.23.3.275.

Herskin, M.S., Thodberg, K., Jensen, H.E., 2015. Effects of tail docking and docking length on neuroanatomical changes in healed tail tips of pigs. Animal 9 (4), 677-681. https://doi.org/10.1017/S1751731114002857.

Larsen, M.L.V., Andersen, H.M.-L., Pedersen, L.J., 2018. Which is the most preventive measure against tail damage in finisher pigs: tail docking, straw provision or lowered stocking density? Animal 12 (6), 1260-1267. https://doi.org/10.1017/S175173111700249X.

Munsterhjelm, C., Heinonen, M., Valros, A., 2015. Application of the Welfare Quality ${ }^{\circledR}$ animal welfare assessment system in Finnish pig production, part II: Associations between 
animal-based and environmental measures of welfare. Animal Welfare 24 (2), 161-172. https://doi.org/10.7120/09627286.24.2.161.

Munsterhjelm, C., Nordgreen, J., Aae, F., Heinonen, M., Olstad, K., Aasmundstad, T., Janczak, A.M., Valros, A., 2017. To be blamed or pitied? The effect of illness on social behavior, cytokine levels and feed intake in undocked boars. Physiology \& Behavior 179, 298-307. https://doi.org/10.1016/j.physbeh.2017.06.024.

Nannoni, E., Valsami, T., Sardi, L., Martelli, G., 2014. Tail Docking in Pigs: A Review on its Short- And Long-Term Consequences and Effectiveness in Preventing Tail Biting. Italian Journal of Animal Science 13 (1), 3095. https://doi.org/10.4081/ijas.2014.3095.

Naya, A., Traulsen, I., Gertz, M., Hasler, M., Burfeind, O., große Beilage, E., Krieter, J., 2018. Is tail biting in growing pigs reduced by a prolonged suckling period? Applied Animal Behaviour Science. https://doi.org/10.1016/j.applanim.2018.10.020.

Sambraus, H.H., 1985. Mouth-based anomalous syndromes: Ethology of Farm Animals. A Comprehensive Study of the Behavioural Features of Common Farm Animals. World Animal Science A5, 391-422.

Schrøder-Petersen, D.L., Simonsen, H.B., 2001. Tail biting in pigs. Veterinary journal 162 (3), 196-210. https://doi.org/10.1053/tvjl.2001.0605.

Simonsen, H.B., Klinken, L., Bindseil, E., 1991. Histopathology of intact and docked pigtails. British Veterinary Journal 147 (5), 407-412. https://doi.org/10.1016/0007-1935(91)90082$\mathrm{X}$.

Taylor, N.R., Main, D.C.J., Mendl, M., Edwards, S.A., 2010. Tail-biting: A new perspective. Veterinary journal 186 (2), 137-147. https://doi.org/10.1016/j.tvjl.2009.08.028.

Ursinus, W.W., Wijnen, H.J., Bartels, A.C., Dijvesteijn, N., van Reenen, C.G., Bolhuis, J.E., 2014. Damaging biting behaviors in intensively kept rearing gilts: the effect of jute sacks and relations with production characteristics. Journal of Animal Science 92 (11), 51935202. https://doi.org/10.2527/jas.2014-7918.

Valros, A., 2018. Tail biting, in: Špinka, M. (Ed.), Advances in pig welfare. Woodhead Publishing an imprint of Elsevier, Duxford, United Kingdom, 137-166.

Valros, A., Ahlström, S., Rintala, H., Häkkinen, T., Saloniemi, H., 2004. The prevalence of tail damage in slaughter pigs in Finland and associations to carcass condemnations. Acta Agriculturae Scandinavica, Section A - Animal Science 54 (4), 213-219. https://doi.org/10.1080/09064700510009234.

Valros, A., Munsterhjelm, C., Hänninen, L., Kauppinen, T., Heinonen, M., 2016. Managing undocked pigs - on-farm prevention of tail biting and attitudes towards tail biting and docking. Porcine health management 2, 2. https://doi.org/10.1186/s40813-016-0020-7. 
Veit, C., Traulsen, I., Hasler, M., Tölle, K., Burfeind, O., grosse Beilage, E., Krieter, J., 2016. Influence of raw material on the occurrence of tail-biting in undocked pigs. Livestock Science 191, 125-131. https://doi.org/10.1016/j.livsci.2016.07.009.

Zonderland, J.J., 2010a. Talking tails: Quantifying the development of tail biting in pigs. Dissertation. Wageningen University, Wageningen, $176 \mathrm{pp}$.

Zonderland, J.J., Bracke, M.B.M., den Hartog, L.A., Kemp, B., Spoolder, H.A.M., 2010b. Gender effects on tail damage development in single- or mixed-sex groups of weaned piglets. Livestock Science 129 (1-3), 151-158.

https://doi.org/10.1016/j.livsci.2010.01.018. 


\section{LITERATURE REVIEW - BACKGROUND OF TAIL BITING}

Tail biting is an animal welfare problem and much research has been carried out in recent years, especially due to the prohibition of routine docking (Valros, 2018). Most types of farms, including organic (Bracke et al., 2013) or outdoor housing systems (Walker and Bilkei, 2006) are affected as well as all ages from the suckling phase to the end of fattening. Both undocked and docked pigs can receive tail lesions, therefore it is important to take measures to keep the lesions at an acceptable level (Valros, 2018).

A tail biting outbreak is defined in several ways: Beattie et al. (2005) describe it as an "oral manipulation of a pig's tail by another", Statham et al. (2009) use "blood from damaged tail(s) seen in the pen" as definition, Zonderland et al. (2011b) define it as "at least one pig with a tail wound or at least two pigs with bite marks" and Wallenbeck and Keeling (2013) worked with "when the first pig in a pen was treated for tail damage due to tail biting". Usually definitions include oral contact between the mouth of one pig and the tail of another, which causes varying degrees of lesions depending on the definition.

\section{Types of tail biting}

Taylor et al. (2010) divided tail biting into three types: two-stage, sudden-forceful and obsessive. Two-stage tail biting includes the pre-damage phase, where the pigs are usually lying. One animal has the other's tail in its mouth but does not hurt it. Injuring the tail is part of the damaging phase, where they bite, and the tail can bleed (Schrøder-Petersen et al., 2003). The reason for this behaviour is mostly a lack of enrichment material and can be averted by adding chewable material (Beattie et al., 2001; Zonderland et al., 2008) The second type, "sudden forceful", describes an active and usually standing biter (van Putten, 1969). The victim's tail shows wounds and a partial or a complete loss (van Putten, 1969). This type is caused by inadequate access to resources and can be solved by removing the biter or improving access to feeding and water (Moinard et al., 2003). Obsessive tail biting is repeated grabbing and yanking resulting in wounds with partial or complete loss (Beattie et al., 2005). It might be caused by a genetic disposition, but this has not yet been proved.

\section{Prevalence of tail biting on farms}

Tail biting is a widespread behavioural disorder on pig farms. After weaning, tail biting occurs mainly from the middle of the rearing period and then affects up to $72 \%$ the undocked pigs (Naya et al., 2018; Veit, 2016). Zonderland et al. (2010) investigated how many rearing days are needed until $40 \%$ of the pigs suffered tail lesions and found an average of 16 days. Regarding the fattening period, Larsen et al. (2018) compared the incidence of tail lesions for 
docked pigs (28.33\%), where one third of the group was affected, and undocked pigs (73.08 $\%$ ), where more than two thirds of the animals had a lesion. Ursinus et al. (2014b) investigated the prevalence of tail lesions in sows and they detected that $30 \%$ of the pigs had tail wounds. Concerning the prevalence of tail lesions on abattoirs, pigs operated by 479 farms in Finland were evaluated and produced a value of $34.6 \%$ injured tails (Valros et al., 2004). Although the prevalence of tail biting is high, there is no general monitoring system for the level of tail biting in the European Union so far (Spoolder et al., 2011).

\section{Spread of tail biting in a pen}

Tail biting starts with one biting pig in the pen and can spread very quickly (D'Eath et al., 2014). Small bite marks develop into clearly visible lesions on average within seven days (Zonderland et al., 2011a). The spread can involve an existing victim or different pigs in the pen can be bitten. Moreover, several biters can be identified while copying the biting behaviour of the first one (Blackshaw, 1981), while the number of new biters can vary (D'Eath et al., 2014). Tail biting outbreaks are comparable to a disease and can occur in a sporadic way (Blackshaw, 1981).

\section{Prevention of tail biting}

Tail docking is used to prevent tail biting ( Nannoni et al., 2014; Schrøder-Petersen and Simonsen, 2001). It is a procedure where the length of the tail is reduced by using side-pliers, scissors, a scalpel blade, or gas-/electrical cautery iron while the remaining stump is supposed to be long enough to cover the vulva in females or the equivalent length in males (Sutherland, 2015). Tail docking causes acute pain; neuromas and abscesses can occur which cause chronical pain and discomfort over longer time (Herskin et al., 2015). In addition to pain, the docked animals also show changes in behaviour, including reduced explorative behaviour (Scollo et al., 2013). Previous studies have shown a reduction in tail biting by docking but prevention could not be achieved ( Moinard et al., 2003; Schrøder-Petersen and Simonsen, 2001; Sutherland, 2015).

Since routine tail docking is prohibited in the European Union (Council of European Union, 2008) there is a need to prevent tail biting outbreaks without docking (Larsen et al., 2016). One way of avoiding tail biting is animal monitoring and the detection of indicators for the prediction of future tail biting such as behavioural changes (Larsen et al., 2016) (see section Prediction of tail biting). Early detection would allow a reaction of the farmer, who could possibly avert tail biting activity. Another method is to eliminate the risk factors which cause the problem (EFSA, 2007; Taylor et al., 2012). Nevertheless, tail biting is a multifactorial problem and the elimination of one factor is not sufficient (Schrøder-Petersen and Simonsen, 2001). 


\section{Risk factors for tail biting}

Although there are risk factors which affect the start, the triggering causes can be mixed, which makes it difficult to determine the actual reason (D'Eath et al., 2014). Under natural conditions, pigs spend most of their active phases in search of feed by rooting and exploring (Studnitz et al., 2007). They are omnivorous and move over long distances to satisfy their specific needs (Studnitz et al., 2007). Today, animals are kept under completely different conditions, but they still spend about three quarters of the day in the light periods foraging and exploring the surroundings (Stolba and Wood-Gush, 1980). Hence, domesticated pigs are challenged to fill their available time with a limited number of behavioural patterns compared to wild animals which allocate different behaviours within a limited amount of time, thereby making sure they fulfil needs such as feeding and reproducing (Hughes and Duncan, 1988). Due to the limited conditions in indoor housing systems, the risk factors leading to tail biting are multifactorial (D'Eath et al., 2014). The risks can be divided into chronic and acute factors (Valros, 2018).

\section{Chronic risk factors}

Chronic factors comprise, inter alia, housing system, feeding, space allowance, sex and genetics. They are based on housing and management routines of a farm, though they are more predictable than acute factors (Valros, 2018). In addition, it is often not possible to change fundamental things (e.g. continuous providing of straw) in indoor housing systems, at least not from an economic point of view (e.g. decreased stocking density) (Larsen et al., 2016).

\section{Husbandry systems and enrichment}

Husbandry systems, especially during rearing period, have been identified as relevant factors concerning the development of abnormal behaviour (Schrøder-Petersen and Simonsen, 2001). Tail lesions, which have been scored during fattening, can refer to several factors of early housing conditions such as for example the number of feeding places per animal or the percentage of floor space covered with slats during the suckling period as well as feeding type and feeding spaces during rearing (Smulders et al., 2008). An enriched environment during weeks four, seven and eighteen lead to less tail biting in comparison to conventional pens (Petersen et al., 1995). Furthermore, a reduction in stress by reduced regrouping, reduced transports, adequate feeding, additional provision of straw and optimal climate decrease the tail biting level (Ekkel et al., 1995). Besides management, the flooring of the husbandry system is also related to the incidence of tail lesions (Schrøder-Petersen and Simonsen, 2001). The risk increases with fully or partly slatted floors in comparison to solid ones (Moinard et al., 2003; Schrøder-Petersen and Simonsen, 2001). Slatted floors are permeable to a higher level of harmful gases (Schrøder-Petersen and Simonsen, 2001; van Putten, 1969); additionally the low level of bedding has a negative effect on the well-being of the animals (EFSA, 2007). 
Although deep, straw bedded systems are not the best way to prevent tail biting either, Hunter et al. (2001) showed that the daily offer of straw keeps the interest of the animals and provides for less biting compared to permanent or no straw provision. Manipulable material as straw or else wood is considered to be very important to enrich the environment and therefore reduce tail biting (EFSA, 2007). Rooting behaviour is a natural need of pigs and if they cannot satisfy it with bedding material, they will look for alternatives, which could be enrichment material or in the worst case the tail of a pen-mate (EFSA, 2014). For this reason, there is also a need for enrichment materials in conventional systems to keep the pigs occupied, although the bedding type is considered to be more important (Valros et al., 2016). The material needs to fulfil a number of requirements in order to be beneficial and not harmful (EFSA, 2014). Therefore, it has to be safe for the pigs, deformable and moveable by pig manipulation, multifunctional and have feed-related properties, such as smell, taste and nutritional value (EFSA, 2014). For hygienic reasons and to ensure that the animals do not lose interest, it should be replaced and renewed regularly. Ursinus et al. (2014b) showed that jute bags reduce tail biting in pigs during rearing. Telkänranta et al. (2014) detected that the use of fresh wood attached to chains was preferred to bare chains and that the incidence of tail biting could be reduced as well. The use of nutritious enrichment materials such as wood shavings or straw is advantageous due to the additional utilisation as bedding material. In addition, the digestive tract of the animals is stimulated if the animals ingest fibrous materials (Schrøder-Petersen and Simonsen, 2001).

\section{Feeding}

Feeding-related issues are very important to avoid tail biting (Valros et al., 2016). The structure of the feeding, the components and the feeding method are of significance here. Increased numbers of tail lesions were detected by Hunter et al. (2001) for pigs fed by pelleted diets in comparison to liquid-fed pigs. In contrast, Palander (2016) investigated a higher risk for liquid feeding. The reason for the inconsistent findings is the variability of quality and contents at liquid feeding compared to pellet feeding (Palander, 2016). Various studies have considered energy, protein fibre and minerals within the feeding to be important in relation to tail biting (D'Eath et al., 2014; Schrøder-Petersen and Simonsen, 2001; Valros et al., 2016). However, it has not been possible to prove that certain components have certain consequences (Valros, 2018). Previous findings have shown that a lack of minerals increases the attractiveness of blood (Fraser, 1987), a higher amount of tryptophan is related to reduced biting prevalence (Martinez-Trejo et al., 2009) and Palander (2016) found a negative effect of wheat in a diet of weaners. Nevertheless, the method of feeding appears to be a risk factor as well. Limited space at the feeder leads to increased tail biting as a result of rank fights (Hunter et al., 2001). The competition of pigs who want to eat simultaneously leads to frustration and to the "sudden forceful" type of biting (Taylor et al., 2010). Sutherland et al. (2009) showed that $30 \%$ of tail 
biting occurred at the feeder. In addition, a restriction of the space at the feeding trough led to health-related consequences as a reduced level of essential amino acids in the blood, as well as changes in the intestinal wall structure (Palander et al., 2013). Although the feeding was ad libitum, the lack of space meant that some animals ate less, which caused frustration (Palander et al., 2013). Moinard et al. (2003) detected a correlation of tail biting to pigs per feeder space and animal density in the pen; thus, more space means fewer behavioural abnormities.

\section{Space allowance}

A reduced space allowance, lower than those currently recommended in the European Union, leads to an increase in tail lesions (Krider et al., 1975). With more than $110 \mathrm{~kg} / \mathrm{m}^{2}$ during the rearing period (Moinard et al., 2003) and with less than $0.31 \mathrm{~m}^{2}$ for growing pigs (30 - $40 \mathrm{~kg}$ ) (Goossens et al., 2008) the risk of tail lesions increases significantly. Munsterhjelm et al. (2017) showed a reduction in the prevalence of tail lesions with increasing space from 0.7 to $1.5 \mathrm{~m}^{2}$. Insufficient space can have a negative effects for heavy pig production of fattening pigs until up to $170 \mathrm{~kg}$ in particular (Scollo et al., 2016). In addition, the shape of the pen has an effect on the aggressive behaviour of the animals; it has been shown that rectangular pens reduce aggressive behaviour and increase animal welfare in comparison to square pens (Barnett et al., 1993).

As the space allowance affects the level of animal welfare, the Federal Ministry of Food and Agriculture of Germany has started to develop a national animal welfare label for pigs (BMEL, 2019). For fattening pigs from $50-110 \mathrm{~kg}$, there will be three different levels, concerning, on the one hand, the space allowance of the pigs and, on the other hand, the structure of the housing environment (BMEL, 2019). The legal minimum space requirement for fattening pigs in this weight class $(50-110 \mathrm{~kg})$ is $0.75 \mathrm{~m}$. At the first level, the pigs receive $20 \%$ more space $\left(0.9 \mathrm{~m}^{2}\right)$, at the second level, they receive $47 \%$ more space $\left(1.1 \mathrm{~m}^{2}\right)$ and at the third level they receive twice as much space $\left(1.5 \mathrm{~m}^{2}\right)$, including $0.5 \mathrm{~m}^{2}$ of exercise area (BMEL, 2019). Considering the previous research findings (Munsterhjelm et al., 2017), level three in particular would reduce the risk of tail biting considerably.

\section{Sex}

Castrated pigs tend to be tail bitten more often than females. Especially if the number of females in the pen is higher, the risk of castrate victims increases (Kritas and Morrison, 2004). In female, single-sex pens, the number of biting pigs is higher than in pens with only males (Zonderland et al., 2010). The increased female interest in the ano-genital area of other pigs due to the beginning of sexual maturity at the end of rearing and the generally higher activity level are reasons for the inclination to more tail biting (Sambraus, 1985; Schrøder-Petersen 
and Simonsen, 2001). Zonderland et al. (2010) found that females tend to bite in the first month after weaning.

\section{Genetics}

Whether a pig could be a biter or a victim is suspected to be influenced by their genetics in many studies, but has not yet been proven (Valros, 2018). Taylor et al. (2010) assume genetic causes, especially for the "obsessive" type of tail biting. Sambraus (1985) suspected that the selection of breeding on certain carcass traits was a reason for more pronounced tail biting. A tendency of certain breeds to bite their tails has also been detected in other studies. Yorkshire pigs have a higher prevalence to suffer tail lesions than Landrace pigs and, in reverse, Landrace pigs are likely to be biters (Breuer et al., 2005; Sinisalo et al., 2012). Duroc pigs are considered as biters compared to the other two breeds. Additionally, they spend more time on manipulable enrichment material. (Breuer et al., 2003). A heritability of tail biting could not be established, even if Breuer et al. (2005) found a significant effect for Landrace. However, the propensity to bite seems to be related to a tendency to grow rapidly and with high leanness (Breuer et al., 2005; Ursinus et al., 2014b). Biters are phenotypically heavier and victims have lower backfat values (Ursinus et al., 2014b). In addition, Sinisalo et al. (2012) found a lower genetic growth potential for pigs with tail lesions. In a selection of indirect genetic growth effects, Camerlink et al. (2015) were able to show less tail biting and a reduction in the manipulation of enrichment material. Besides the correlation to performance traits, there is also a connection to explorative behavioural patterns of potential biters and victims of tail biting (Brunberg et al., 2013). However, Brunberg et al. (2013) found that biters and victims differ less genetically from each other than neutral animals, which might be important in finding a tail-biting-resistant genotype (Valros, 2018).

\section{Acute risk factors}

Acute risk factors are less predictable than chronic factors and include indoor climate, temperature and the health status of the pigs (Valros, 2018). These parameters involve spontaneous changes which cause stress (Weary et al., 2008) and therefore induce tail biting.

The stable climate, which includes stable temperature, good air quality and regular ventilation, may have an effect (Schrøder-Petersen and Simonsen, 2001). Farmers ranked managing air movements, air quality and appropriate temperature in the pen among the top ten factors to avoid tail biting (Valros et al., 2016). Since inducing inadequate climate, as van Putten (1969) did, is not conducted nowadays, findings concerning climate are based on measurements obtained during the research of other parameters. Spontaneous changes in temperature, for example, have been observed as a trigger for tail biting (D'Eath et al., 2014). Poor air quality 
and non-detectable air movements are known to be a reason for tail biting in heavy pigs (Scollo et al., 2016).

The health status of the pigs is also affected by the stable climate (Valros et al., 2016). The number of tail lesions is higher in groups which suffer from respiratory diseases or rectal prolapses (Moinard et al., 2003). Moreover, D'Eath et al. (2014) summarised that vaccinated animals have a lower tail biting risk through reduced risk of emergent diseases.

\section{Prediction of tail biting}

The prediction or early discovery of a tail biting outbreak leads to effective intervention (Valros, 2018). Behaviours which have been found to change before an outbreak occurs exist as increased activity level, increased interactions with enrichment material, and a changed proportion of tail posture (more tails hang down or are caught between the legs) (Larsen et al., 2016). Especially the increased activity level has been discussed as a reliable prediction tool in some studies (Statham et al., 2009; Ursinus et al., 2014a). Furthermore, intensive chewing of enrichment material or parts of the body of the pen-mates has been observed as a typical sign of a pending outbreak (Ursinus et al., 2014a). The well-being of the pig can be concluded from the tail posture. The position of the tail - whether it is curled up or hangs down - can predict also tail biting (Statham et al., 2009; Zonderland et al., 2009). D'Eath et al. (2018) found a method using time-of-flight 3D cameras and machine vision algorithms to detect low tail posture. Increasing low tail posture in the pen can be a sign of an upcoming tail biting outbreak (D'Eath et al., 2018).

Also, feeding behaviour can be an indicator of tail biting. Especially automatic systems, where the visits of the individual animals can be recorded, are suitable for prediction. Low feeding frequencies of the whole pen can predict future tail biting nine weeks before an outbreak (Wallenbeck and Keeling, 2013). In contrast, increased feeding frequencies for individual pigs show potential victims but after tail biting occurred, these victims decreased their amount of feeding (Wallenbeck and Keeling, 2013). There are investigations into the use of cameras in combination with computer vison algorithms to detect pig behaviour (Oczak et al., 2014). The advantages of an automated system are low workload, precise analysis, continuity and noninvasiveness (Rushen et al., 2012). The prediction of the behavioural disorder tail biting by an automatic warning system has not yet been successful but might be possible with further research (Larsen et al., 2016). 


\section{Research factors of the present dissertation}

Tail docking is painful and cannot prevent tail biting, but being bitten is hurtful as well, in addition it can happen repeatedly (Valros, 2018). For undocked pigs, there is a higher risk of being bitten, but there is also the possibility of no painful tail lesions. In contrast, docked pigs suffer from docking pain and might be bitten (Valros and Heinonen, 2015). Alternative farrowing systems such as free farrowing pens or group housing systems offer more comfort for the sow and opportunities for early socialisation for the piglets (D'Eath, 2005). The housing environment during the suckling period affects the behaviour of the pigs in later life as well as docking (Ekkel et al., 1995; Weary et al., 2008). Socialised piglets, which grew up in a loose housing system, are able to cope with stress easier (Oostindjer et al., 2011). Furthermore, the rearing environment influences the behaviour of the pigs (Petersen et al., 1995; SchrøderPetersen and Simonsen, 2001). Consequently, the docking status as well as the farrowing and rearing system has an effect on pigs' behaviour. However, it has not previously been investigated how far undocked and docked animals differ in their tail biting behaviour and whether statistically significant effects of the housing systems during nursery, rearing and fattening period can be detected.

\section{References}

Barnett, J.L., Cronin, G.M., McCallum, T.H., Newman, E.A., 1993. Effects of pen size/shape and design on aggression when grouping unfamiliar adult pigs. Applied Animal Behaviour Science 36 (2-3), 111-122. https://doi.org/10.1016/0168-1591(93)90003-8.

Beattie, V.E., Breuer, K., O'Connell, N.E., Sneddon, I.A., Mercer, J.T., Rance, K.A., Sutcliffe, M.E.M., Edwards, S.A., 2005. Factors identifying pigs predisposed to tail biting. Animal Science 80 (3), 307-312. https://doi.org/10.1079/ASC40040307.

Beattie, V.E., Sneddon, I.A., Walker, N., Weatherup, R.N., 2001. Environmental enrichment of intensive pig housing using spent mushroom compost. Animal Science 72 (1), 35-42. https://doi.org/10.1017/S1357729800055533.

Blackshaw, J.K., 1981. Some behavioural deviations in weaned domestic pigs: persistent inguinal nose thrusting, and tail and ear biting. Animal Science 33 (3), 325-332. https://doi.org/10.1017/S000335610003172X.

BMEL, 2019. Bundesministerium für Ernährung und Landwirtschaft: Das staatliche Tierwohlkennzeichen für Schweine - Mehr Platz und Struktur im Stall. https://www.bmel.de/SharedDocs/Downloads/DE/Broschueren/FlyerPoster/Tierwohlkennzeichen_Schwein_Grafiken.html. 
Bracke, M.B.M., Lauwere, C.C. de, Wind, S.M.M., Zonderland, J.J., 2013. Attitudes of Dutch Pig Farmers Towards Tail Biting and Tail Docking. Journal of Agricultural and Environmental Ethics 26 (4), 847-868. https://doi.org/10.1007/s10806-012-9410-2.

Breuer, K., Sutcliffe, M.E.M., Mercer, J.T., Rance, K.A., Beattie, V.E., Sneddon, I.A., Edwards, S.A., 2003. The effect of breed on the development of adverse social behaviours in pigs. Applied Animal Behaviour Science 84 (1), 59-74. https://doi.org/10.1016/S0168-1591(03)00147-3.

Breuer, K., Sutcliffe, M.E.M., Mercer, J.T., Rance, K.A., O'Connell, N.E., Sneddon, I.A., Edwards, S.A., 2005. Heritability of clinical tail-biting and its relation to performance traits. Livestock Production Science 93 (1), 87-94. https://doi.org/10.1016/j.livprodsci.2004.11.009.

Brunberg, E., Jensen, P., Isaksson, A., Keeling, L.J., 2013. Brain gene expression differences are associated with abnormal tail biting behavior in pigs. Genes, brain, and behavior 12 (2), 275-281. https://doi.org/10.1111/gbb.12002.

Camerlink, I., Ursinus, W.W., Bijma, P., Kemp, B., Bolhuis, J.E., 2015. Indirect genetic effects for growth rate in domestic pigs alter aggressive and manipulative biting behaviour. Behavior genetics 45 (1), 117-126. https://doi.org/10.1007/s10519-014-96719.

Council of European Union, 2008. Council directive 2008/120/EC.

D'Eath, R.B., 2005. Socialising piglets before weaning improves social hierarchy formation when pigs are mixed post-weaning. Applied Animal Behaviour Science 93 (3-4), 199211. https://doi.org/10.1016/j.applanim.2004.11.019.

D'Eath, R.B., Arnott, G., Turner, S.P., Jensen, T., Lahrmann, H.P., Busch, M.E., Niemi, J.K., Lawrence, A.B., Sandøe, P., 2014. Injurious tail biting in pigs: how can it be controlled in existing systems without tail docking? Animal 8 (9), 1479-1497. https://doi.org/10.1017/S1751731114001359.

D'Eath, R.B., Jack, M., Futro, A., Talbot, D., Zhu, Q., Barclay, D., Baxter, E.M., 2018. Automatic early warning of tail biting in pigs: 3D cameras can detect lowered tail posture before an outbreak. PloS one 13 (4), e0194524. https://doi.org/10.1371/journal.pone.0194524.

EFSA, 2007. European Food Safety Authority: The risks associated with tail biting in pigs and possible means to reduce the need for tail docking considering the different housing and husbandry systems - Scientific Opinion of the Panel on Animal Health and Welfare. EFSA Journal 5 (12), 611. https://doi.org/10.2903/j.efsa.2007.611.

EFSA, 2014. European Food Safety Authority: Scientific Opinion concerning a Multifactorial approach on the use of animal and non-animal-based measures to assess the welfare of pigs. EFSA Journal 12 (5). https://doi.org/10.2903/j.efsa.2014.3702. 
Ekkel, E.D., van Doorn, C.E., Hessing, M.J., Tielen, M.J., 1995. The Specific-Stress-Free housing system has positive effects on productivity, health, and welfare of pigs. Journal of Animal Science 73 (6), 1544-1551. https://doi.org/10.2527/1995.7361544x.

Fraser, D., 1987. Attraction to blood as a factor in tail-biting by pigs. Applied Animal Behaviour Science 17 (1-2), 61-68. https://doi.org/10.1016/0168-1591(87)90008-6.

Goossens, X., Sobry, L., Ödberg, F., Tuyttens, F., Maes, D., Smet, S. de, Nevens, F., Opsomer, G., Lommelen, F., Geers, R., 2008. A population-based on-farm evaluation protocol for comparing the welfare of pigs between farms. Animal Welfare 17, 35-41.

Herskin, M.S., Thodberg, K., Jensen, H.E., 2015. Effects of tail docking and docking length on neuroanatomical changes in healed tail tips of pigs. Animal 9 (4), 677-681. https://doi.org/10.1017/S1751731114002857.

Hughes, B.O., Duncan, I.J.H., 1988. The notion of ethological 'need', models of motivation and animal welfare. Animal Behaviour 36 (6), 1696-1707. https://doi.org/10.1016/S00033472(88)80110-6.

Hunter, E.J., Jones, T.A., Guise, H.J., Penny, R.H., Hoste, S., 2001. The relationship between tail biting in pigs, docking procedure and other management practices. Veterinary journal 161 (1), 72-79. https://doi.org/10.1053/tvjl.2000.0520.

Krider, J.L., Albright, J.L., Plumlee, M.P., Conrad, J.H., Sinclair, C.L., Underwood, L., Jones, R.G., Harrington, R.B., 1975. Magnesium Supplementation, Space and Docking Effects on Swine Performance and Behavior1. Journal of Animal Science 40 (6), 1027-1033. https://doi.org/10.2527/jas1975.4061027x.

Kritas, S.K., Morrison, R.B., 2004. An observational study on tail biting in commercial growerfinisher barns. Journal of Swine Health and Production (12), 17-22.

Larsen, M.L.V., Andersen, H.M.-L., Pedersen, L.J., 2016. Can tail damage outbreaks in the pig be predicted by behavioural change? Veterinary journal 209, 50-56. https://doi.org/10.1016/j.tvjl.2015.12.001.

Larsen, M.L.V., Andersen, H.M.-L., Pedersen, L.J., 2018. Which is the most preventive measure against tail damage in finisher pigs: tail docking, straw provision or lowered stocking density? Animal 12 (6), 1260-1267. https://doi.org/10.1017/S175173111700249X.

Martinez-Trejo, G., Ortega-Cerrilla, M.E., Rodarte-Covarrubias, L.F., Herrera-Haro, J.G., 2009. Aggressiveness and productive performance of piglets supplemented with tryptophan. Journal of Animal and Veterinary Advances 8 (4), 608-611.

Moinard, C., Mendl, M., Nicol, C.J., Green, L.E., 2003. A case control study of on-farm risk factors for tail biting in pigs. Applied Animal Behaviour Science (81 (4)), 333-355. https://doi.org/10.1016/S0168-1591(02)00276-9. 
Munsterhjelm, C., Nordgreen, J., Aae, F., Heinonen, M., Olstad, K., Aasmundstad, T., Janczak, A.M., Valros, A., 2017. To be blamed or pitied? The effect of illness on social behavior, cytokine levels and feed intake in undocked boars. Physiology \& behavior 179 , 298-307. https://doi.org/10.1016/j.physbeh.2017.06.024.

Nannoni, E., Valsami, T., Sardi, L., Martelli, G., 2014. Tail Docking in Pigs: A Review on its Short- And Long-Term Consequences and Effectiveness in Preventing Tail Biting. Italian Journal of Animal Science 13 (1), 3095. https://doi.org/10.4081/ijas.2014.3095.

Naya, A., Traulsen, I., Gertz, M., Hasler, M., Burfeind, O., große Beilage, E., Krieter, J., 2018. Is tail biting in growing pigs reduced by a prolonged suckling period? Applied Animal Behaviour Science. https://doi.org/10.1016/j.applanim.2018.10.020.

Oczak, M., Viazzi, S., Ismayilova, G., Sonoda, L.T., Roulston, N., Fels, M., Bahr, C., Hartung, J., Guarino, M., Berckmans, D., Vranken, E., 2014. Classification of aggressive behaviour in pigs by activity index and multilayer feed forward neural network. Biosystems Engineering 119, 89-97. https://doi.org/10.1016/j.biosystemseng.2014.01.005.

Oostindjer, M., van den Brand, H., Kemp, B., Bolhuis, J.E., 2011. Effects of environmental enrichment and loose housing of lactating sows on piglet behaviour before and after weaning. Applied Animal Behaviour Science 134 (1-2), 31-41. https://doi.org/10.1016/j.applanim.2011.06.011.

Palander, P.A., 2016. The tail biting pig - Nutritional and physiological approaches to understanding the behavior. PhD Thesis. Helsinki, Finland, 88 pp.

Palander, P.A., Heinonen, M., Simpura, I., Edwards, S.A., Valros, A.E., 2013. Jejunal morphology and blood metabolites in tail biting, victim and control pigs. Animal 7 (9), 1523-1531. https://doi.org/10.1017/S1751731113000669.

Petersen, V., Simonsen, H.B., Lawson, L.G., 1995. The effect of environmental stimulation on the development of behaviour in pigs. Applied Animal Behaviour Science 45 (3-4), 215-224. https://doi.org/10.1016/0168-1591(95)00631-2.

Rushen, J., Chapinal, N., am de Passillé, 2012. Automated monitoring of behavioural-based animal welfare indicators. Animal Welfare 21 (3), 339-350. https://doi.org/10.7120/09627286.21.3.339.

Sambraus, H.H., 1985. Mouth-based anomalous syndromes: Ethology of Farm Animals. A Comprehensive Study of the Behavioural Features of Common Farm Animals. World Animal Science A5, 391-422.

Schrøder-Petersen, D.L., Simonsen, H.B., 2001. Tail biting in pigs. Veterinary journal 162 (3), 196-210. https://doi.org/10.1053/tvjl.2001.0605.

Schrøder-Petersen, D.L., Simonsen, H.B., Lawson, L.G., 2003. Tail-in-mouth Behaviour Among Weaner Pigs in Relation to Age, Gender and Group Composition Regarding 
Gender. Acta Agriculturae Scandinavica, Section A — Animal Science 53 (1), 29-34. https://doi.org/10.1080/09064700310002017.

Scollo, A., Contiero, B., Gottardo, F., 2016. Frequency of tail lesions and risk factors for tail biting in heavy pig production from weaning to $170 \mathrm{~kg}$ live weight. Veterinary journal 207, 92-98. https://doi.org/10.1016/j.tvjl.2015.10.056.

Scollo, A., Di Martino, G., Bonfanti, L., Stefani, A.L., Schiavon, E., Marangon, S., Gottardo, F., 2013. Tail docking and the rearing of heavy pigs: the role played by gender and the presence of straw in the control of tail biting. Blood parameters, behaviour and skin lesions. Research in veterinary science 95 (2), 825-830. https://doi.org/10.1016/j.rvsc.2013.06.019.

Sinisalo, A., Niemi, J.K., Heinonen, M., Valros, A., 2012. Tail biting and production performance in fattening pigs. Livestock Science 143 (2-3), 220-225. https://doi.org/10.1016/j.livsci.2011.09.019.

Smulders, D., Hautekiet, V., Verbeke, G., Geerst, R., 2008. Tail and ear biting lesions in pigs: an epidemiological study. Animal Welfare 17, 61-69.

Spoolder, H., Bracke, M., Mueller-Graf, C., Edwards, S., 2011. Preparatory work for the future development of animal based measures for assessing the welfare of pig - Report 2: Preparatory work for the future development of animal based measures for assessing the welfare of weaned, growing and fattening pigs including aspects related to space allowance, floor types, tail biting and need for tail docking. EFS3 8 (7). https://doi.org/10.2903/sp.efsa.2011.EN-181.

Statham, P., Green, L., Bichard, M., Mendl, M., 2009. Predicting tail-biting from behaviour of pigs prior to outbreaks. Applied Animal Behaviour Science 121 (3-4), 157-164. https://doi.org/10.1016/j.applanim.2009.09.011.

Stolba, A., Wood-Gush, D.G.M., 1980. Arousal and exploration in growing pigs in different environments. Applied Animal Ethology 6 (4), 382-383. https://doi.org/10.1016/03043762(80)90140-6.

Studnitz, M., Jensen, M.B., Pedersen, L.J., 2007. Why do pigs root and in what will they root? Applied Animal Behaviour Science 107 (3-4), 183-197. https://doi.org/10.1016/j.applanim.2006.11.013.

Sutherland, M., Bryer, P. J., Krebs, N., McGlone, J. J., 2009. The effect of method of tail docking on tail-biting behaviour and welfare of pigs. Animal Welfare 18 (4).

Sutherland, M.A., 2015. Welfare implications of invasive piglet husbandry procedures, methods of alleviation and alternatives: a review. New Zealand veterinary journal 63 (1), 52-57. https://doi.org/10.1080/00480169.2014.961990.

Taylor, N.R., Main, D.C.J., Mendl, M., Edwards, S.A., 2010. Tail-biting: A new perspective. Veterinary journal 186 (2), 137-147. https://doi.org/10.1016/j.tvjl.2009.08.028. 
Taylor, N.R., Parker, R.M.A., Mendl, M., Edwards, S.A., Main, D.C.J., 2012. Prevalence of risk factors for tail biting on commercial farms and intervention strategies. Veterinary journal 194 (1), 77-83. https://doi.org/10.1016/j.tvjl.2012.03.004.

Telkänranta, H., Bracke, M.B.M., Valros, A., 2014. Fresh wood reduces tail and ear biting and increases exploratory behaviour in finishing pigs. Applied Animal Behaviour Science 161, 51-59. https://doi.org/10.1016/j.applanim.2014.09.007.

Ursinus, W.W., van Reenen, C.G., Kemp, B., Bolhuis, J.E., 2014a. Tail biting behaviour and tail damage in pigs and the relationship with general behaviour: Predicting the inevitable? Applied Animal Behaviour Science 156, 22-36. https://doi.org/10.1016/j.applanim.2014.04.001.

Ursinus, W.W., Wijnen, H.J., Bartels, A.C., Dijvesteijn, N., van Reenen, C.G., Bolhuis, J.E., 2014b. Damaging biting behaviors in intensively kept rearing gilts: the effect of jute sacks and relations with production characteristics. Journal of Animal Science 92 (11), 51935202. https://doi.org/10.2527/jas.2014-7918.

Valros, A., 2018. Tail biting, in: Špinka, M. (Ed.), Advances in pig welfare. Woodhead Publishing an imprint of Elsevier, Duxford, United Kingdom, 137-166.

Valros, A., Ahlström, S., Rintala, H., Häkkinen, T., Saloniemi, H., 2004. The prevalence of tail damage in slaughter pigs in Finland and associations to carcass condemnations. Acta Agriculturae Scandinavica, Section A - Animal Science 54 (4), 213-219. https://doi.org/10.1080/09064700510009234.

Valros, A., Heinonen, M., 2015. Save the pig tail. Porcine health management 1, 2. https://doi.org/10.1186/2055-5660-1-2.

Valros, A., Munsterhjelm, C., Hänninen, L., Kauppinen, T., Heinonen, M., 2016. Managing undocked pigs - on-farm prevention of tail biting and attitudes towards tail biting and docking. Porcine health management 2, 2. https://doi.org/10.1186/s40813-016-0020-7.

van Putten, G., 1969. An Investigation into Tail-Biting among Fattening Pigs. British Veterinary Journal 125 (10), 511-517. https://doi.org/10.1016/S0007-1935(17)48710-0.

Veit, C., 2016. Influence of raw material and weaning management on the occurrence of tailbiting in undocked pigs. PhD Thesis. Hannover.

Walker, P.K., Bilkei, G., 2006. Tail-biting in outdoor pig production. Veterinary journal 171 (2), 367-369. https://doi.org/10.1016/j.tvjl.2004.10.011.

Wallenbeck, A., Keeling, L.J., 2013. Using data from electronic feeders on visit frequency and feed consumption to indicate tail biting outbreaks in commercial pig production. Journal of Animal Science 91 (6), 2879-2884. https://doi.org/10.2527/jas.2012-5848.

Weary, D.M., Jasper, J., Hötzel, M., 2008. Understanding weaning distress. Applied Animal Behaviour Science 110 (1-2), 24-41. https://doi.org/10.1016/j.applanim.2007.03.025. 
Zonderland, J.J., Bracke, M.B.M., den Hartog, L.A., Kemp, B., Spoolder, H.A.M., 2010. Gender effects on tail damage development in single- or mixed-sex groups of weaned piglets. Livestock Science 129 (1-3), 151-158.

https://doi.org/10.1016/j.livsci.2010.01.018.

Zonderland, J.J., Kemp, B., Bracke, M.B.M., den Hartog, L.A., Spoolder, H.A.M., 2011a. Individual piglets' contribution to the development of tail biting. Animal 5 (4), 601-607. https://doi.org/10.1017/S1751731110002132.

Zonderland, J.J., Schepers, F., Bracke, M.B.M., den Hartog, L.A., Kemp, B., Spoolder, H.A.M., 2011b. Characteristics of biter and victim piglets apparent before a tail-biting outbreak. Animal 5 (5), 767-775. https://doi.org/10.1017/S1751731110002326.

Zonderland, J.J., van Riel, J.W., Bracke, M.B.M., Kemp, B., den Hartog, L.A., Spoolder, H.A.M., 2009. Tail posture predicts tail damage among weaned piglets. Applied Animal Behaviour Science 121 (3-4), 165-170. https://doi.org/10.1016/j.applanim.2009.09.002.

Zonderland, J.J., Wolthuis-Fillerup, M., van Reenen, C.G., Bracke, M.B.M., Kemp, B., Hartog, L.A.d., Spoolder, H.A.M., 2008. Prevention and treatment of tail biting in weaned piglets. Applied Animal Behaviour Science 110 (3-4), 269-281.

https://doi.org/10.1016/j.applanim.2007.04.005. 


\section{PAPER ONE}

\section{Tail lesions and losses of docked and undocked pigs in different farrowing and rearing systems}

\section{Maria Gentz ${ }^{1, *}$, Anita Lange ${ }^{1}$, Sebastian Zeidler ${ }^{2}$, Christian Lambertz ${ }^{3, \dagger}$, Matthias Gauly ${ }^{3}$, Onno Burfeind ${ }^{4}$ and Imke Traulsen ${ }^{1}$}

1 Department of Animal Sciences, Livestock Systems, Georg-August-University, Albrecht-Thaer-Weg 3, 37075 Göttingen, Germany. anita.lange@agr.unigoettingen.de (A.L.); imke.traulsen@uni-goettingen.de (I.T.)

2 Department of Animal Sciences, Breeding Informatics, Georg-August-University, Margarethe von Wrangell-Weg 7, 37075 Göttingen, Germany. sebastian.zeidler@uni-goettingen.de (S.Z.)

${ }^{3}$ Faculty of Science and Technology, Free University of Bolzano, Universitätsplatz 5, 39100 Bolzano, Italy. christian.lambertz@fibl.org (C.L.); matthias.gauly@unibz.it (M.Ga.)

${ }^{4}$ Chamber of Agriculture of Schleswig-Holstein, Gutshof 1, 24327 Blekendorf, Germany. oburfeind@lksh.de (O.B.)

† Research Institute of Organic Agriculture (FiBL), Kasseler Straße 1a, 60486 Frankfurt am Main, Germany.

* Correspondence: maria.gentz@uni-goettingen.de; Tel: +495513925774 


\section{Abstract}

This study aimed to investigate the effects of farrowing and rearing systems on tail lesions and losses of docked and undocked pigs. Pigs from three farrowing systems: Conventional farrowing crate (FC), free farrowing (FF) and group housing of lactating sows (GH) were randomly allocated to different rearing systems: A conventional system (CONV), where the pigs were regrouped and transferred to conventional finishing pens at ten weeks of age or a wean-to-finish (W-F) system, where the pigs remained in their pens until slaughter with higher space allowance during rearing. Weekly, tail lesions and losses were assessed individually. The incidence of tail lesions was higher in undocked CONV pigs compared to undocked W-F pigs (maximum: CONV 58.01\%, W-F 41.16\%). The rearing system had a significant effect on tail losses at the end of finishing (CONV 67.63\%, W-F 38.2\%). The significant effect of the rearing system might be explained by higher space allowance during rearing and reduced regrouping stress for W-F pigs. In conclusion, farrowing systems showed no effects, but the W-F rearing system reduces the frequency of tail lesions and losses; the curves of tail lesions increased slower and stayed on a lower level, which resulted in lower losses as well.

Keywords: tail lesions; tail losses; farrowing system; rearing system; undocked pigs; docked pigs; rearing; fattening; scoring; assessment

\section{Introduction}

One of the most important challenges in modern pig production is tail biting (SchrøderPetersen and Simonsen, 2001). It occurs in a wide range of housing systems (Valros, 2018). Taylor et al. (2010) describe three different types of tail biting behaviour, whereby the first type can be divided into two phases: "Pre-damage" and "damage". "Pre-damage" describes a gentle and harmless chewing on the tail of another pig. The tail remains complete and does not show any lesions (Schrøder-Petersen and Simonsen, 2001). "Damage" results in a bleeding tail caused by dental manipulation (biting) (Taylor et al., 2010). This type could be caused by a lack of enrichment material (Beattie et al., 1996). The second type "sudden-forceful" biting is defined by grabs and yanks of the tail including partial or full loss of the tail (Taylor et al., 2010; van Putten, 1969). This type is often caused by a lack of resources such as water or feed (Moinard et al., 2003). The third type "obsessive" tail biting is defined by repeated grabs and yanks of the tail including partial or full loss of the tail (Taylor et al., 2010). The triggering factor for this type of biting is unknown, but it may be caused by a possible genetically induced aggressiveness or the increased attraction to blood (Taylor et al., 2010). To prevent all three types of biting, the pigs which tail bite could be removed from the pen. In any case the bitten pigs need to be treated and, if necessary, also removed from the pen. The first type of tail 
biting might be reduced by providing suitable objects for manipulation, chewing and rooting (Taylor et al., 2010; Zonderland et al., 2008).

Tail biting is an issue which is caused by many factors. Pigs suffering from stress may react with this abnormal behaviour (Weary et al., 2008). Included among the multiple risk factors that initiate stressful situations for the pigs are climatic and light conditions (Parker et al., 2010), sex (Zonderland et al., 2010), husbandry environment (Cox and Cooper, 2001), feeding (Statham et al., 2011) and group size (Meyer-Hamme et al., 2016). Climate and light conditions can cause discomfort for pigs if there is no or too strong ventilation, such as a draught, as well as poor air quality or seasonal effects of cold or heat stress (Parker et al., 2010; SchrøderPetersen and Simonsen, 2001; Valros, 2018). Many studies state sex as an influencing factor (Schrøder-Petersen and Simonsen, 2001; Valros, 2018; Zonderland et al., 2010). Studies as well as farmers often detect small female animals to be the biters (Zonderland et al., 2010). This may be due to the higher level of activity attributed to them, combined with their higher propensity to the ano-genital area of other pigs during the first month after weaning (SchrøderPetersen and Simonsen, 2001; Zonderland et al., 2010). Pigs try to compensate for the stressful influences that affect them by different coping strategies. This might result in manipulation of the environment or in the biting of other pigs (Benus et al., 1991; Rushen, 1993).

Consequently, a reduction of stressful factors and an optimization of housing conditions to increase animal welfare is a way to reduce tail biting. For example, early socialization could help the pigs to handle stressful situations better (D'Eath, 2005). Socializing piglets early in life by mixing litters prior to weaning has long-term benefits for the social behaviour (D'Eath, 2005). After weaning, the animals form a new hierarchy more quickly. Furthermore, reduced stress levels and fewer lesions after fights were observed (D'Eath, 2005). Thus, farrowing systems which allow an early litter mixing are advantageous.

Only free farrowing systems fully allow an early socialisation with the sow and also improve the welfare of the mother (Oostindjer et al., 2011). Freely moving sows can act more naturally and this is enabling the piglets to be more explorative and less aggressive (Cox and Cooper, 2001). Further, the environment during lactation as well as additional enrichment materials positively affect the ability to adapt to a new rearing environment (Oostindjer et al., 2011). Group housing of sows and their litters during the suckling period is reduces agonistic interactions after regrouping, and the pigs have fewer skin lesions (Bohnenkamp et al., 2013).

Especially in North America, the wean-to-finish rearing system (W-F) is used (Davis et al., 2006; Wolter et al., 2001). This means that the pigs are only moved to a new pen at weaning and then remain in the pens until the end of the fattening period (Wolter et al., 2002). In this system, pigs generally have twice the space allowance during the rearing period compared to 
conventional systems (Davis et al., 2006). The W-F is designed to achieve lower rearing losses, reduced animal transport and performance enhancement, as well as reduced working time for cleaning and disinfection (Connor, 1998; Davis et al., 2006). In addition, stress was lowered by reducing the number of regroupings (Coutellier et al., 2007). The main problem of this housing system is the poor use of space during rearing. The fact that W-F pigs are housed in the same pen after weaning, until slaughter also means that there are difficulties with the floor construction. Currently the EU regulations for weaning and fattening pigs are difficult to combine, because the animals need different designs of slatted floors (Council of European Union, 2008).

Tail docking was used to reduce tail biting for many years (Lahrmann et al., 2017; SchrøderPetersen and Simonsen, 2001). However, docking does not prevent tail biting completely and is highly discussable from the ethical point of view (Moinard et al., 2003; Schrøder-Petersen and Simonsen, 2001). Depending on the remaining tails' length, a hypersensitivity of the nerves can initiate bitten pigs to escape (Moinard et al., 2003; Schrøder-Petersen and Simonsen, 2001). It is controversial that tail docking also brings disadvantages like infections or provokes abnormal behaviour towards other regions of the body (Schrøder-Petersen and Simonsen, 2001).

The aim of the current study was to investigate the influence of the farrowing and rearing systems on tail lesions and losses during the rearing and fattening periods under conventional conditions, including effects of the time of socialization, contact to foreign litters, tail docking status, regrouping and rehousing.

\section{Materials and Methods}

\subsection{Animals and Housing}

Data were acquired at the research farm Futterkamp of the Chamber of Agriculture of Schleswig-Holstein between May 2016 and August 2018. During seven batches, 2271 rearing pigs (50\% docked and 50\% undocked, cross-breeds of (Large White $\times$ Landrace) and (Pietrain $\times($ Large White $\times$ Landrace $)$ ) were studied. Data were also collected during the fattening period from a total of 1228 animals randomly selected from the rearing pigs. Males were reared as intact males. Pigs were raised in three farrowing systems: Conventional farrowing crate (FC), a free farrowing system (FF) and a group housing system of lactating sows $(\mathrm{GH})$ (Figure 1) (Gentz et al., 2019). 


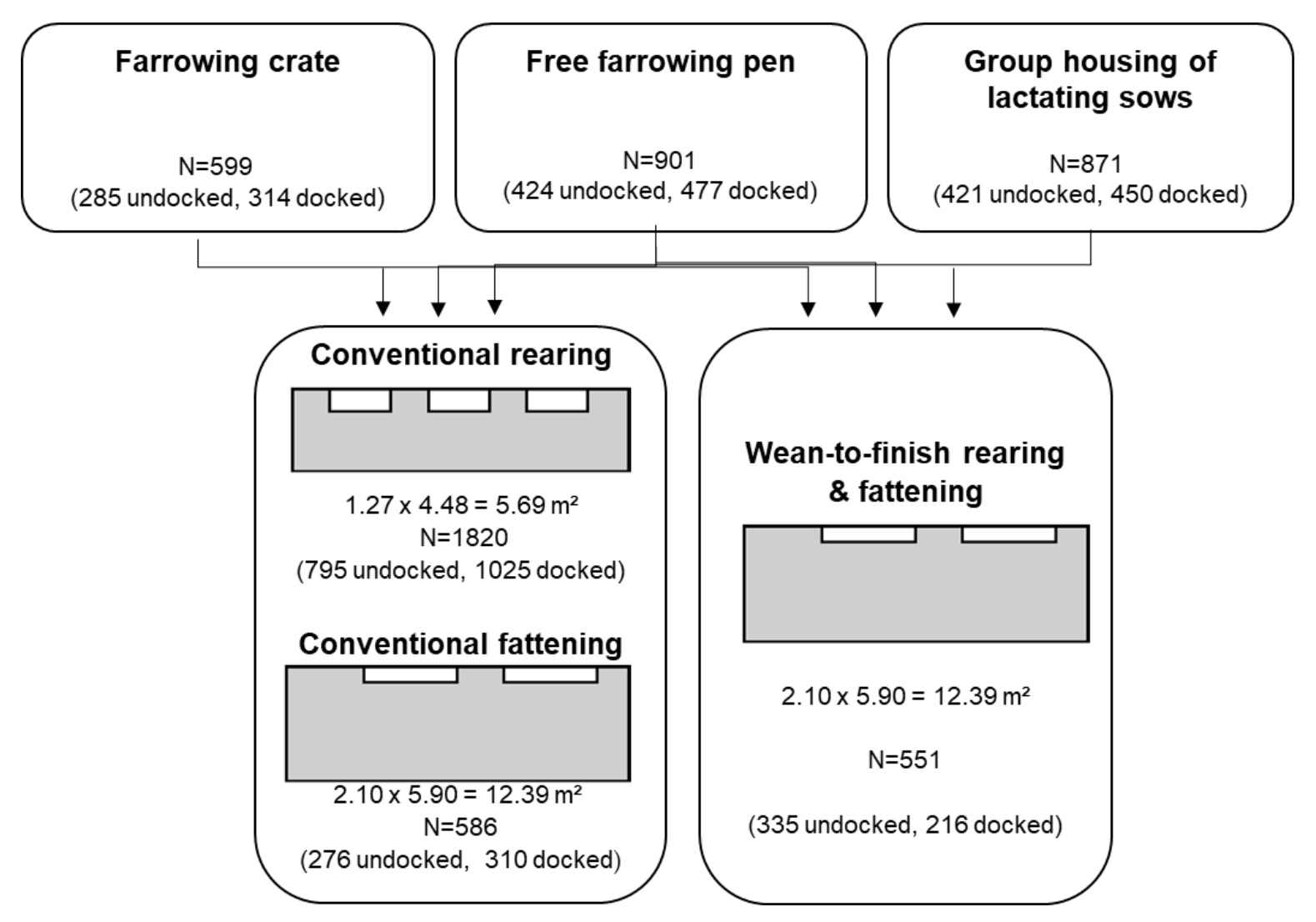

Figure 1. Experimental design $(3 \times 2 \times 2)$ (3 housing systems during suckling, 2 housing systems during rearing and fattening, $50 \%$ undocked and $50 \%$ docked pigs): Overview of the indoor housing systems ( $\mathrm{N}=$ number of pigs) (Gentz et al., 2019).

The sows in the farrowing crates were single housed and their piglets had a space allowance of $5.2 \mathrm{~m}^{2}$. For the FF and the GH animals, two sizes of farrowing pens were used for each system. This is because after two batches of the experiment, a reconstruction of the piglet nest took place. The aim was to reduce the piglet crushes, so that changes in position and architecture of the piglet nest were conducted (Grimberg-Henrici et al., 2019; Lohmeier et al., 2020). The sows in the free farrowing system were single housed as well. Each FF pen had $7.0 \mathrm{~m}^{2} / 7.56 \mathrm{~m}^{2}$ individual space depending on the design of the piglet nest, and sows could move freely except for one batch. In this batch, the sows were fixed for three days postpartum to minimise the crushing losses of piglets. In a GH system, ten sows were housed together. They were single housed in a free-farrowing pen $\left(6.09 \mathrm{~m}^{2} / 5.1 \mathrm{~m}^{2}\right)$. All pens had an entrance for the sows and a separated entrance for the pigs to a joined running area of $30 \mathrm{~m}^{2} / 27.5 \mathrm{~m}^{2}$ which was offered five days postpartum for all animals.

The pigs were weaned 27 days postpartum and divided randomly within farrowing system to two types of rearing systems: Conventional rearing system (CONV rearing, $0.44 \mathrm{~m}^{2} / \mathrm{pig}$ ) and wean-to-finish system (W-F, $0.89 \mathrm{~m}^{2} / \mathrm{pig}$ ) (Figure 1). The W-F pigs were grouped single-sex and the CONV rearing were grouped mixed-sex. Pigs in the CONV rearing group were regrouped and rehoused for finishing and sorted by sex after 40 days of rearing (CONV fattening), while the W-F pigs were raised without regrouping and rehousing until slaughter 
$(\approx 120 \mathrm{~kg})$. The CONV fattening pigs had $0.89 \mathrm{~m}^{2}$ per pig and their pens were identical in structure to those of the W-F pens. In CONV rearing, the pigs were housed in groups of 13 pigs on fully slatted plastic floors. In W-F, the pigs were housed in groups of 14 pigs on fully slatted concrete floors. Both systems were in the same stable. The slat width on CONV fattening and $\mathrm{W}-\mathrm{F}$ pens $(13 \mathrm{~mm}$, tread area $=67 \mathrm{~mm})$ was determined in a special permit (V242-226720/2015(8-1/16)) to allow the pigs to be kept in the same pens from weaning to the end of fattening. In summary, the study was carried out in a 3 farrowing systems $\times 2$ rearing systems $\times 2$ docking status design.

All pigs were fed ad libitum with conventional dry feed adjusted to their age. The feeding system started every day at 06:30 h. During the first week of rearing, the feed, which was also offered during suckling period (14.4 MJ ME/kg, 17.6\% protein, $1.43 \%$ lysine, $0.24 \%$ sodium), was blended with the feed of the rearing period (13.4 MJ ME/kg, 17.0\% protein, $1.30 \%$ lysine, $0.26 \%$ sodium) and fed until the end of rearing. At the beginning of fattening, after another blending of ten days, the pigs were fed a pre-fattening diet (13.4 MJ ME/kg, 16.5\% protein, $1.16 \%$ lysine, $0.20 \%$ sodium) until day 100 of life. At the end of fattening, the feed was blended again and the pigs received end-fattening diet (13.2 MJ ME/kg, 15.5\% protein, $1.01 \%$ lysine, $0.20 \%$ sodium) until slaughter.

All drinking systems consisted of nipples and bowls. The barn temperature during rearing and fattening was automatically regulated by forced ventilation. Artificial lighting was provided between 6:00 $\mathrm{h}$ and 18:00 $\mathrm{h}$. The provision of the enrichment materials was defined (all treatment groups were managed following the same procedure): During the rearing period, the pigs received wood, ropes and troughs filled with peas and grass pellets. During the fattening period, they received wood and ropes. If a tail biting outbreak occurred, they got jute bags and renewed ropes and wood.

\subsection{Data Collection}

Starting one day after weaning, the pigs' tails were scored individually once a week until week 18 of life using a modified Schwarzenauer key, described in (Abriel and Jais, 2013b). A tail "lesion" means that the tail has slight scratches or bite marks (score 1), a "severe lesion" shows deeper, flat lesions (score 2) and a "very severe lesion" is a deep, flat lesion which is greater than $2 \mathrm{~cm}$ (score 3). For the statistical analysis, the tail lesion score was summarised due to the low occurrence of severe and very severe lesions (< $2 \%)$ into "no lesions" (0) and "lesions" (1) (1 summarises score 1, 2, 3).

For the scores of tail losses, the tail was divided into quarters and then the percental loss was assessed which resulted in score 0 (intact tail) to score 4 (100\% lost). For the statistical 
analysis, these scores were also summarised into "intact tail" and "tail loss" due to low occurrence of losses of more than $25 \%$.

\subsection{Statistical Analysis}

The data analysis was conducted with SAS® 9.4 (SAS R Institute Inc, 2008). Due to the nonnormally distribution of the data, the tail lesions and the tail losses were analysed with the GLIMMIX procedure. For each parameter, the model with the lowest values for 'Akaike's information criterion corrected' (AICC) (Hurvich and Tsai, 1989) and 'Bayesian information criterion' (BIC) (Schwarz, 1978) was used for further evaluation.

The model for the prevalence of tail lesions included the fixed effects rearing system (CONV, W-F), farrowing system (FC, FF, GH), docking status (undocked, docked), sex (male, female), batch (1-7), assessment week (1-18) and the interaction of assessment week ${ }^{*}$ rearing system*docking status. In this study, we assumed that neither the reconstruction of the piglet nests nor the fixation of the FF sows affected the tail lesions and losses of the pigs systematically in their later life. The significance of differences in the least square means was adapted by the Bonferroni-correction to adjust for multiple comparisons. In addition, the pen was added as a random effect for consideration of the group effect.

The tail loss of the pigs was calculated in two models for the end of the rearing period (week 6 ) and the end of the fattening period (week 18). Both models were only calculated with undocked pigs due to the fact that docked pigs had only very few tail losses $(<1 \%)$. The fixed effects rearing system (CONV, W-F), farrowing system (FC, FF, GH), sex (male, female) were included. The significance of differences in the least square means was adapted by the Bonferroni-correction to adjust for multiple comparisons. In addition, the pen was added as a random effect for consideration of the group effect.

Due to the study design and management routines it was not possible to house undocked CONV and W-F simultaneously during the fattening period. Therefore, the batch effect for the loss model was confounded with the rearing system and could not be considered separately.

\section{Results}

\subsection{Tail Lesions}

A significant effect $(p<0.01)$ of the rearing system, the docking status, the batch, the assessment week and the interaction of assessment week*rearing system*docking status on the prevalence of tail lesions was observed, while the effect of the farrowing system and sex was not significant $(p>0.05)$. The interaction of assessment week*rearing system*docking 
status is shown in Figure 2 and the significant differences within the assessment weeks are shown in Table 1.

After the second assessment week, the percentage of the pigs of all treatment groups with tail lesions increased (Figure 2). The percentage of the undocked CONV pigs increased through the whole rearing period until they reach their maximum in week 7 (58.0\%). After this peak, the curve took a jagged course with decreasing tendency during the fattening period. At almost all times, the prevalence of tail lesions for undocked pigs was significantly higher than for docked pigs (Table 1). The percentage of the undocked W-F pigs with tail lesions increased during the rearing period as well. This treatment group reached their maximum later, on a lower level, in week 11 (41.2\%), thereafter the graph shows a decreasing tendency. From week 12 onwards, the undocked W-F animals showed no significant differences in comparison to the two docked treatment groups (Table 1). In contrast to the other groups, the docked CONV pigs reached their maximum of pigs with tail lesions at the end of the rearing period (week 6, 20.0\%). The maximum prevalence of the peak of the docked W-F was only one third of the size of the undocked CONV.

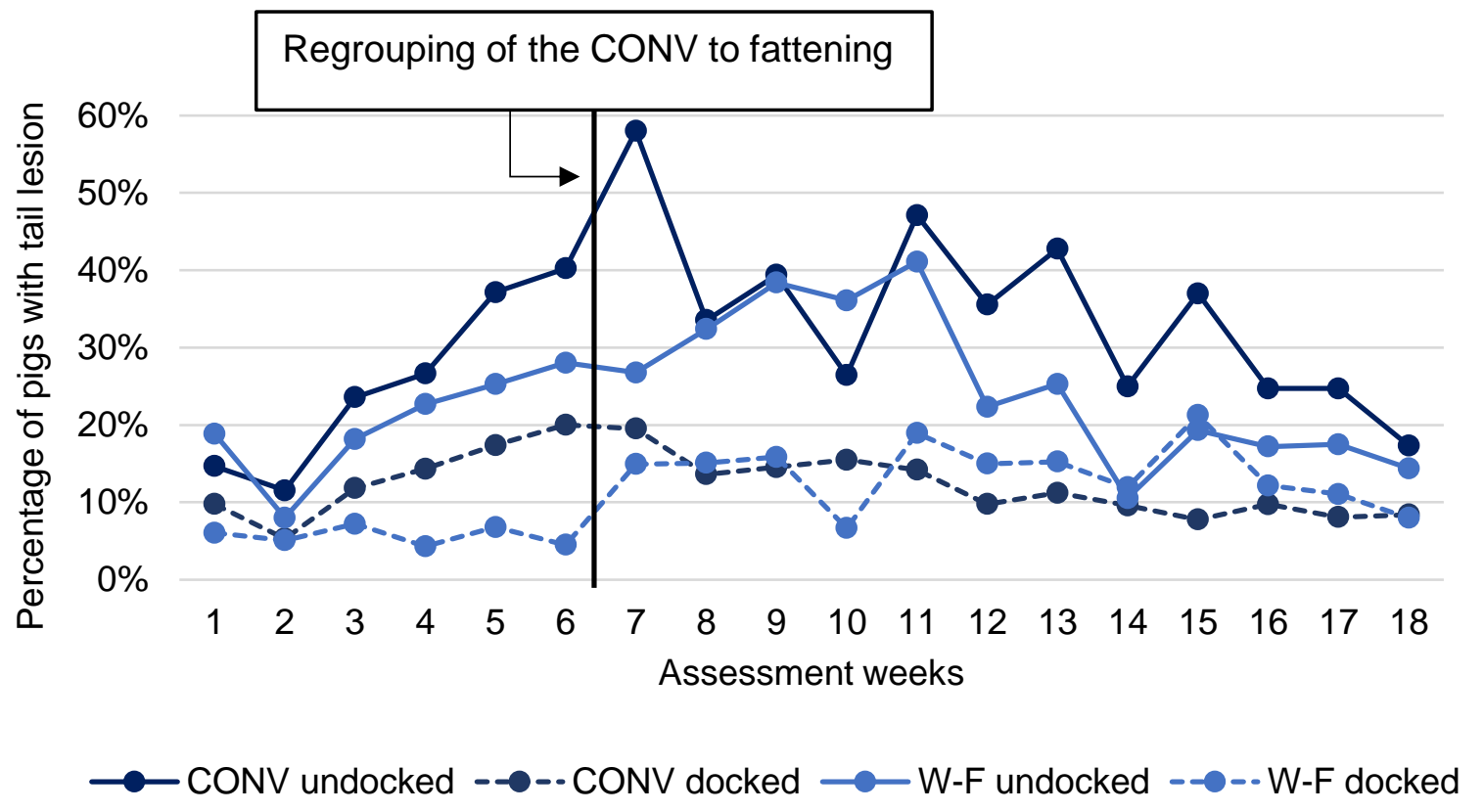

Figure 2. Frequency of back transformed tail lesion estimates during of the rearing and fattening periods for docked and undocked animals per rearing system (CONV=conventional rearing system; $\mathrm{W}-\mathrm{F}=$ Wean-to-Finish rearing system). 
Table 1. Multiple comparison of the Least Square-means differences of the interaction assessment week ${ }^{*}$ rearing system*docking status by Bonferroni within the assessment week $(p<0.05)$.

\begin{tabular}{ccccccccccccccccccc}
\hline Assessment week & 1 & 2 & 3 & 4 & 5 & 6 & 7 & 8 & 9 & 10 & 11 & 12 & 13 & 14 & 15 & 16 & 17 & 18 \\
\hline CONV undocked & $a$ & $a$ & $a$ & $a$ & $a$ & $a$ & $a$ & $a$ & $a$ & $a b$ & $a$ & $a$ & $a$ & $a$ & $a$ & $a$ & $a$ & $a$ \\
CONV docked & $a$ & $b$ & $b$ & $b$ & $b$ & $b$ & $b$ & $b$ & $b$ & $b$ & $b$ & $b$ & $b$ & $b$ & $b$ & $b$ & $b$ & $a$ \\
W-F undocked & $a$ & $a b$ & $a b$ & $a$ & $a b$ & $a b$ & $b$ & $a$ & $a$ & $a c$ & $a$ & $a b$ & $a b$ & $a b$ & $a b$ & $a b$ & $a b$ & $a$ \\
W-F docked & $a$ & $a b$ & $b$ & $b$ & $b$ & $c$ & $b$ & $a b$ & $b$ & $b$ & $b$ & $b$ & $b$ & $a b$ & $a$ & $a b$ & $a b$ & $a$
\end{tabular}

a-c: Significant differences of docking status*rearing system interaction within weeks $(p<0.05)$.

The back transformed Least Square means of the batches showed a decreasing tendency over time (Figure 3). Batches 1 and 2 were nearly on the same level of tail lesions $(30.0 \%)$, whereas batches 3 to 7 ranged between 19.1\% and 9.5\% tail lesions. Batch 6 showed the lowest values $(9.5 \%)$.

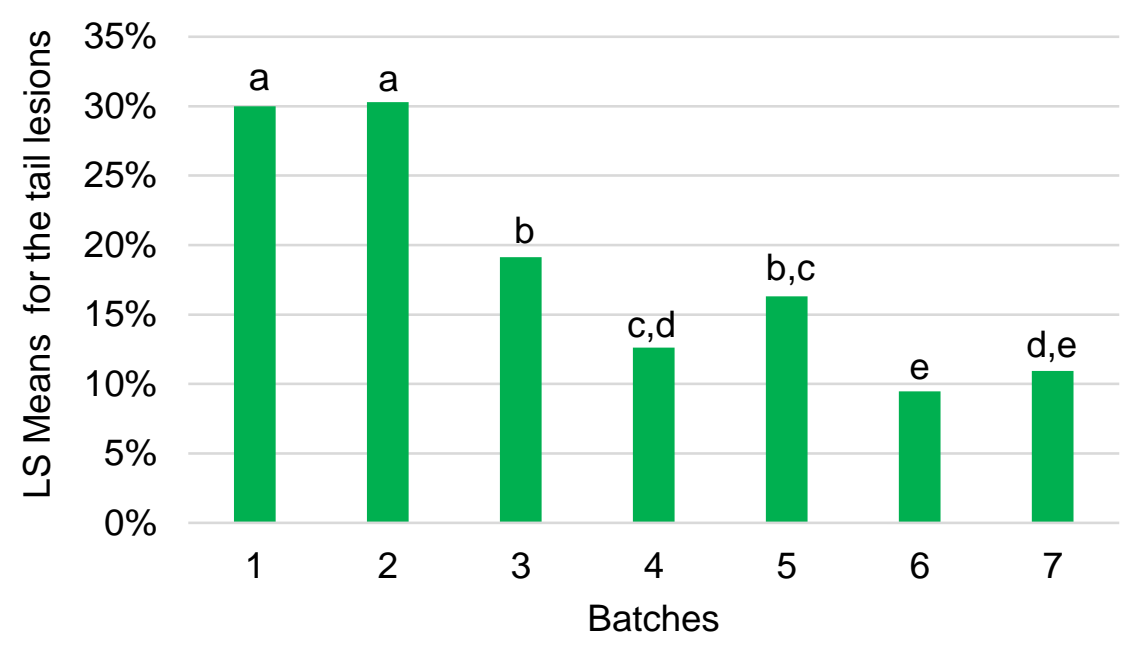

Figure 3. LS-means of back transformed tail lesion estimates of the batches. a-e: Significant differences between the batches $(p<0.05)$.

The farrowing systems only showed non-significant, numerical differences $(p>0.05 ; \mathrm{GH}=$ $16.6 \%, \mathrm{FF}=17.4 \%, \mathrm{FC}=17.4 \%)$.

\subsection{Tail Losses}

The models of the tail losses for the end of rearing and the end of fattening periods showed comparable results. A significant effect $(p<0.05)$ of the rearing system was observed for tail losses. The effects of the farrowing system and the sex were not significant $(p>0.05)$.

At the end of the rearing period, $16.3 \%$ of the undocked CONV pigs showed tail losses (Figure 4). Only $2.9 \%$ of the W-F animals showed tail losses at the end of rearing, less than one fifth 
compared to the CONV animals. At the end of fattening period most of the CONV pigs lost parts of their tails (67.6\%). Only $38.2 \%$ of the W-F animals lost parts of their tails. Nevertheless, the differences between CONV and W-F are significant at the end of rearing and fattening periods.

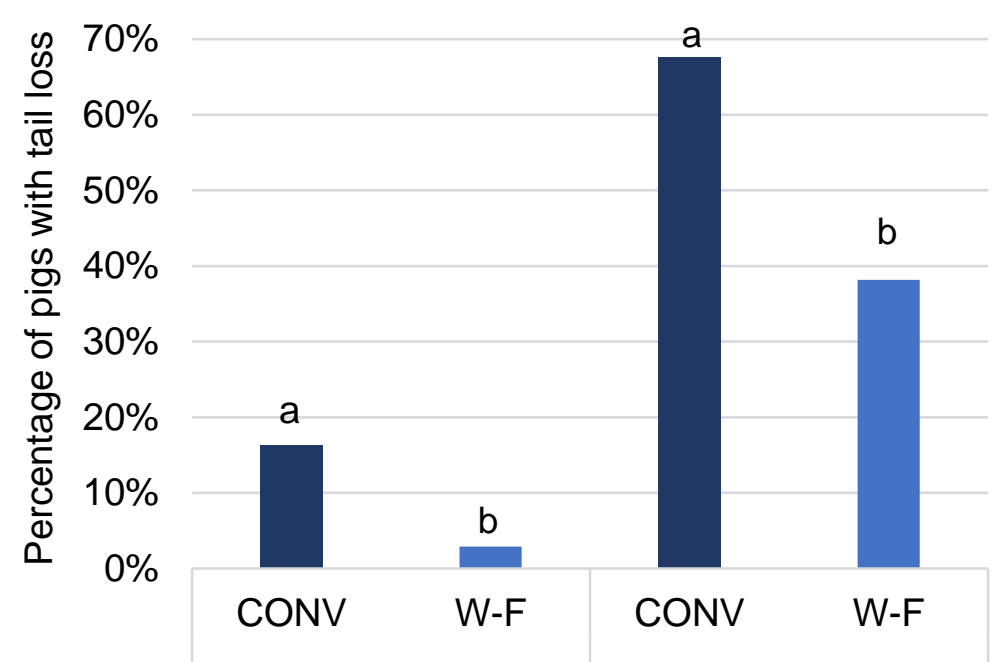

End of rearing period End of fattening period

Figure 4. LS-means of back transformed tail loss estimates of the rearing systems of undocked pigs for end of rearing and end of fattening periods.

$\mathrm{a}, \mathrm{b}$ : Significant differences between the rearing systems within period $(p<0.05)$.

The male and female pigs showed non-significant, numerical differences ((Figure 5). At the end of the rearing period, $9.8 \%$ of the males showed tail losses (females $=5.1 \%$ ). At the end of the fattening period, $64.9 \%$ of the males lost parts of their tails (females $41.2 \%$ ).

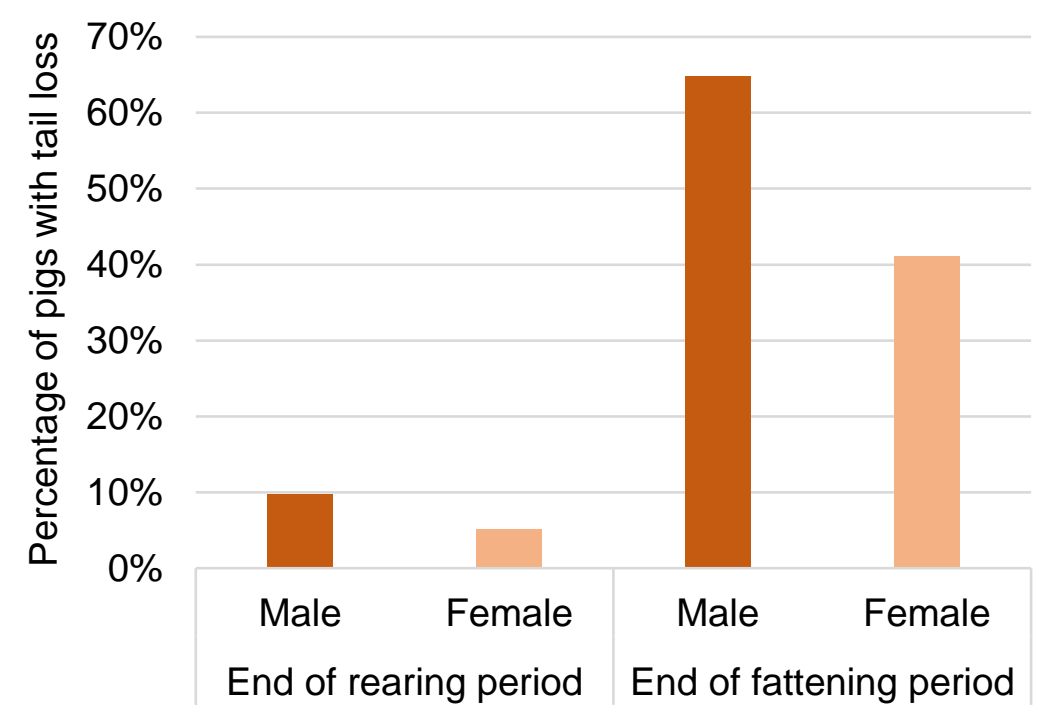

Figure 5. LS-means of back transformed tail loss estimates of the sexes of undocked pigs for end of rearing and end of fattening periods.

The differences of the farrowing systems were numerical (end of rearing period: $F F=5.8 \%$, $\mathrm{FC}=7.5 \%, \mathrm{GH}=8.2 \%$; end of fattening period: $\mathrm{FF}=51.7 \%, \mathrm{FC}=54.1 \%, \mathrm{GH}=53.7$ ). 


\section{Discussion}

\subsection{Tail Lesions}

The level of pigs with tail lesions in general, was lower in docked than in undocked pigs. Present results underline the findings of Gentz et al. (2019) which referred to a subset of the present data. The paper focussed on a method to analyse an animal individual lesion parameter. This tail biting reducing function of docking is already known in the literature (Lahrmann et al., 2017; Moinard et al., 2003; Schrøder-Petersen and Simonsen, 2001) but is ethically questionable.

In accordance with other studies (Naya et al., 2018; Veit et al., 2016) which detected an increasing number of tail lesions one week after weaning, tail lesions increased in this study in the second week after weaning for all housing and docking groups, but to different extents. Naya et al. (2018) found that pigs seemed to become more courageous from the second week of age and often started to nibble and bite on boots and clothes of the observer. Tail biting may be a compensatory behaviour and occurs if an animal's individual level of compensable stress is exceeded (Schrøder-Petersen and Simonsen, 2001) The coping hypothesis proposes that stereotypies or abnormal behaviour (such as tail biting) reduce the physiological stress reaction linked to environmental impacts (Benus et al., 1991; Rushen, 1993). The peak of the undocked CONV pigs was found after regrouping to fattening, while the peak of the undocked W-F pigs was four weeks later. Thus, the CONV pigs started earlier and showed tail lesions which could be the result of coping of stress (Benus et al., 1991; Rushen, 1993). The undocked CONV pigs showed a sudden increase in week seven, compared to week six which was likely related to the regrouping stress and a higher aggression level (Ekkel et al., 1995). The shifted start of the W-F pigs might be explained by an accumulation of stress over time, whereupon the pigs reacted with increased tail biting in week 11 (Weary et al., 2008). These pigs did not have regrouping stress and stayed in their pens, but the W-F pigs had twice the space allowance of the CONV pigs during rearing. This advantage was obsolete during fattening, which could be stressful and might influence the shift in tail biting towards week 11 as well. Another possible explanation for the increased tail lesion level in the middle of the fattening period might be the onset of sexual maturity (Berry and Signoret, 1984). The animals became more restless due to hormones and their behaviour changes. The housing of intact males caused more mounting behaviour, this resulted in repeated irritation of wounds. The docked animals did not show clear peaks, neither in the W-F nor in the CONV, this could be explained by the fact that they may have different coping strategies than tail biting and this would be beneficial to add in the video analysis. 
Taylor et al. (2012) already showed the impact of a feed change on tail biting (Taylor et al., 2012). In the current study, feed was changed and blended during weeks 1, 7 and 14 to adjust the feed to the needs of the pigs. All changes seemed to affect the pigs in the current study. The tail lesions of the undocked and docked pigs of both rearing groups increased in all three weeks, whereas the changes during week 14 seemed to specifically affect the W-F pigs.

Regarding the level of pigs with tail lesions during the 18 assessment weeks, the undocked W-F pigs were $10 \%$ lower than the undocked CONV pigs. The maximum tail lesion level of the undocked CONV pigs was $20 \%$ higher compared to the undocked W-F pigs. During the rearing period, the pen design of both systems was mainly distinguished by the floor and the space allowance (CONV: 0.44m²; W-F: $0.89 \mathrm{~m}^{2}$ ). While the CONV had plastic, the W-F animals had a concrete floor. Abriel and Jais (2013) have also observed the influence of the external environment on the husbandry system in cannibalism in pigs (Abriel and Jais, 2013a). Already $50 \%$ more space allowance than the legal minimum requirement reduced the level of tail lesions (Abriel and Jais, 2013a).

This method of detecting tail lesions completely disregards the age of the lesion. Consequently, not all recorded lesions occurred at the current week and might be older, but refreshed and assessed previously. This study focused on analysing the percentage of animals with tail lesions and not the percentage of animals with new lesions. To our knowledge, there is no method of tail lesion scoring which includes the evaluation of the age. To counteract this problem, it would be possible to change the frequency of the assessment and score the tails more often.

The batch effect contains all effects which were not analysed separately. In the current study, the differences were maybe caused by learning effects of the staff. Over time, the identification of offenders has been optimised and the timing of the renewal or addition of new enrichment material has also been improved. The prevalence of tail lesions has been reduced by more intensive animal control. The batch effect can also be explained by seasonal changes in the environment. Especially the light conditions (Parker et al., 2010) differ slightly over the year.

The positive effect of early socialization (Cox and Cooper, 2001) has not been shown to have a decisive impact. It seems to have a more important effect on skin lesions and agonistic behaviour of pigs (D'Eath, 2005). Behaviours which are associated with hierarchy and rank fights are affected by socialization (Lange et al., 2018), but tail biting is more clearly influenced by stress and space allowance in the current study. 


\subsection{Tail Losses}

Docked pigs showed no tail losses in this study and needed to be excluded from the statistical analysis. Significant differences for undocked pigs concerning tail losses could be found in the rearing systems. These differences were evident both at the end of the rearing period and at the end of the fattening period. The fact that the W-F pigs suffered fewer losses could result from fewer lesions (average level of pigs with tail lesions CONV $=31.4 \%$; $W-F=23.5 \%$ ). In addition, tail biting was also less intense (low occurrence of severe and very severe lesions (< $2 \%$ ) or differently motivated (Taylor et al., 2010). As Taylor et al. (2010) described, there are several types of tail biting that have different causes. Additional behavioural analysis would be beneficial to verify this. The lower percentage of tail losses of the W-F pigs might also be explained by twice the space allowance at the beginning of rearing compared to the CONV pigs, which allows for the avoidance of biting pigs.

It is noticeable that the relative percentage of tail losses was lower during rearing (6 weeks) than in the fattening period (12 weeks). Tail lesions assessed during the rearing period were almost only slight lesions of the scoring key. In the fattening period, severe or very severe lesions also occurred. Wounds differ in the healing process and often lead to losses only in the case of severe lesions. The type of tail biting could also be an explanation (Taylor et al., 2010). Possibly, the biting behaviour consisted of rather playful chewing during rearing and more aggressive biting during fattening. Additionally, the agonistic interactions in the middle of the fattening period increase with the onset of sexual maturity (Naya et al., 2018). The animals start to mount each other, and lesions lead to losses directly. It might be advantageous to score the pigs tails more often in further studies. The direct link between tail lesions and losses confirms that the recording of tail losses in the slaughterhouse is representative for the assessment of tail biting.

The tail losses of male and female pigs were not significantly different in this study. Female pigs had more intact tails and fewer losses than the males. Zonderland et al. (2010) and Statham et al. (2011) already detected a significant influence of the sex for tail biting (Statham et al., 2011; Zonderland et al., 2010). Female pigs have suffered fewer lesions and losses in these studies.

\section{Conclusions}

The results show that the rearing system affected the tail lesions and losses in the current study. The higher space allowance of the W-F pigs during the rearing period compared with CONV had a positive effect. The W-F curves of tail lesions increased slower and stayed on a lower level which might be caused by a lower stress level due to reduced regrouping. Nevertheless, it must be mentioned that the largest differences were found between docked 
and undocked pigs. Further optimization of housing systems to avoid tail biting as well as other tail biting abnormalities for rearing and fattening of pigs are necessary.

Author Contributions: Conceptualization, M.Ge. and I.T.; methodology, M.Ge., I.T., A.L. and S.Z.; software, M.Ge. and S.Z.; validation, M.Ge., A.L., S.Z., O.B., M.Ga., C.L. and I.T.; formal analysis, M.Ge.; investigation, M.Ge. and A.L.; resources, I.T., O.B.; M.Ga. and C.L.; data curation, M.Ge. and A.L.; writing - original draft, M.Ge.; writing - review and editing, M.Ge., A.L., S.Z., O.B., M.Ga., C.L. and I.T.; visualization, M.Ge. and S.Z.; supervision, I.T.; project administration, I.T., O.B.; M.Ga. and C.L.; funding acquisition, I.T., O.B.; M.Ga. and C.L.; All authors have read and agreed to the published version of the manuscript.

Funding: This research was funded by the Federal Office for Agriculture and Food of Germany and the Landwirtschaftliche Rentenbank (project no.: 2817205413; 758914), and by the H. Wilhelm Schaumann Foundation.

Acknowledgments: Thanks to the Chamber of Agriculture of Schleswig Holstein for their support during the project. We acknowledge support by the German Research Foundation and the Open Access Publication Funds from Göttingen University.

Conflicts of Interest: The authors declare no conflict of interest. The funders did not play a role in the design of the study, in the collection, analyses, or interpretation of data, in the writing of the manuscript, or in the decision to publish the results.

\section{References}

Abriel, M., Jais, C., 2013a. Einfluss der Haltungsbedingungen auf das Auftreten von Kannibalismus bei Aufzuchtferkeln. Landtechnik (68(6)), 389-394.

Abriel, M., Jais, C., 2013b. Mehr Tierwohl - Maßnahmen im Bereich der Haltung: Versuche zur Reduzierung des Schwanzbeißens bei Ferkeln 11, 41-42. Bayrische Landesanstalt für Landwirtschaft, Freising, 95 pp.

Beattie, V.E., Walker, N., Sneddon, I.A., 1996. An investigation of the effect of environmental enrichment and space allowance on the behaviour and production of growing pigs. Applied Animal Behaviour Science (48), 151-158. https://doi.org/10.1016/01681591(96)01031-3.

Benus, R.F., Bohus, B., Koolhaas, J.M., van Oortmerssen, G.A., 1991. Heritable variation for aggression as a reflection of individual coping strategies. Experientia 47 (10), 1008-1019. https://doi.org/10.1007/BF01923336.

Berry, M., Signoret, J.-P., 1984. Sex play and behavioural sexualization in the pig. Reproduction Nutrition Dévelopment (24(5A)), 507-513. 
Bohnenkamp, A.-L., Traulsen, I., Meyer, C., Müller, K., Krieter, J., 2013. Comparison of growth performance and agonistic interaction in weaned piglets of different weight classes from farrowing systems with group or single housing. Animal 7 (2), 309-315. https://doi.org/10.1017/S1751731112001541.

Connor, J.F., 1998. Wean-to-Finish construction alternatives, management, and performance. University of Minnesota, 1998.

Council of European Union, 2008. Council directive 2008/120/EC laying down minimum standards for the protection of pigs of 18 December 2008.

Coutellier, L., Arnould, C., Boissy, A., Orgeur, P., Prunier, A., Veissier, I., Meunier-Salaün, M.-C., 2007. Pig's responses to repeated social regrouping and relocation during the growing-finishing period. Applied Animal Behaviour Science 105 (1-3), 102-114. https://doi.org/10.1016/j.applanim.2006.05.007.

Cox, L.N., Cooper, J.J., 2001. Observations on the pre- and post-weaning behaviour of piglets reared in commercial indoor and outdoor environments. Animal Science 72 (01), 75-86. https://doi.org/10.1017/S1357729800055570.

D'Eath, R.B., 2005. Socialising piglets before weaning improves social hierarchy formation when pigs are mixed post-weaning. Applied Animal Behaviour Science 93 (3-4), 199 211. https://doi.org/10.1016/j.applanim.2004.11.019.

Davis, M.E., Sears, S.C., Apple, J.K., Maxwell, C.V., Johnson, Z.B., 2006. Effect of weaning age and commingling after the nursery phase of pigs in a wean-to-finish facility on growth, and humoral and behavioral indicators of well-being. Journal of Animal Science 84 (3), 743-756. https://doi.org/10.2527/2006.843743x.

Ekkel, E.D., van Doorn, C.E.A., Hessing, M.J.C., Tielen, M.J.M., 1995. The Specific-StressFree Housing System Has Positive Effects on Productivity, Health, and Welfare of Pigs. Journal of Animal Science (73), 1544-1551.

Gentz, M., Lange, A., Zeidler, S., Traulsen, I., 2019. Classification of Pigs with Tail Lesions from Different Farrowing and Rearing Systems during Rearing and Fattening Period. Animals 9 (11). https://doi.org/10.3390/ani9110949.

Grimberg-Henrici, C.G.E., Büttner, K., Lohmeier, R.Y., Burfeind, O., Krieter, J., 2019. The effect of group-housing with free-farrowing pens on reproductive traits and the behaviour of low-risk and high-risk crushing sows. Applied Animal Behaviour Science 211, 33-40. https://doi.org/10.1016/j.applanim.2018.12.001.

Hurvich, C.M., Tsai, C.-L., 1989. Regression and Time Series Model Selection in Small Samples. Biometrika 76 (2), 297. https://doi.org/10.2307/2336663.

Lahrmann, H.P., Busch, M.E., D'Eath, R.B., Forkman, B., Hansen, C.F., 2017. More tail lesions among undocked than tail docked pigs in a conventional herd. Animal 11 (10), 1825-1831. https://doi.org/10.1017/S1751731117000490. 
Lange, A., Lambertz, C., Ammer, S., Gauly, M., Traulsen, I., 2018. Do farrowing and rearing systems affect the agonistic behaviour of pigs at regrouping?, in: Book of abstracts of the 69th Annual Meeting of the European Federation of Animal Science: Dubrovnik, Croatia, 27th-31st August, 2018. 69th Annual Meeting of EAAP, Dubrovnik, Croatia. 27-31 August 2018. Wageningen Academic Publishers, Wageningen, Netherlands, p. 435.

Lohmeier, R.Y., Grimberg-Henrici, C.G.E., Büttner, K., Burfeind, O., Krieter, J., 2020.

Farrowing pens used with and without short-term fixation impact on reproductive traits of sows. Livestock Science 231, 103889. https://doi.org/10.1016/j.livsci.2019.103889.

Meyer-Hamme, S.E.K., Lambertz, C., Gauly, M., 2016. Does group size have an impact on welfare indicators in fattening pigs? Animal 10 (1), 142-149.

https://doi.org/10.1017/S1751731115001779.

Moinard, C., Mendl, M., Nicol, C.J., Green, L.E., 2003. A case control study of on-farm risk factors for tail biting in pigs. Applied Animal Behaviour Science (81 (4)), 333-355. https://doi.org/10.1016/S0168-1591(02)00276-9.

Naya, A., Traulsen, I., Gertz, M., Hasler, M., Burfeind, O., große Beilage, E., Krieter, J., 2018. Is tail biting in growing pigs reduced by a prolonged suckling period? Applied Animal Behaviour Science. https://doi.org/10.1016/j.applanim.2018.10.020.

Oostindjer, M., van den Brand, H., Kemp, B., Bolhuis, J.E., 2011. Effects of environmental enrichment and loose housing of lactating sows on piglet behaviour before and after weaning. Applied Animal Behaviour Science 134 (1-2), 31-41. https://doi.org/10.1016/j.applanim.2011.06.011.

Parker, M., O'Connor, E., McLeman, M., Demmers, T., Lowe, J., Owen, R., Davey, E., Wathes, C., Abeyesinghe, S., 2010. The impact of chronic environmental stressors on the social behaviour of growing pigs, Sus scrofa. Advances in Animal Biosciences 1 (01), 187. https://doi.org/10.1017/S2040470010003304.

Rushen, J., 1993. The "coping" hypothesis of stereotypic behaviour. Animal Behaviour (45, 3).

SAS R Institute Inc, 2008. User's guide (release 9.4). SASR Institute Inc.

Schrøder-Petersen, D.L., Simonsen, H.B., 2001. Tail biting in pigs. Veterinary journal 162 (3), 196-210. https://doi.org/10.1053/tvjl.2001.0605.

Schwarz, G., 1978. Estimating the Dimension of a Model, $1 \mathrm{p}$.

Statham, P., Green, L., Mendl, M., 2011. A longitudinal study of the effects of providing straw at different stages of life on tail-biting and other behaviour in commercially housed pigs. Applied Animal Behaviour Science 134 (3-4), 100-108. https://doi.org/10.1016/j.applanim.2011.08.009.

Taylor, N.R., Main, D.C.J., Mendl, M., Edwards, S.A., 2010. Tail-biting: A new perspective. Veterinary journal 186 (2), 137-147. https://doi.org/10.1016/j.tvjl.2009.08.028. 
Taylor, N.R., Parker, R.M.A., Mendl, M., Edwards, S.A., Main, D.C.J., 2012. Prevalence of risk factors for tail biting on commercial farms and intervention strategies. Veterinary journal 194 (1), 77-83. https://doi.org/10.1016/j.tvjl.2012.03.004.

Valros, A., 2018. Tail biting, in: Špinka, M. (Ed.), Advances in pig welfare. Woodhead Publishing an imprint of Elsevier, Duxford, United Kingdom, 137-166.

van Putten, G., 1969. An Investigation into Tail-Biting among Fattening Pigs. British Veterinary Journal 125 (10), 511-517. https://doi.org/10.1016/S0007-1935(17)48710-0.

Veit, C., Traulsen, I., Hasler, M., Tölle, K., Burfeind, O., grosse Beilage, E., Krieter, J., 2016. Influence of raw material on the occurrence of tail-biting in undocked pigs. Livestock Science 191, 125-131. https://doi.org/10.1016/j.livsci.2016.07.009.

Weary, D.M., Jasper, J., Hötzel, M., 2008. Understanding weaning distress. Applied Animal Behaviour Science 110 (1-2), 24-41. https://doi.org/10.1016/j.applanim.2007.03.025.

Wolter, B.F., Ellis, M., Curtis, S.E., Augspurger, N.R., Hamilton, D.N., Parr, E.N., Webel, D.M., 2001. Effect of group size on pig performance in a wean-to-finish production system. Journal of Animal Science 79 (5), 1067-1073. https://doi.org/10.2527/2001.7951067x.

Wolter, B.F., Ellis, M., DeDecker, J.M., Curtis, S.E., Hollis, G.R., Shanks, R.D., Parr, E.N., Webel, D.M., 2002. Effects of double stocking and weighing frequency on pig performance in wean-to-finish production systems. Journal of Animal Science 80 (6), 1442-1450. https://doi.org/10.2527/2002.8061442x.

Zonderland, J.J., Bracke, M.B.M., den Hartog, L.A., Kemp, B., Spoolder, H.A.M., 2010. Gender effects on tail damage development in single- or mixed-sex groups of weaned piglets. Livestock Science 129 (1-3), 151-158. https://doi.org/10.1016/j.livsci.2010.01.018.

Zonderland, J.J., Wolthuis-Fillerup, M., van Reenen, C.G., Bracke, M.B.M., Kemp, B., Hartog, L.A.d., Spoolder, H.A.M., 2008. Prevention and treatment of tail biting in weaned piglets. Applied Animal Behaviour Science 110 (3-4), 269-281. https://doi.org/10.1016/j.applanim.2007.04.005. 


\section{PAPER TWO}

\section{Classification of pigs with tail lesions from different farrowing and rearing systems during rearing and fattening period}

Maria Gentz ${ }^{1, \star}$, Anita Lange ${ }^{1}$, Sebastian Zeidler ${ }^{2}$ and Imke Traulsen ${ }^{1}$

1 Department of Animal Sciences, Livestock Systems, Georg-August-University, Albrecht-Thaer-Weg 3, 37075 Göttingen, Germany; anita.lange@agr.unigoettingen.de (A.L.); imke.traulsen@uni-goettingen.de (I.T.)

2 Department of Animal Sciences, Breeding Informatics, Georg-August-University, Margarethe-von-Wrangell-Weg 7, 37075 Göttingen, Germany; sebastian.zeidler@uni-goettingen.de (S.Z.)

*Correspondence: maria.gentz@uni-goettingen.de (M.G.) 


\section{Simple Summary}

Tail biting is a well-known problem in pig production, whereby animals with tail lesions suffer from pain, infections, and reduced feed intake. Controlling tail biting could have a positive effect on animal welfare and on the economic viability of farms. A classification of typical traits of pigs with tail lesions could provide the opportunity to protect the pigs. A combined parameter based on frequency and duration of tail lesions was created to find out whether biologically relevant groups can be separated by cluster analysis. The results show that the created lesion parameter was suitable to describe the degree of impairment of the pigs. However, not all pigs were affected to the same extent by tail biting. The high impact of the docking status and the reduction of tail lesions by more space allowance was shown.

\section{Abstract}

The aim of the present study was to classify and characterise pigs with tail lesions using a combined parameter based on the frequency and duration of tail lesions and to find out whether biologically relevant groups could be separated by cluster analysis. Pigs ( $n=677,50 \%$ docked, $50 \%$ undocked) from three farrowing systems, as follows: (1) Conventional farrowing crate (FC), (2) free farrowing (FF), and (3) a group housing lactating sows $(\mathrm{GH})$, were divided into two rearing systems as follows: (1) A conventional system (CONV) and (2) a wean-to-finish $(\mathrm{W}-\mathrm{F})$ system. Within 18 assessment weeks, starting after weaning, animal tail lesions were recorded individually. The animals were characterised into five lesion groups, as follows: (I) No lesions to $(\mathrm{V})$ many long lasting lesions. The separability of the predefined lesion groups was checked by an animal individual lesion parameter. By using a k-means cluster analysis, it was shown that the docking status was the mainly affected parameter on the tail lesions. The separation of the groups only succeeded for the most distinct groups, I and V. The high impact of the docking status and the reduction of tail lesions by more space allowance was shown. More characterising information for the individual pigs would improve the separability of the lesion groups.

Keywords: classification; tail lesions; pigs; rearing system; farrowing system; docked tails; undocked tails; stress; tail biting; cluster analysis 


\section{Introduction}

Tail biting is a huge and well-known problem in pig production. A lesion can range from mild bite marks, with or without punctuation of the skin, up to a complete tail loss (Valros, 2018). If a tail lesion occurs, it results in pigs suffering from pain, and more symptoms, e.g., reduced weight gain and a higher risk of infection diseases, which results in a need for medical treatment (Schrøder-Petersen and Simonsen, 2001; Taylor et al., 2012). Tail lesions can also lead to economic losses at slaughter, carcass condemnation, and secondary infections (Hunter et al., 1999). Pigs show individual reactions to different stressors such as climatic and light conditions (Parker et al., 2010), sex (Zonderland et al., 2010), husbandry environment (Cox and Cooper, 2001), feeding (Statham et al., 2011), and group size (Meyer-Hamme et al., 2016), among others. Tail biting does not necessarily lead to lesions. Taylor et al. (2010) (Taylor et al., 2010) described three different types of tail biting behaviour, whereby the first type describes a gentle and harmless chewing on the tail of another pig, where the tail remains complete and shows no lesions (Schrøder-Petersen and Simonsen, 2001), but can also result in tail lesions which can be responsible for a tail biting outbreak (Statham et al., 2009; Taylor et al., 2010). The second type of tail biting is energetic tail biting, which can be triggered by deficiencies in the abundance or accessibility of water, feeding, or space. The third type is a compulsive and abnormal behaviour of individual animals whose cause is still unknown (Taylor et al., 2010).

In order to work out profiles within groups, a cluster analysis is often carried out (Lekic and Romanowicz, 2011; Lukashin and Fuchs, 2001). For example, in human fields, this method is used in gene expression analysis (Lukashin and Fuchs, 2001), thermography (Lekic and Romanowicz, 2011), or for motivation profiles of athletes (Zason Chian and John Wang, 2008). The k-means cluster algorithm is used to find optimal distribution profiles or to work out specific character traits (Lekic and Romanowicz, 2011; Lukashin and Fuchs, 2001). In addition, the relationship between single groups can be ranged (Zason Chian and John Wang, 2008). A kmeans cluster analysis is preferred, as this method is particularly suitable for large amounts of data and the number of clusters generated can be predefined. After cluster analysis, the identified relevant groups can be analysed in detail by several tests to separate and describe these groups.

Different types of tail biting can lead to different types of wounds (Taylor et al., 2010). There are small wounds that heal up quickly and large wounds that require more time (Taylor et al., 2010; van Putten, 1969). Consequently, the degree and duration of tail lesions differ more or less. This means that pigs can be divided into animals without lesions, with few, and with many tail lesions. In addition, the intensity of the wound determines the duration of healing. 
A reduction of stressful factors and an optimisation of the housing conditions can increase animal welfare and help to reduce tail biting as a coping strategy (Benus et al., 1991). For example, early socialisation was shown to help piglets to handle stressful situations better (D'Eath, 2005). Farrowing and rearing systems with a higher space allowance, and without any fixation of the sow, may improve the socialisation level of piglets and the welfare of sows (Oostindjer et al., 2011).

The aim of this study was to classify and characterise pigs using a combined parameter, based on their frequency and duration of tail lesions, and to find out whether biologically relevant groups could be separated by cluster analysis. As influencing factors, docking status, rearing system, farrowing system, and weights at weaning as well as at the end of rearing were considered.

\section{Materials and Methods}

\subsection{Animals and Housing}

The dataset comprised measurements of 1252 pigs, where 677 animals provided complete measurements in 18 subsequent weeks. The data was acquired at the research farm Futterkamp at the chamber of Agriculture of Schleswig-Holstein, located in northern Germany. Within seven batches, 309 pigs were housed with docked tails (45.64\%) and 368 pigs were left undocked (54.36\%). Males were raised as intact males. For farrowing, sows were allocated to three farrowing systems, as follows: A conventional farrowing crate (FC), a free farrowing system (FF), and a group housing system of lactating sows (GH) (Figure 1). In the FC and FF systems, the sows were single housed. In the FC, sows were fixed permanently, and in the FF system, sows could move freely and interact with their piglets, except for one batch, in which the sows were fixed for three days post-partum to minimise the crushing of the piglets (piglets treated as being FF). In the GH system, ten sows were housed together. Until five days postpartum, the sows were single housed in a free-farrowing pen. Afterwards, the sows and their piglets were commingled, joining a running area and their pens. 


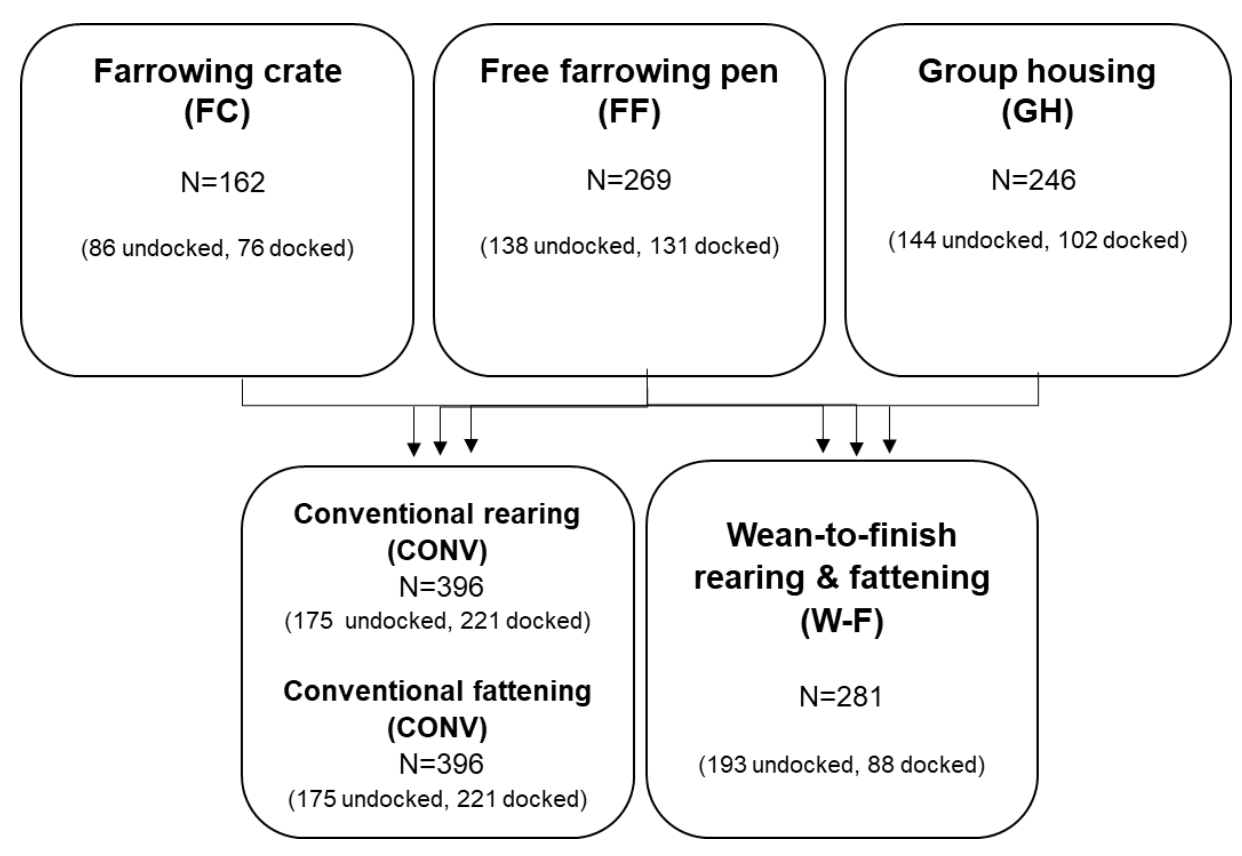

Figure 1. Schematic overview of the $3 \times 2 \times 2$ factorial experimental design.

Figure 1 shows an overview of the $3 \times 2 \times 2$ factorial experimental design. After 27 days postpartum, the piglets were weaned and divided randomly within the farrowing system into two different rearing systems, as follows: A conventional rearing system ( $\left.\mathrm{CONV}_{\text {rearing }}, 0.44 \mathrm{~m}^{2} / \mathrm{pig}\right)$ and a wean-to-finish system (W-F, $\left.0.89 \mathrm{~m}^{2} / \mathrm{pig}\right)$. The W-F pigs were grouped by sex and the $\mathrm{CONV}_{\text {rearing }}$ pigs were grouped mixed-sex. Pigs of the CONV rearing group were regrouped and rehoused for finishing and sorted by sex after 40 days of rearing $\left(C O N V_{\text {fattening }}\right)$, while the W-F pigs were raised as intact groups until the end of the study (week 18 after weaning). The $\mathrm{CONV}_{\text {fattening }}$ pigs had $0.89 \mathrm{~m}^{2}$ per pig and their pens were identical in structure to those of the $\mathrm{W}-\mathrm{F}$ pens. In $\mathrm{CONV}_{\text {rearing, }}$, the piglets were housed in groups of 13 pigs and in $\mathrm{W}-\mathrm{F}$, the piglets were housed in groups of 14 pigs. The slat width on all floors $(13 \mathrm{~mm}$, tread area $=67 \mathrm{~mm})$ was determined in a special permit (V242-226720/2015 (8-1/16)) to allow the pigs to be kept in the same pens from weaning to the end of fattening. The pigs were fed ad libitum with conventional dry feed adjusted to their age. During their whole life, the pigs always had access to at least one or several different enrichment materials; for example, pieces of wood, ropes, jute bags, or troughs filled with dried peas and grass pellets. When an outbreak of tail biting occurred, pigs were offered additional or renewed enrichment material. Additionally, victims of tail biting that were severely injured were excluded from the study $(N=19)$. Evident offenders were also rehoused and excluded $(\mathrm{N}=9)$. Pigs with medical treatable lesions stayed in their pens and were scored consecutively. More details on housing systems can be found in Gentz et al. (2019).

All of the animals were housed in accordance with EU (European Directive 2008/120/EC) and national law (Animal Welfare Act (18/05/2016), Animal Welfare and Animal Husbandry Ordinance (05/02/2014)). 


\subsection{Data Collection}

The weaning weight and the 40-days-weight were collected individually. The two weights were included in the evaluation.

Starting one day after weaning, the piglets' tails were scored individually once a week until assessment at week 18 using a modified Schwarzenauer key (Abriel and Jais, 2013). From the collected data, the tail lesions were used for the present study. A tail "lesion" means that the tail has slight scratches or bite marks, a "severe lesion" shows deeper flat lesions, and a "very severe lesion" would be a deep flat lesion which is greater than $2 \mathrm{~cm}$.

For the statistical analysis, the tail lesion score was summarised due to the low occurrence of severe lesions into "no lesions" (0) and "lesions" (1). Each pig was scored for each assessment week, whether a tail lesion was observed or not.

\subsection{Statistical Analysis}

The statistical language R (R Core Team, 2016) was used to analyse the collected data. The statistical analysis was conducted in different steps, which are explained in the following scheme (Figure 2) (oriented at the method of Lukashin and Fuchs (2001)).

\section{Preprocessing}

Step 1: Creating lesion groups

Method: Animal individual seperation, descriptive analysis of the sum and the maximum duration of tail lesions

\section{Verification and Cluster analysis}

Step 2: Verification of the separability of the lesion groups Method: Creating a lesion parameter, boxplot and cluster analysis

Step 3: Cluster analysis of the groups

Method: K-means cluster analysis (2 clusters)

Step 4: Comparison of the groups

Method: T-test

Figure 2. Workflow of the statistical analysis. 


\subsubsection{Preprocessing}

\section{Step 1: Creating Lesion Groups}

The aim of this step was to create a single animal individual lesion parameter. First, an analysis of the frequency of score "1" and the maximum duration of consecutive score "1" was carried out (Table 1). The frequency of score "1", which represents the sum of score "1" over all 18 assessment weeks, was calculated. In addition, the maximal duration of consecutive score "1" was calculated. This was used as an indicator of the impact of tail lesions. Table 1 shows an example for the scoring of five different pigs.

Table 1. Examples of the tail lesion scoring, frequency of score "1", and maximum duration ( 1 = a tail lesion was scored; $0=$ no tail lesion was scored $).{ }^{1}$ Frequency of score " 1 "; ${ }^{2}$ maximum duration of score " 1 ".

\begin{tabular}{|c|c|c|c|c|c|c|c|c|c|c|c|c|c|c|c|c|c|c|c|c|}
\hline & \multicolumn{18}{|c|}{ Assessment weeks } & \multirow[t]{2}{*}{ Freq. ${ }^{1}$} & \multirow{2}{*}{$\begin{array}{l}\text { Max. } \\
\text { dur. }\end{array}$} \\
\hline & 1 & 2 & 3 & 4 & 5 & 6 & 7 & 8 & 9 & 10 & 11 & 12 & 13 & 14 & 15 & 16 & 17 & 18 & & \\
\hline Pig 1 & 0 & 0 & 0 & 0 & 0 & 0 & 0 & 0 & 0 & 0 & 0 & 0 & 0 & 0 & 0 & 0 & 0 & 0 & 0 & 0 \\
\hline Pig 2 & 0 & 0 & 0 & 1 & 0 & 0 & 0 & 1 & 0 & 0 & 0 & 1 & 0 & 0 & 0 & 0 & 0 & 0 & 3 & 1 \\
\hline Pig 3 & 0 & 0 & 0 & 0 & 0 & 1 & 1 & 0 & 0 & 0 & 0 & 1 & 0 & 0 & 0 & 0 & 0 & 0 & 3 & 2 \\
\hline Pig 4 & 0 & 0 & 0 & 0 & 0 & 0 & 0 & 1 & 1 & 1 & 0 & 0 & 0 & 0 & 1 & 0 & 0 & 0 & 4 & 3 \\
\hline Pig 5 & 1 & 1 & 1 & 1 & 1 & 1 & 1 & 1 & 1 & 1 & 0 & 0 & 1 & 1 & 0 & 1 & 0 & 1 & 14 & 10 \\
\hline
\end{tabular}

Pig 1 had no score "1" over all the assessment weeks, which means that the frequency of score "1" was zero and the maximum duration as well. For pig 2, three single scores of "1" were observed, which led to a frequency of score "1" of three and a maximum duration of one, because there was no more score "1" in a row. Pig 3 had a score of "1" during weeks six, seven, and twelve. This means that the frequency of score "1" was three and the maximum duration, as there were two successive score "1" during weeks six and seven, would be two. Pig 4 had three successive and one single score "1" (frequency = 4; maximum duration = 3 ). Pig 5 had a frequency of score " 1 " of 14 and a maximum duration of ten. This means that not all 14 scores of " 1 " were in successive weeks, but ten were successive and four were at any other time.

Figure 3 shows the distribution of the frequency of score "1". A total of $77.4 \%$ of the pigs had five or less scores of " 1 " and $22.6 \%$ suffered from more scores of " 1 ". Less than ten percent of 
the pigs did not have any score " 1 " at all. The percentage of pigs with ten or more scores of "1" was $3.9 \%$.

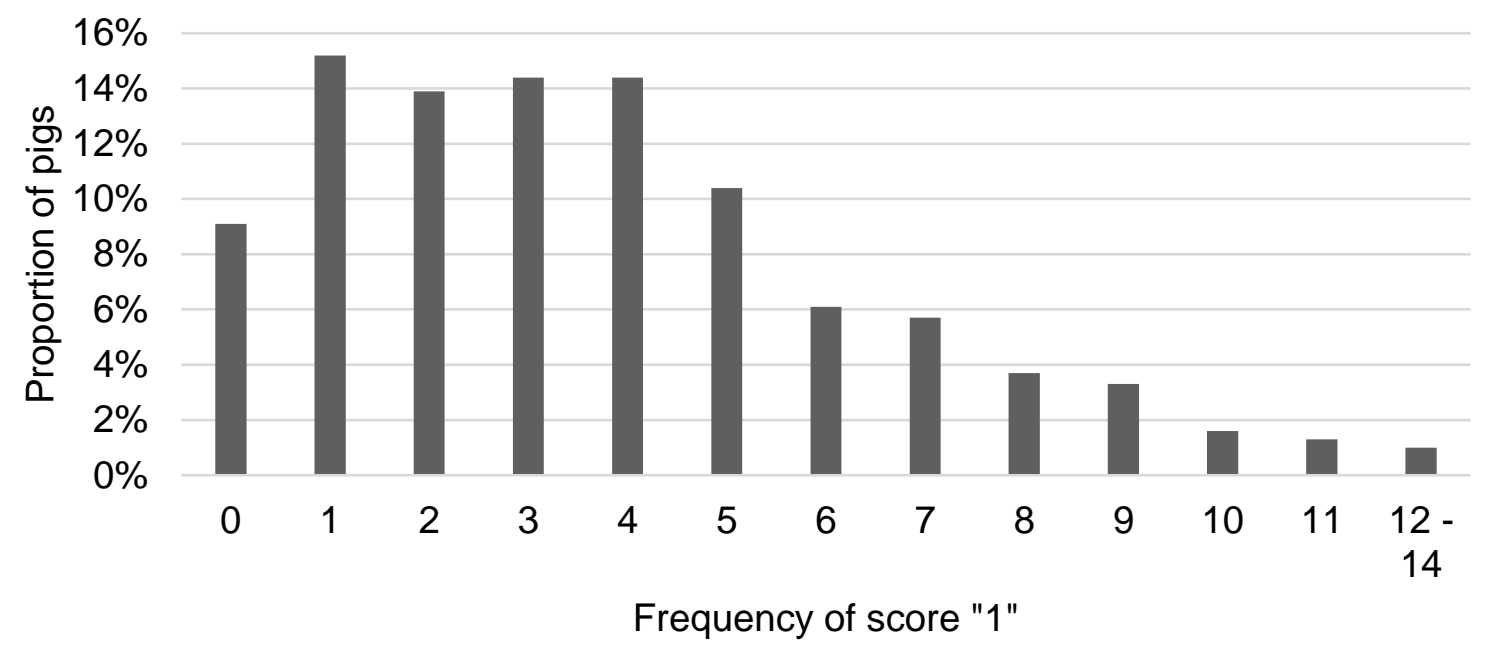

Figure 3. Distribution of the frequency of score "1" of tail lesions per pig over rearing and finishing periods.

In order to separate these pigs, the maximum duration score was used to determine how many consecutive weeks a pig had score "1".

Figure 4 shows the distribution of the maximum duration of a tail lesion score "1". Scores of "1" that lasted longer than six and for a maximum of ten weeks were summarised due to rareness.

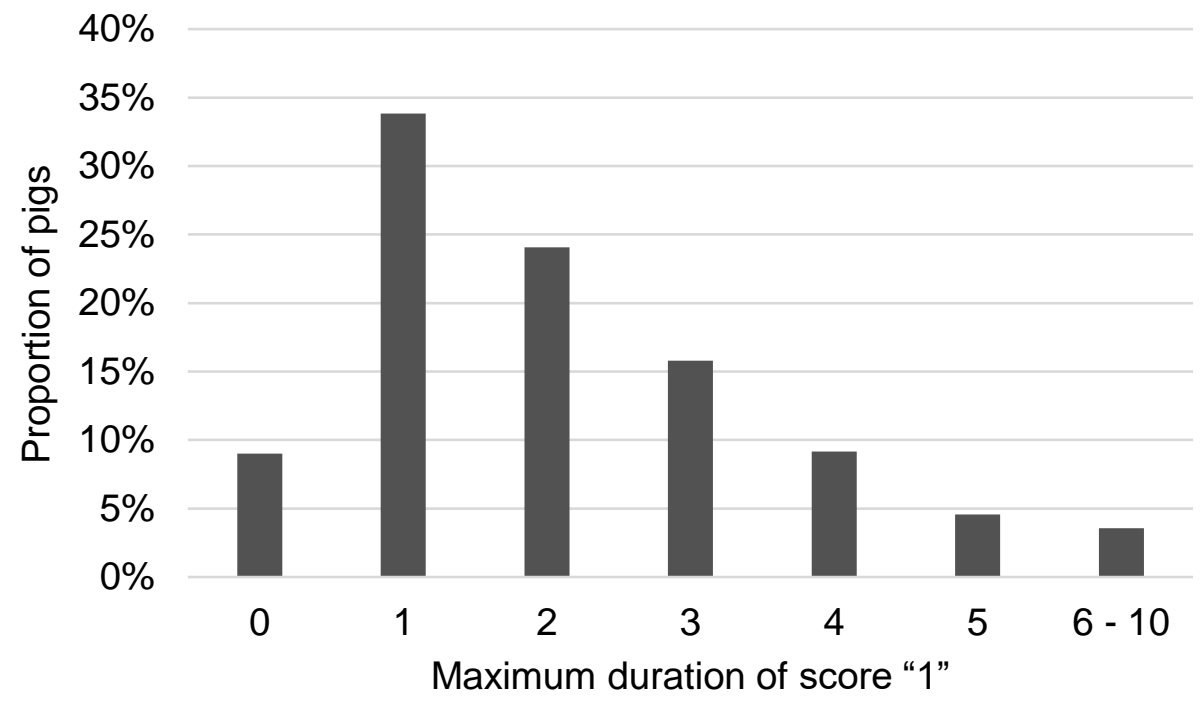

Figure 4. Distribution of the maximum duration of score "1" per pig over rearing and finishing periods.

Based on the frequency of score "1" and the maximum duration, five lesion groups were formed (Table 2). Each pig could only be found in one group. The primary criterion for the group was the frequency of score "1" and the maximum duration worked as second criterion. The duration 
was divided into short and long, whereby short meant $1 / 3$ of the maximum duration within their lesion group and long meant 2/3 corresponding to the maximum duration within their lesion group. Therefore, the pigs with tail lesions could be divided into the following groups: (I) No lesions, (II) few short lesions, (III) few long-lasting lesions, (IV) many short lesions, and (V) many long-lasting lesions.

Table 2. Lesion groups (LG) built of the frequency of score "1" and the maximum duration of score "1". Note that each pig was in one group only.

\begin{tabular}{|c|c|c|c|}
\hline Groups & $\begin{array}{l}\text { Freqency of } \\
\text { score "1" }\end{array}$ & $\begin{array}{l}\text { Max. duration } \\
\text { of score "1" }\end{array}$ & $\mathrm{N}^{1}$ \\
\hline I No lesions & 0 & 0 weeks & 61 pigs \\
\hline $\begin{array}{l}\text { II Few lesions of short duration } \\
\text { III Few lesions of long duration }\end{array}$ & $1-3$ & $\begin{array}{c}1 \text { week } \\
2-3 \text { weeks }\end{array}$ & $\begin{array}{l}204 \text { pigs } \\
90 \text { pigs }\end{array}$ \\
\hline $\begin{array}{l}\text { IV Many lesions of short duration } \\
\text { V Many lesions of long duration }\end{array}$ & $4-14$ & $\begin{array}{l}1-3 \text { weeks } \\
4-10 \text { weeks }\end{array}$ & $\begin{array}{l}205 \text { pigs } \\
117 \text { pigs }\end{array}$ \\
\hline
\end{tabular}

118 assessment weeks for each pig; Exemplary pigs: See Table 1 (LG I Pig 1, LG II Pig 2, LG III Pig 3, LG IV Pig 4, and LG V Pig 5).

The first group consisted of pigs without tail lesions, meaning no scores of " 1 " over all the 18 assessment weeks (I: 61 pigs, 9.01\%). In the second and third group, there were pigs with few tail lesions (frequency of score "1": 1-3) (II: 204 pigs, 30.13\%; III: 90 pigs, 13.29\%) and the fourth and fifth group consists of pigs with many tail lesions (frequency of score "1": 4-14) (IV: 205 pigs, $30.28 \%$; V: 117 pigs, $17.28 \%$ ).

\subsubsection{Cluster Analysis}

Step 2: Verification of the Separability of the Lesion Groups

In Step 2, the separability of the predefined lesion groups was checked. To classify biologically meaningful groups associated with the used docking status, the rearing and the farrowing systems, the lesion groups had to be mostly non-overlapping. In order to test the separability of the individual lesion groups, an animal individual lesion parameter $(\lambda)$ was calculated.

$$
\lambda=\left\{\begin{array}{c}
0, \text { if frequency of score " } 1 "=0 \wedge \text { maximum duration }=0 \\
\frac{\text { frequency of score " } 1 "+\text { maximum duration }}{\text { frequency of score "1" }}
\end{array}\right.
$$

Figure 5 shows a boxplot of the lesion parameter for the different lesion groups. 


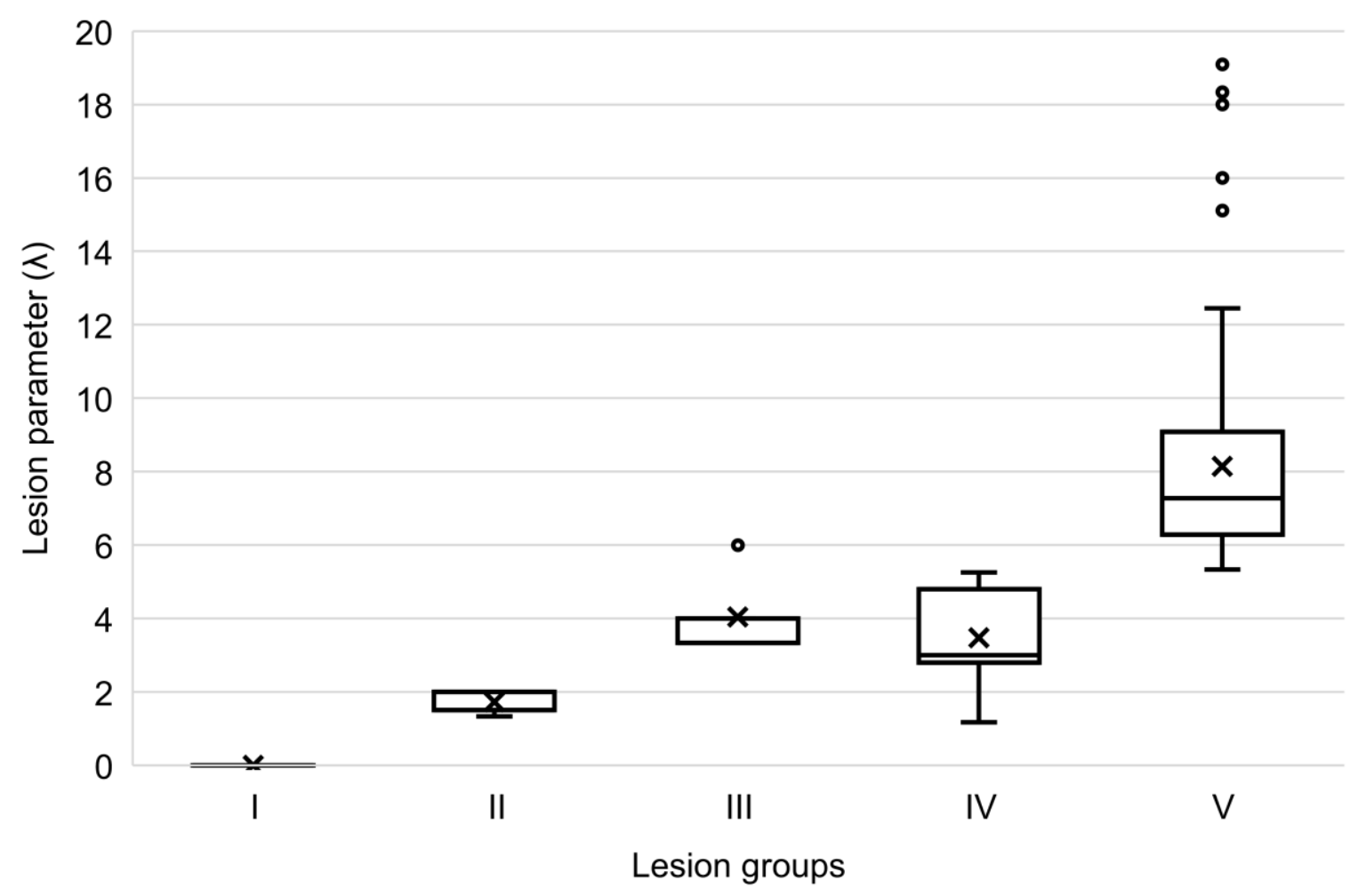

Figure 5. Boxplot analysis of the lesion parameter $(\lambda)$ for each lesion group.

The boxplots show that groups I and V were completely separable from each other. Groups II, III, and IV were difficult to distinguish due to their overlapping lesions scores. In addition, the k-means-clustering algorithm ( $k=5,1000$ iterations) was applied on the data to check for the plausibility of the separation and to validate the lesion groups. The silhouette plot (Figure 6a) underlines the difficulties in separating the five groups, whereas cluster groups 2-4 show low average silhouette scores and cluster group 5 shows negative silhouette scores. To improve the degree of separation, the clusters were modified (increased and decreased), which showed an improved separability in the case of two clusters only. The subsequent analysis was focused on groups I and $\mathrm{V}$, which represented no lesion animals and animals with many long-lasting scores of "1" (Figure 6b). 


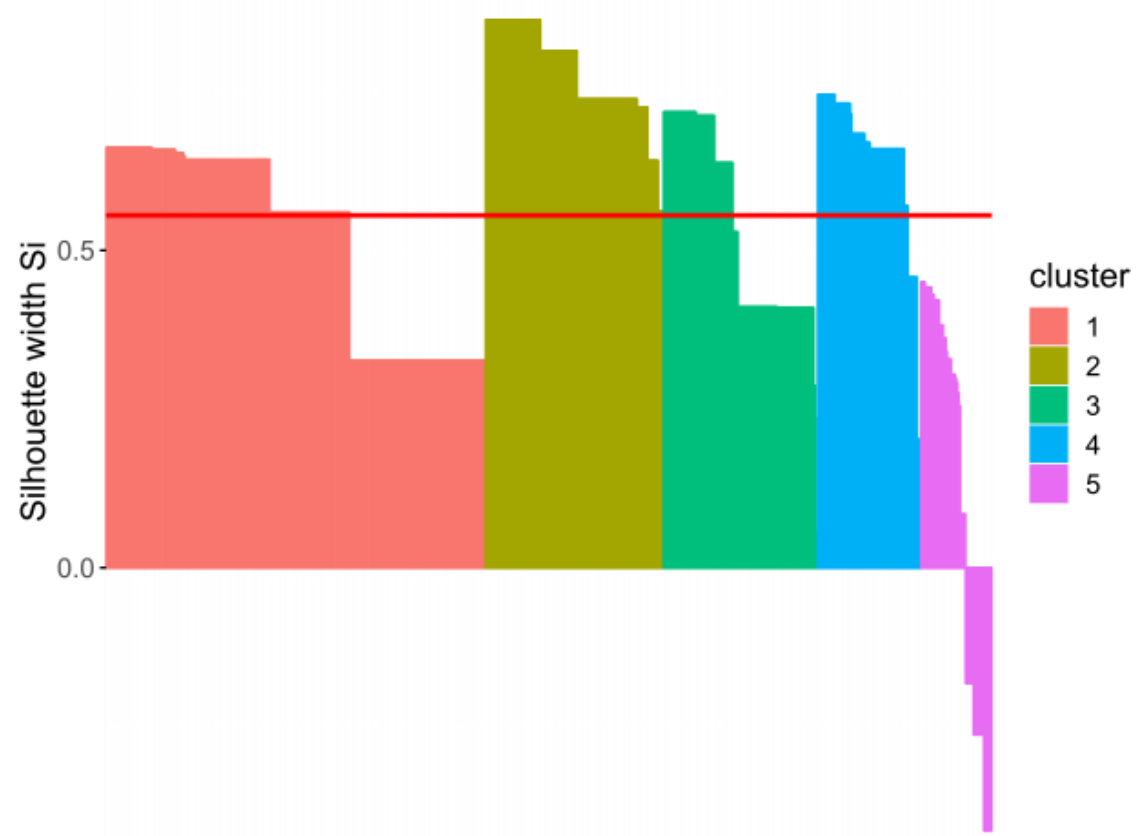

(a)

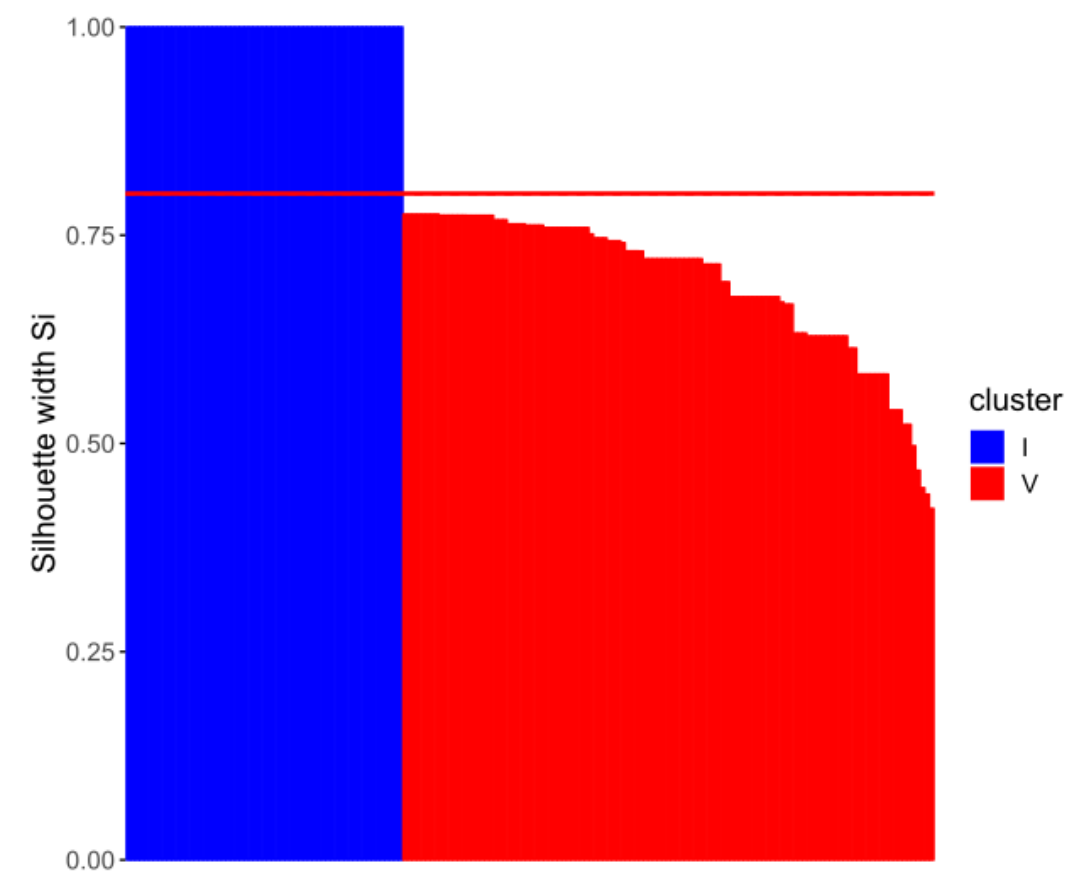

(b)

Figure 6. (a) Silhouette plot for five k-means cluster groups to show the non-separability. The red line shows the average quality of the clusters. (b) Silhouette plot for two k-means cluster groups to show the separability. The red line shows the average quality of the clusters.

Step 3: Cluster Analysis of Most Distinct Groups

With help of $\lambda$, a k-means cluster analysis (Hartigan and Wong, 1979; R Core Team, 2016) for groups I and V was conducted. This iterative method of minimizing the within-class sum of 
squares for a given number of clusters was used to test the classification into the specific lesion groups. The visualization was conducted using the cluster package (Maechler et al., 2012). Figure $6 \mathrm{~b}$ shows, that the data can be separated in two distinct groups, which represent the two lesion groups I and V (see Appendix Figure A1).

Step 4: Comparison of the Non-Overlapping Groups

In the last step, the non-overlapping groups I and V were compared. The docking status, the rearing system, and the farrowing systems were analysed according to the relative frequency of pigs. In order to investigate the significant differences of the weights at weaning and after rearing a t-test was applied.

\section{Results}

The distribution of pigs within the lesion groups (I and V) per docking status, rearing system, and farrowing system is shown in Table 3. Group I consisted of twice as many docked than undocked pigs, whereas group $V$ shows a reversed distribution. The $X^{2}$ test showed that groups $\mathrm{I}$ and $\mathrm{V}$, as well as the docked and undocked pigs, were significantly different $(p<0.05)$. In general, the distribution of the pigs within group $\mathrm{V}$ of the rearing and farrowing systems of group V were very similar (Rearing system: CONV $=16.67, \mathrm{~W}-\mathrm{F}=18.15 \%$; farrowing system: $\mathrm{FC}=15.43, \mathrm{GH}=17.07, \mathrm{FF}=18.59 \%$ ). There were more $\mathrm{W}-\mathrm{F}$ pigs than CONV pigs in each group. The proportion of $\mathrm{FC}$ and $\mathrm{GH}$ pigs in group I was lower than that in group V. Among the FF pigs, group $\mathrm{V}$ contained about one-third more animals than group I.

Table 3. Distribution of the characteristics of the pigs within the lesion groups I and $\mathrm{V}$, related to all pigs of the specific group (Figure 1). Note that the significant differences were calculated with absolute numbers of animals.

\begin{tabular}{ccccc}
\hline & & \multicolumn{3}{c}{ Lesion Group } \\
\cline { 3 - 5 } & & $\mathbf{I}$ & $\mathbf{I I}-\mathbf{~ I V}$ & V \\
& & $(\mathbf{n}=\mathbf{6 1})$ & $\mathbf{( n = 4 9 9 )}$ & $(\mathbf{n}=\mathbf{1 1 7})$ \\
\hline \multirow{2}{*}{ Docking status } & undocked & $5.43 \%^{\mathrm{a}}$ & $83.16 \%$ & $11.41 \%^{\mathrm{b}}$ \\
& docked & $13.27 \%^{\mathrm{c}}$ & $80.26 \%$ & $6.47 \%^{\mathrm{d}}$ \\
\hline \multirow{2}{*}{ Rearing system } & CONV & $6.57 \%$ & $76.76 \%$ & $16.67 \%$ \\
& W-F & $12.46 \%$ & $69.39 \%$ & $18.15 \%$ \\
\hline \multirow{2}{*}{ Farrowing system } & FC & $6.79 \%$ & $77.78 \%$ & $15.43 \%$ \\
& FF & $11.90 \%$ & $69.51 \%$ & $18.59 \%$ \\
& GH & $7.32 \%$ & $75.61 \%$ & $17.07 \%$ \\
\hline
\end{tabular}

${ }^{a-d}$ Different letters indicate significant differences of the groups $(p<0.05)$. 
The comparison of the different weight groups only resulted in significant differences in the group of undocked-CONV-FF animals with regard to the average weaning weights $(I=6.63$ $\mathrm{kg}, \mathrm{V}=8.35 \mathrm{~kg})$ and the average weight at the end of rearing $(\mathrm{I}=22.00 \mathrm{~kg}, \mathrm{~V}=26.40 \mathrm{~kg})$, where the pigs of lesion group $\mathrm{V}$ were heavier. No other animals comparing group I and $\mathrm{V}$ differed significantly. The average weaning weight was $7.96 \mathrm{~kg}(\min =6.35 \mathrm{~kg}, \max =9.35 \mathrm{~kg})$ and the average difference between groups I and $V$ was $0.93 \mathrm{~kg}$. Regarding the weight at the end of rearing, the average weight was $25.18 \mathrm{~kg}(\min =21.20 \mathrm{~kg}$, $\max =28.50 \mathrm{~kg})$, whereby the groups I and $V$ differed by $1.33 \mathrm{~kg}$ on average.

\section{Discussion}

The lesion parameter $\lambda$ was used as a continuous and non-prioritising parameter to define the intensity of tail lesions. It combined the frequency and the duration of tail lesions on an equal level. This parameter might be a useful help to analyse tail lesions in further studies.

A cluster analysis is often used for profiling (Lekic and Romanowicz, 2011; Lukashin and Fuchs, 2001; Zason Chian and John Wang, 2008), as was also done in the present study. The results show that a reliable clustering could only be performed in groups I and V, where the differences of the frequency and the durations were the highest. Groups II, III, and IV were too similar to work out specific traits. In order to figure out the impact of the individual animals and their tail lesions, other traits may have to be considered.

The predefinition of the number of clusters gave the possibility to investigate a specific biologically grounded hypothesis (Sparks et al., 2012). In an experimental design without expectation of a number of clusters, this analysis requires a longer preliminary work. This includes first hierarchical clustering followed by non-hierarchical clustering (Zason Chian and John Wang, 2008). In this study, the k-means clustering made it possible to separate very similar groups respectively to emphasise the separable groups.

Although a total of 1252 fattening pigs were investigated in this study, it was only possible to examine 677 pigs after strict filtering. To assure a representative animal number in both groups $(\mathrm{I}, \mathrm{V})$, an enormous number of assessed animals or simulating techniques might be an option. In addition, it should be noted that the numbers of pigs were not balanced concerning the distribution among the husbandry systems, as well as the docking status, and this leads to limitations in the interpretation of the results. The unbalanced data set influenced the comparison of the lesion groups. The very dominant effect of the docking status covered the other effects. An investigation of only undocked pigs might have resulted in other significances, but the low number of animals would have led to other methodical problems. This method could be improved by using more animal individual characterising traits, such as behaviour or genetics, to make them unique. Since various factors affect the pigs and tail biting is a 
multifactorial problem, it is difficult to determine the most influential traits to avoid tail lesions (Schrøder-Petersen and Simonsen, 2001).

Docking is one of the most commonly used methods to reduce tail biting (Schrøder-Petersen and Simonsen, 2001). It reduces the risk for tail biting related lesions but can neither prevent it completely (Moinard et al., 2003) nor eliminate the causes for the abnormal behaviour. The literature still does not provide clear reasons why docked pigs get less tail lesions (Valros, 2018), but one hypothesis is nerve regeneration. Docking creates a higher sensitivity at tail ends and this benefits in a quicker reaction (Herskin et al., 2015; Simonsen et al., 1991). In addition, the attractiveness of a docked tail is lower (Paoli et al., 2016). The significant effect of tail docking might cover the effects of the rearing and the farrowing system. Nevertheless, differences (not significant) within the husbandry systems show, which might be the better way of housing.

Findings for W-F pigs were in line with the literature. Pigs which were not regrouped and not rehoused had fewer tail lesions and might suffer from less stress, resulting in fewer tail lesions (Ekkel et al., 1995). The present study can support the results of Beattie et al. (1996) about increasing space allowance, which resulted in a reduction of tail biting behaviour among growing pigs (Beattie et al., 1996; Schrøder-Petersen and Simonsen, 2001). The advantages of higher space allowance and loose housing can also be seen from the FF pigs. The frequency of FF pigs in group I was particularly high, which led to the conclusion that the farrowing system has a positive effect on how piglets dealt with their post-weaning environment (Oostindjer et al., 2011). Better socialisation of the piglets also seemed to result in a reduction of aggression in their future life (D'Eath, 2005).

With regard to tail biting, future studies should include more information about the age of the wound to distinguish fresh from old healing or reopened lesions to gather information about the healing process. This would offer the possibility for further improvement in the analysis, especially to analyse whether a pig gets new lesions every week or if there are problems with wound healing.

\section{Conclusions}

This study shows that not all pigs were affected to the same extent by tail biting, which can generally be measured by the lesion parameter. Groups of pigs without tail lesions could be separated from pigs with lesions. The cluster analysis can help to analyse and separate the lesion groups. To enhance the analysis, a larger dataset with only undocked pigs is needed. The availability of more individual animals and unique traits might improve the applicability of the present analysis procedure. Although several traits of the pigs with tail lesions were 
assessed, it was only possible to name significant differences regarding docking status to characterise the groups.

Author Contributions: Conceptualisation, M.G. and I.T.; Data curation, M.G. and A.L.; Formal analysis, M.G.; Funding acquisition, I.T.; Investigation, M.G. and A.L.; Methodology, M.G. and S.Z.; Project administration, I.T.; Resources, M.G. and I.T.; Software, M.G. and S.Z.; Supervision, I.T.; Validation, M.G., A.L., S.Z. and I.T.; Visualisation, M.G. and S.Z.; Writing original draft, M.G.; Writing review \& editing, A.L., S.Z. and I.T.

Funding: This research was funded by the Federal Office for Agriculture and Food of Germany and the Landwirtschaftliche Rentenbank (project no.: 2817205413; 758914) and by the H. Wilhelm Schaumann Foundation.

Acknowledgments: Thanks to the Chamber of Agriculture of Schleswig Holstein and Onno Burfeind for their support during the project. We acknowledge support by the German Research Foundation and the Open Access Publication Funds of the Göttingen University.

Conflicts of Interest: The authors declare no conflict of interest. The funders did not play a role in the design of the study, in the collection, analyses, or interpretation of data, in the writing of the manuscript, or in the decision to publish the results. 


\section{Appendix A}

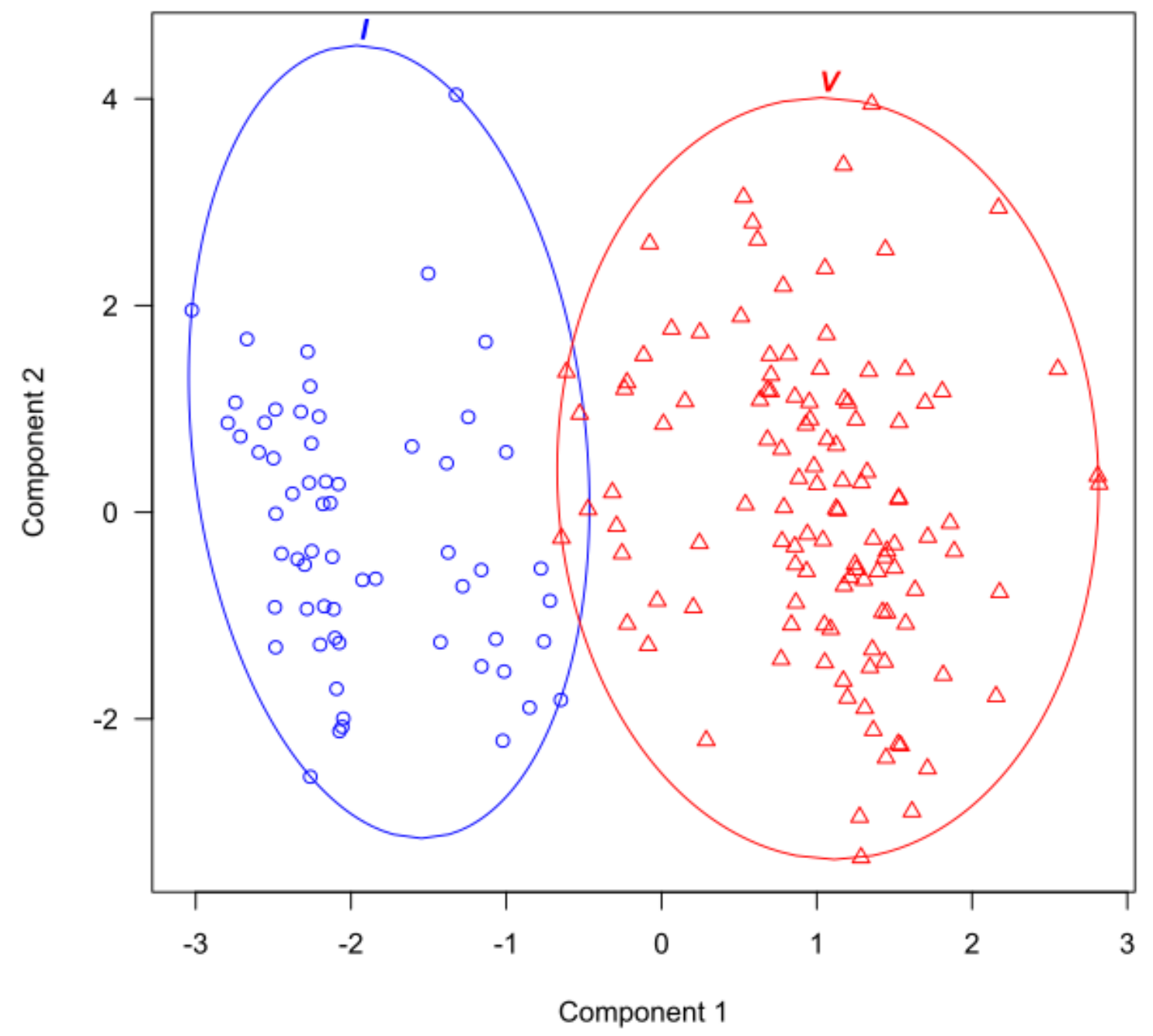

Figure A1. Cluster plot of the two separated groups I (blue) and V (red). The cluster analysis resulted in two nearly non-overlapping groups. The pigs without lesions (group I) were clearly separable from the pigs with many long-lasting scores of "1" (group V). 


\section{References}

Abriel, M., Jais, C., 2013. Mehr Tierwohl - Maßnahmen im Bereich der Haltung: Versuche zur Reduzierung des Schwanzbeißens bei Ferkeln 41-42. Bayrische Landesanstalt für Landwirtschaft, Freising, 95 pp.

Beattie, V.E., Walker, N., Sneddon, I.A., 1996. An investigation of the effect of environmental enrichment and space allowance on the behaviour and production of growing pigs. Applied Animal Behaviour Science (48), 151-158. https://doi.org/10.1016/01681591(96)01031-3.

Benus, R.F., Bohus, B., Koolhaas, J.M., van Oortmerssen, G.A., 1991. Heritable variation for aggression as a reflection of individual coping strategies. Experientia 47 (10), 1008-1019. https://doi.org/10.1007/BF01923336.

Cox, L.N., Cooper, J.J., 2001. Observations on the pre- and post-weaning behaviour of piglets reared in commercial indoor and outdoor environments. Animal Science 72 (01), 75-86. https://doi.org/10.1017/S1357729800055570.

D'Eath, R.B., 2005. Socialising piglets before weaning improves social hierarchy formation when pigs are mixed post-weaning. Applied Animal Behaviour Science 93 (3-4), 199211. https://doi.org/10.1016/j.applanim.2004.11.019.

Ekkel, E.D., van Doorn, C. E. A., Hessing, M.J.C., Tielen, M.J.M., 1995. The Specific-StressFree Housing System Has Positive Effects on Productivity, Health, and Welfare of Pigs. Journal of animal science (73), 1544-1551.

Gentz, M., Lange, A., Zeidler, S., Lambertz, C., Gauly, M., Burfeind, O., Traulsen, I., submitted 2019. Effects of farrowing and rearing systems on tail lesions and losses of docked and undocked pigs. Animal.

Hartigan, J.A., Wong, M.A., 1979. Algorithm AS 136: A K-Means Clustering Algorithm. Applied Statistics 28 (1), 100. https://doi.org/10.2307/2346830.

Herskin, M.S., Thodberg, K., Jensen, H.E., 2015. Effects of tail docking and docking length on neuroanatomical changes in healed tail tips of pigs. Animal 9 (4), 677-681. https://doi.org/10.1017/S1751731114002857.

Hunter, E.J., Jones, T.A., Guise, H. J., Penny, R. H. C. \& Hoste, S., 1999. Tail biting in pigs 1: The prevalence at six UK abattoirs and the relationship of tail biting with docking-, sex and other carcass damage. The Pig Journal - Refereed section 43, 18-32.

Lekic, V., Romanowicz, B., 2011. Tectonic regionalization without a priori information: A cluster analysis of upper mantle tomography. Earth and Planetary Science Letters 308 (12), 151-160. https://doi.org/10.1016/j.epsl.2011.05.050.

Lukashin, A.V., Fuchs, R., 2001. Analysis of temporal gene expression profiles: clustering by simulated annealing and determining the optimal number of clusters. Bioinformatics (17/5), 405-414. 
Maechler, M., Rousseeuw, P., Struyf, A., Hubert, M., Hornik, K., 2012. cluster: Cluster Analysis Basics and Extensions. $\mathrm{R}$ package version 2.1.0.

Meyer-Hamme, S.E.K., Lambertz, C., Gauly, M., 2016. Does group size have an impact on welfare indicators in fattening pigs? Animal 10 (1), 142-149. https://doi.org/10.1017/S1751731115001779.

Moinard, C., Mendl, M., Nicol, C.J., Green, L.E., 2003. A case control study of on-farm risk factors for tail biting in pigs. Applied Animal Behaviour Science (81 (4)), 333-355. https://doi.org/10.1016/S0168-1591(02)00276-9.

Oostindjer, M., van den Brand, H., Kemp, B., Bolhuis, J.E., 2011. Effects of environmental enrichment and loose housing of lactating sows on piglet behaviour before and after weaning. Applied Animal Behaviour Science 134 (1-2), 31-41. https://doi.org/10.1016/j.applanim.2011.06.011.

Paoli, M.A., Lahrmann, H.P., Jensen, T., D'Eath, R.B., 2016. Behavioural differences between weaner pigs with intact and docked tails. Animal Welfare 25 (2), 287-296. https://doi.org/10.7120/09627286.25.2.287.

Parker, M., O'Connor, E., McLeman, M., Demmers, T., Lowe, J., Owen, R., Davey, E., Wathes, C., Abeyesinghe, S., 2010. The impact of chronic environmental stressors on the social behaviour of growing pigs, Sus scrofa. Advances in Animal Biosciences 1 (01), 187. https://doi.org/10.1017/S2040470010003304.

R Core Team, 2016. R: A Language and Environment for Statistical Computing. www.Rproject.org.

Schrøder-Petersen, D.L., Simonsen, H.B., 2001. Tail biting in pigs. Veterinary journal 162 (3), 196-210. https://doi.org/10.1053/tvjl.2001.0605.

Simonsen, H.B., Klinken, L., Bindseil, B., 1991. Histopathologie of intact and docked pig tails. British Veterinary Journal (147), 407-412.

Sparks, R.L., Patton, J., Ganschow, L., 2012. Profiles of more and less successful L2 learners: A cluster analysis study. Learning and Individual Differences 22 (4), 463-472. https://doi.org/10.1016/j.lindif.2012.03.009.

Statham, P., Green, L., Bichard, M., Mendl, M., 2009. Predicting tail-biting from behaviour of pigs prior to outbreaks. Applied Animal Behaviour Science 121 (3-4), 157-164. https://doi.org/10.1016/j.applanim.2009.09.011.

Statham, P., Green, L., Mendl, M., 2011. A longitudinal study of the effects of providing straw at different stages of life on tail-biting and other behaviour in commercially housed pigs. Applied Animal Behaviour Science 134 (3-4), 100-108. https://doi.org/10.1016/j.applanim.2011.08.009.

Taylor, N.R., Main, D.C.J., Mendl, M., Edwards, S.A., 2010. Tail-biting: A new perspective. Veterinary journal 186 (2), 137-147. https://doi.org/10.1016/j.tvjl.2009.08.028. 
Taylor, N.R., Parker, R.M.A., Mendl M., Edwards, S.A., Main, D.C.J., 2012. Prevalence of risk factors for tail biting on commercial farms and intervention strategies. The Veterinary Journal, 77-83. https://doi.org/10.1016/j.tvjl.2012.03.004.

Valros, A., 2018. Tail biting, in: Špinka, M. (Ed.), Advances in pig welfare. Woodhead Publishing an imprint of Elsevier, Duxford, United Kingdom, 137-166.

van Putten, G., 1969. An Investigation into Tail-Biting among Fattening Pigs. British Veterinary Journal 125 (10), 511-517. https://doi.org/10.1016/S0007-1935(17)48710-0. Zason Chian, L.K., John Wang, C.K., 2008. Motivational Profiles of Junior College Athletes: A Cluster Analysis. Journal of Applied Sport Psychology 20 (2), 137-156. https://doi.org/10.1080/10413200701805265.

Zonderland, J.J., Bracke, M.B.M., den Hartog, L.A., Kemp, B., Spoolder, H.A.M., 2010. Gender effects on tail damage development in single- or mixed-sex groups of weaned piglets. Livestock Science 129 (1-3), 151-158.

https://doi.org/10.1016/j.livsci.2010.01.018. 


\title{
PAPER THREE
}

\section{Different housing systems during nursery: tail lesions and losses, skin lesions and performance during rearing and fattening period}

\author{
M. Gentz ${ }^{1}$, A. Lange ${ }^{1}$, O. Burfeind ${ }^{2}$ and I. Traulsen ${ }^{1}$ \\ ${ }^{1}$ Livestock Systems, Department of Animal Sciences, Georg-August-University, \\ Albrecht-Thaer-Weg 3, 37075 Göttingen, Germany \\ ${ }^{2}$ Chamber of Agriculture of Schleswig-Holstein, Gutshof 1, 24327 Blekendorf, \\ Germany
}

Corresponding author: M. Gentz (maria.gentz@uni-goettingen.de) 


\section{Implications}

Artificial rearing during the nursery period gained more importance, because the litters of modern pig farming exceed the number of functional teats of the sow. Therefore, some piglets are reared artificially. This type of housing has a positive effect on tail lesions and losses as well as skin lesions through early socialisation and stable groups. Moreover, AR pigs showed comparable daily weight gains but for practical recommendations concerns of reduced animal welfare and economical aspects must be included in critical reflection.

\section{Abstract}

Artificial rearing is one method for pig farmers to raise large litters which outnumber the functional teats of the sow. Litter size increased during the last years and natural nurses to raise the piglets are not always available. Artificial rearing involves early weaning of piglets from their mothers two days post partum to be reared in specialised housing systems. The current study aims to investigate long-term effects of artificial rearing and reduced regrouping during nursery period on tail biting and development of pigs. Therefore, a comparison of artificially reared $(A R, N=163)$ and piglets raised in a conventional farrowing crate $(F C, N=264)$ during the nursery period was conducted. Half of the pigs were undocked. The investigation was focussed on tail lesions and losses, skin lesions and performance traits. Regarding the results of the tail lesions the AR pigs showed fewer lesions than the FC pigs. Less than $15 \%$ of the AR pigs had tail lesions at all. In general, tail lesions of docked pigs were on a lower level than of undocked pigs. Tail losses of undocked pigs at the end of rearing were not significantly different. At the end of fattening period only $6.9 \%$ of the AR pigs lost parts of their tails differing significantly from the percentage of FC pigs with tail loss $(60.2 \%)$. The AR piglets $(21.7 \%)$ showed significantly fewer skin lesions than FC pigs $(80.2 \%)$ after rehousing for rearing. After regrouping and rehousing for fattening, more than $94 \%$ of the pigs from the two housing systems during nursery showed skin lesions. The daily weight gain of FC piglets $(256.0 \mathrm{~g})$ during nursery period was significantly higher than the values of AR pigs. During rearing AR pigs had the highest daily weight gain (461.6 g). There was no significant difference during fattening period. The carcass traits were not significantly different either. Artificial rearing has a positive effect on tail lesions and losses as well as skin lesions through early socialisation and stable groups. Moreover, AR pigs showed comparable daily weight gains but for practical recommendations concerns of reduced animal welfare and economical aspects must be included in critical reflection.

Keywords: Artificial rearing; piglets; nursery period; tail biting; reduced regrouping 


\section{Introduction}

During the last few years breeding has led to a large litter size (Rutherford et al., 2013; Tomiyama et al., 2011). Piglets which were born in large litters can have a huge weight variance whereby the pre-weaning survival rate for the lighter animals is often reduced (Andersen et al., 2011; Milligan et al., 2002; Wolf et al., 2008). In addition, the competition at the teats is increased and the number of live born piglets may outnumber the availability of functional teats (Andersen et al., 2011; Milligan et al., 2001). To raise a large number of piglets in commercial pig farms often nurse sows or artificial piglet rearing systems can be found (Baxter et al., 2013). These nurses raise the piglets after colostrum intake at their mother until they would have been weaned from their biological mother. After colostrum intake (two days post partum) the piglets that cannot be raised by the sow are moved to the nurse (Baxter et al., 2013). If there is no natural nurse, the animals are housed into an artificial rearing system. Removing piglets from their mother at an early stage affects their animal welfare (Rzezniczek et al., 2015). Schmitt et al. (2019) showed the detrimental effects of artificial rearing on piglets' behaviour, growth and welfare. Due to a lack of appropriate stimuli for accommodating the behavioural needs of the young piglets, they tend to perform abnormal behaviour (Widowski et al., 2005). In the artificial housing systems, the piglets get milk replacer, pre-starter feeding and water ad libitum, but piglets have also the need to suckle. This need is often not fulfilled in artificial nurseries (Widowski et al., 2005). The milk is usually taken from a nipple drinker with an open liquid level. Widowski et al. (2005) showed that feeding systems that accommodates both suckling and massage can significantly reduce piglet-directed behaviour and may facilitate social housing of artificially reared piglets (Widowski et al., 2005).

Previous research mainly focussed on pre-weaning behaviour of artificially reared pigs (Rzezniczek et al., 2015; Schmitt et al., 2019). Rzezniczek et al. (2015) found out that the wellknown signs of stress after weaning also occur in this early stage of life. The piglets showed vocalisations, development of abnormal behaviours and growth impairments (Orgeur et al., 2001). One of the typical reactions of pigs to stress is abnormal behaviour such as tail biting (Weary et al., 2008). Schmitt et al. (2019) detected more agonistic interactions as well as oral manipulative behaviours (ears, tails, belly nosing) during nursery period. Belly-nosing during suckling period for instance is rarely observed in sow-reared piglets whereas the occurrence is higher in early-weaned piglets (Weary et al., 1999; Worobec et al., 1999). This abnormal behaviour is considered as a substitute for the natural suckling (Widowski et al., 2008). As there is no sow, the piglets show these behaviours to each other (Widowski et al., 2005). Apart from early weaning stress, artificially reared pigs are housed in lower space allowance (about $1 \mathrm{~m}^{2}$ ) than piglets from other farrowing systems (about $3.6 \mathrm{~m}^{2}$ ) (Baxter et al., 2013). This also influences the behaviour of the animals. 
Regarding the performance Cabrera et al. (2010) and van Beirendonck et al. (2015) found heavier weaning weights in artificially reared piglets compared to sow-reared piglets but in later development, the higher weights could not be maintained (Cabrera et al., 2010). Cabrera et al. (2010) also measured a lower carcass quality at slaughter of artificially reared piglets. However, the early forming of a group and keeping it stable over time also has advantages: The positive effect of early socialisation of piglets during suckling before weaning also includes long-term benefits of the social behaviour (D'Eath, 2005). After regrouping these pigs built a new hierarchy more quickly. This can affect agonistic behaviour as well as tail biting in a positive way (Coutellier et al., 2007; D’Eath, 2005).

The aim of the study was to find out whether artificial rearing during nursery period can have effects on the pigs particularly regarding tail biting and their long-term development. $A$ comparison of different housing systems during nursery period including artificially reared pigs and conventional farrowing crate pigs concerning tail lesions and losses, skin lesions, daily weight gain and carcass traits was conducted.

\section{Animals, Material and Methods}

The dataset comprised of 427 rearing pigs and 157 fattening pigs. The data was acquired from Mai 2017 to August 2018 at the research farm Futterkamp at the chamber of Agriculture of Schleswig-Holstein located in northern Germany. Within five batches, cross-breeds (Pietrain $x$ (Large White $x$ Landrace)) were housed docked (224 pigs) and undocked (203 pigs) (batch 1, 2 and 4 docked animals; batch 3 and 5 undocked animals). Males were raised as intact males. During nursery period (from farrowing until 27th day of life) the piglets were kept in two different housing systems: (1) artificial rearing $(\mathrm{AR}, \mathrm{N}=163)$ and (2) conventional farrowing crate (FC, $\mathrm{N}=264)$.

The AR animals were weaned from their mothers two days post partum (after colostrum intake) and housed into an artificial rearing system in groups of 12 piglets $\left(0.1 \mathrm{~m}^{2} / \mathrm{pig}\right)$. These groups (artificial litters) contained piglets from three to seven natural litters. The pen where the piglets spent the first two weeks of the nursery period was a plastic box on stilts. The piglets had a heating lamp. Furthermore, there was a trough with pre-starter piglet feed and two nipple drinkers with an open liquid level where the animals were provided milk replacer ad libitum. Additionally, they had a water nipple drinker. After two weeks in the artificial rearing boxes, they were transferred to a pen at ground level which offered a higher space allowance $(0.29$ $\mathrm{m}^{2} /$ pig) and mash feed. A heating lamp was offered as well. In the FC, the sows farrowed in a conventional farrowing crate pen $\left(5.2 \mathrm{~m}^{2}\right)$, where they were fixed permanently until weaning of the piglets. The piglets had a piglet nest with a heating lamp and they got pre-starter piglet feed, as well. 
The average litter size was 16.1 live born piglets per sow. As the litter size was adjusted to 14 piglets per sow according to the number of functional teats, an average of two piglets per sow were weaned two days post partum and rehoused to artificial rearing. According to regular management practices the heaviest piglets from the litters were transferred to the artificial rearing system. Consequently, this was an observational study.

After 27 days of suckling the FC piglets were weaned, and FC and AR piglets were rehoused and regrouped to a conventional rearing system $\left(0.44 \mathrm{~m}^{2} / \mathrm{pig}\right) .13$ pigs were housed per pen and mixed within their housing system during nursery period (no mixing of AR and FC pigs) (Figure 1). The FC pigs were born in three to seven litters, consequently they knew at least one of their new group members because it was one of the siblings. The AR pigs were only rehoused and one pig from another AR-pen was added to create comparable group sizes. The rearing pens had plastic flooring and feeding troughs with twelve feeding spaces. The pigs were housed mixed-sex.

After 40 days of rearing, the pigs were regrouped and rehoused for fattening $\left(0.89 \mathrm{~m}^{2} / \mathrm{pig}\right)$ and sorted by sex. During fattening they were kept in groups of 14 animals. The AR and FC groups were built of two rearing pens (26 pigs) so twelve rearing pigs left the study. Due to the regrouping, both $A R$ and FC pigs were exposed to the challenges of a new group. The fattening pens had concrete flooring and feeding troughs with twelve feeding spaces, as well. The pigs were fed ad libitum with conventional dry feed adjusted to their age. With reaching $120 \mathrm{~kg}$ live weight the animals were transported to slaughter. During their whole life, the pigs always had access to at least one or several different enrichment materials, for example, pieces of wood, ropes, jute bags or troughs filled with dried peas and grass pellets. If an outbreak of tail biting occurred, pigs were offered additional or renewed enrichment material. Both groups were treated the same. 


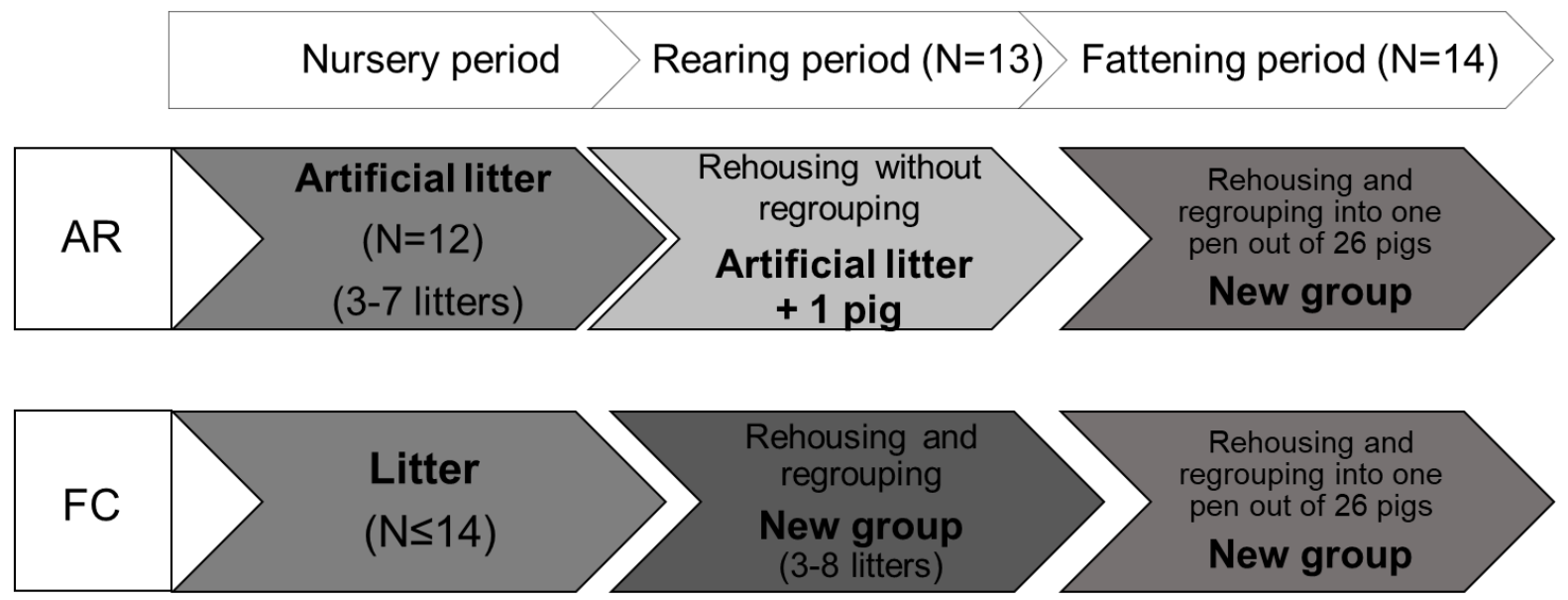

Figure 1. Experimental design $(\mathrm{N}=$ number of pigs per pen, $A R=$ artificial rearing, $F C=$ farrowing crate).

All animals were housed in accordance with EU (European Directive 2008/120/EC) and national law (Animal Welfare Act (18/05/2016), Animal Welfare and Animal Husbandry Ordinance (05/02/2014).

\subsection{Recorded traits}

\section{Tail lesions and losses}

The scoring of tail lesions and losses started one day after rehousing for rearing pens. The piglets' tails were scored individually once a week starting at 28 days after birth for 18 assessment weeks using a modified Schwarzenauer key (Abriel and Jais, 2013). The assessor entered the pen. If there were no visible lesions the tail was scored "0". A tail "lesion" means that the tail has slight scratches or bite marks (score 1), a "severe lesion" shows deeper, flat lesions (score 2) and a "very severe lesion" would be a deep flat lesion which is greater than $2 \mathrm{~cm}$ (score 3). For the statistical analysis, the tail lesion score was summarised due to the low occurrence (<2\%) of severe lesions into "no tail lesions" (0) and "tail lesions" (1). Tail losses were divided into quarters of the tail, which resulted in score 0 (intact tail) to score 4 (100\% lost). These scores were also summarised into "intact tail" and "tail loss" due to very low occurrence $(<5 \%)$ of losses of more than one quarter.

\section{Skin lesions}

One day after rehousing for rearing the assessment of skin lesions started. During rearing, the skin lesion scoring took place biweekly, during fattening period the animals were scored every fourth week. Additionally, the pigs were scored the week before and after rehousing for fattening. This resulted in a skin lesion scoring in weeks 1, 3, 5, 6 (rearing) and weeks 7, 11, 15 (fattening). The pigs were scored following the Welfare Quality ${ }^{\circledR}$ assessment protocol 
applied to growing and finishing pigs (Welfare Quality®, 2009). The assessment was carried out by three assessors, who had completed an internal training and observer cross check. For each assessment at least two of the three persons were present. The assessor scored the animal's front on the right side from approximately $0.5 \mathrm{~m}$. The scores were grounded on the number of lesions. A scratch longer than $2 \mathrm{~cm}$ or two parallel scratches with only $0.5 \mathrm{~cm}$ between them or a small wound with a diameter of maximum $2 \mathrm{~cm}$ was considered "one lesion". A bleeding wound between 2 and $5 \mathrm{~cm}$ or a healed wound greater than $5 \mathrm{~cm}$ was considered "five lesions" and a deep open wound greater than $5 \mathrm{~cm}$ was considered "16 lesions". The skin lesion score ranged from A to C: according to the number of lesions, the body part was scored for no or up to 4 lesions (minor lesions, score A), for 5 to 10 lesions (moderate lesions, score B) and when more than 10 lesions were visible (severe LS, score $\mathrm{C}$ ). In this study only the skin lesions of the front body have been considered (Welfare Quality $\AA$, 2009). For statistical analysis the scores were summarised into a binary trait, (0 (no lesions) and 1 (skin lesions)) because of the very low occurrence $(<5 \%)$ of severe lesions.

\section{Performance traits}

The staff of the farm recorded the birth weight, the weight at 27 days of age, the 40-days weight and the sales weight. The animals were weighed individually (identification via individual ear tags). The absolute weights were converted into daily weight gains (g/day) per period for analysis. All animals were slaughtered in the same slaughterhouse in Northern Germany. The Auto FOM III method was applied (Choi et al., 2018). The carcass weight and the weights of all cuts per animal were recorded. For further analysis the slaughter weight, the lean meat percentage and the ham weight were used as a selection of the most relevant carcass parameters (Weatherup et al., 1998).

\subsection{Statistical analysis}

The data analysis was conducted with SAS® 9.4 (SAS Institute Inc., USA). The binomial data as tail lesions, tail losses and skin lesions were analysed by GLIMMIX procedure. The MIXED procedure was used for data for where a normal distribution could be assumed (performance traits: daily weight gain and carcass traits). For each parameter the model with the lowest values for 'Akaike's information criterion corrected' AICC (Hurvich and Tsai, 1989) and 'Bayesian information criterion' BIC (Schwarz, 1978) was used for further evaluation. The significance of differences in the least square means for each model was adjusted with the Bonferroni-correction to correct for multiple comparisons. For the models the fixed effects housing system during nursery period (AR, FC), docking status (undocked, docked) and sex (male, female) were examined. 
In the GLIMMIX models based on scoring data (tail lesions, tail losses and skin lesions), the interaction batch*pen was used as a random effect. The model of the tail lesions was calculated over all 18 assessment weeks to show the development of the lesions. Consequently, an additional effect of assessment week (1-18) and assessment week nested within the interaction between farrowing system and docking status was added to the model. The tail loss models were calculated for the end respectively of rearing and fattening period. Moreover, the analysis was conducted for undocked pigs due to nearly no occurrence of tail losses for docked pigs. Therefore, the docking status as fixed effect was dropped.

There were two models for the skin lesions. One contained the day after rehousing for rearing and one was calculated for the day after regrouping and rehousing for fattening.

The analysis of daily weight gains was carried out in stages, so the periods of nursery, rearing and fattening were analysed separately. The batch and respectively the sow (nursery period) or respectively the pen (rearing and fattening period) were used as random effects. The weight at the beginning of the calculated period was used as a covariable to take the different initial weights of the respective animals into account.

For the models of carcass traits, the batch and the pig were used as random effects.

\section{Results}

The results show that each used fixed effect was affecting the tested traits significantly $(p \leq 0.05)$ within a specific model (Table 1). 
Table 1. Statistical significances for the fixed effects of the examined traits ( ${ }^{*} p \leq 0.05 ; N=$ number of pigs).

\begin{tabular}{|c|c|c|c|c|c|c|c|c|c|c|c|}
\hline & \multicolumn{3}{|c|}{ Tail } & \multicolumn{2}{|c|}{ Skin } & \multicolumn{6}{|c|}{ Performance traits } \\
\hline & \multirow{2}{*}{$\begin{array}{c}\text { Lesions } \\
\begin{array}{r}\text { Assessment } \\
\text { weeks 1-18 }\end{array}\end{array}$} & \multicolumn{2}{|c|}{$\begin{array}{c}\text { Losses } \\
\text { (undocked pigs) }\end{array}$} & \multicolumn{2}{|c|}{ Lesions } & \multicolumn{3}{|c|}{ Daily weight gain } & \multicolumn{3}{|c|}{ Carcass traits } \\
\hline & & $\begin{array}{l}\text { End of } \\
\text { rearing }\end{array}$ & $\begin{array}{r}\text { End of } \\
\text { fattening }\end{array}$ & $\begin{array}{c}\text { Day after } \\
\text { rehousing } \\
\text { for rearing }\end{array}$ & $\begin{array}{r}\text { Day after } \\
\text { regrouping } \\
\text { for fattening }\end{array}$ & $\begin{array}{r}\text { Nursery } \\
\text { period }\end{array}$ & $\begin{array}{c}\text { Rearing } \\
\text { period }\end{array}$ & $\begin{array}{r}\text { Fattening } \\
\text { period }\end{array}$ & $\begin{array}{r}\text { Slaughter } \\
\text { weight }\end{array}$ & $\begin{array}{r}\text { Lean meat } \\
\text { percentage }\end{array}$ & $\begin{array}{r}\text { Ham } \\
\text { weight }\end{array}$ \\
\hline $\mathrm{N}$ & 427 & 194 & 99 & 427 & 157 & 340 & 338 & 143 & 108 & 108 & 108 \\
\hline $\begin{array}{l}\text { Housing system } \\
\text { during nursery } \\
\text { period }\end{array}$ & 0.71 & 0.99 & $0.02^{*}$ & $<0.01^{*}$ & 0.58 & $<0.01^{*}$ & $<0.01^{*}$ & 0.81 & 0.32 & 0.17 & 0.16 \\
\hline Docking status & 0.92 & $--^{1}$ & $--^{1}$ & 0.52 & 0.85 & 0.28 & 0.74 & $0.05^{*}$ & 0.48 & 0.13 & 0.18 \\
\hline Sex & 0.44 & 0.08 & 0.60 & 0.09 & 0.93 & 0.42 & 0.17 & 0.47 & 0.39 & 0.37 & 0.55 \\
\hline Assessment week & $<0.01^{*}$ & $--^{1}$ & $--^{1}$ & $--^{1}$ & $--^{1}$ & $--^{1}$ & $--^{1}$ & $--^{1}$ & $--^{1}$ & $--^{1}$ & $--^{1}$ \\
\hline $\begin{array}{l}\text { Assessment week } \\
\text { (Housing system } \\
\text { during nursery } \\
\text { period * Docking } \\
\text { status) }\end{array}$ & $<0.01^{*}$ & $--^{1}$ & $--^{1}$ & $--^{1}$ & $--^{1}$ & $--^{1}$ & $--^{1}$ & $--^{1}$ & $--^{1}$ & $--^{1}$ & --1 \\
\hline
\end{tabular}

\footnotetext{
${ }^{1}$ not included in the final model.
} 


\subsection{Tail lesions}

A significant effect of the assessment week nested within the interaction between farrowing system and docking status and assessment week was observed $(p<0.05)$, while the effect of the housing system during nursery period, docking status and sex was not significant $(p>0.05)$ (Table 1).

Regarding the back transformed LS-means of the tail lesions of the undocked pigs during 18 assessment weeks after rehousing for rearing, the AR animals showed fewer lesions than the FC animals (Figure 2). Most of the assessment weeks the level of AR pigs with tail lesions stayed under $15 \%$. Starting in the third week the undocked FC pigs showed a persistently high level of tail lesions until week 15 (about 25\%). The biting of the docked FC pigs is shifted backwards. Starting in week seven it shows a falling tendency after week 13 . The level of tail lesions of docked pigs was much lower than the level of undocked pigs.

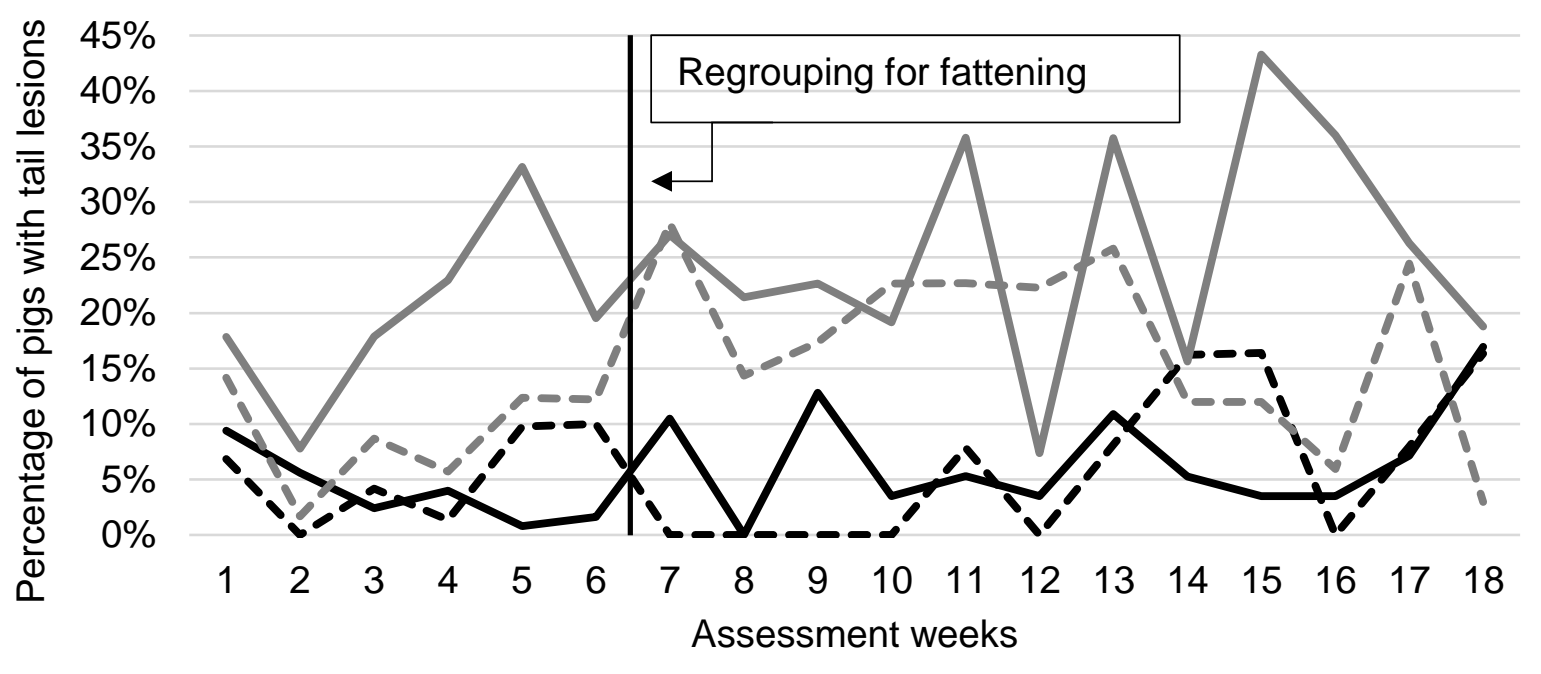

—AR undocked -- AR docked $\longrightarrow$ FC undocked -- FC docked

Figure 2. Frequency of back transformed tail lesion estimates during of the rearing and fattening period for docked and undocked animals per housing system during nursery period ( $\mathrm{AR}=$ artificial rearing, $\mathrm{FC}=$ farrowing crate) .

\subsection{Tail losses}

The tail losses of the undocked pigs at the end of rearing were not significantly different although losses were numerically different. No AR pigs suffered any tail loss during rearing period but $11.81 \%$ of the FC pigs lost a part of their tails (Figure 3). At the end of fattening the housing systems during nursery period affected tail losses significantly, $6.89 \%$ of the AR pigs lost parts of their tails. This value was significantly different from the percentage of pigs with tail loss from the FC (60.23\%). 


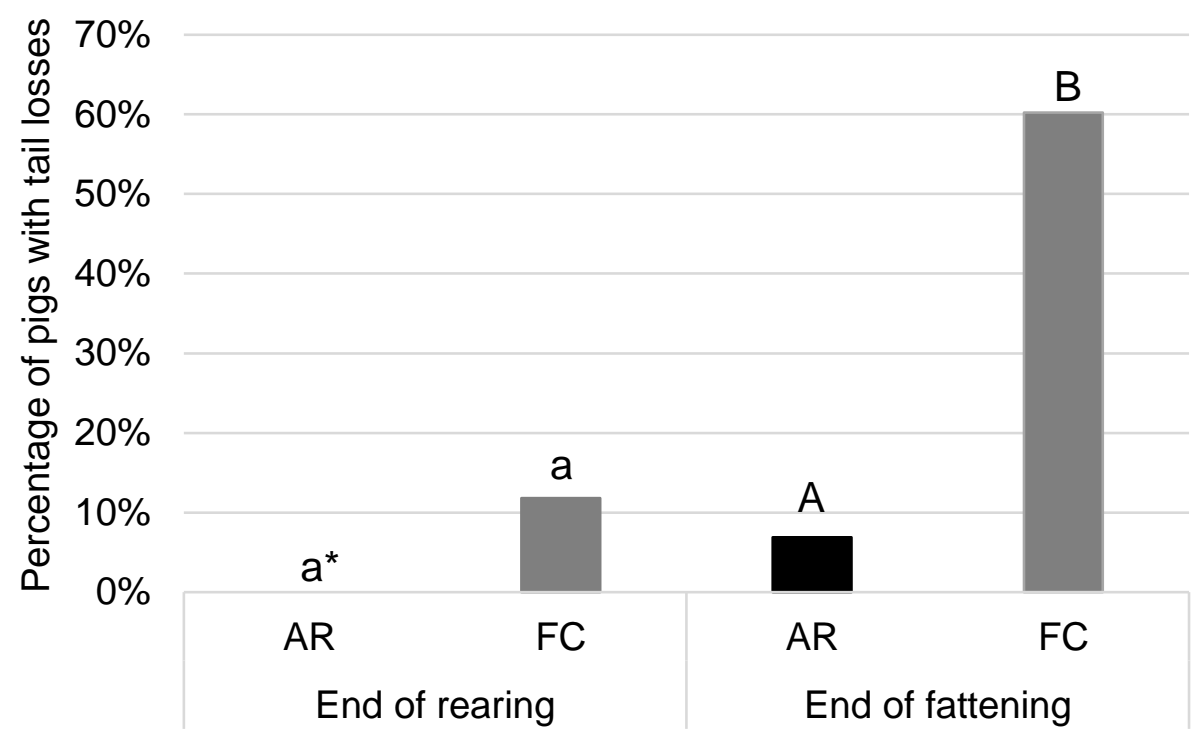

Figure 3. Frequency of back transformed tail loss estimates for end of rearing and end of fattening (undocked pigs), ( $\mathrm{AR}=$ artificial rearing, $\mathrm{FC}=$ farrowing crate)

$a-b, A-B$ : Significant differences between housing systems during nursery period $(p \leq 0.05)$. ${ }^{*} A R$ had no tail losses during rearing period.

\subsection{Skin lesions}

The model of the skin lesions showed significant effects of the housing systems during nursery period for the day after rehousing for rearing but the model for the day after regrouping for fattening did not show significant effects. The AR animals $(21.70 \%)$ had significantly fewer skin lesions than FC animals (80.20\%) (Figure 4). The effect of the housing systems during nursery period was apparently no longer present after regrouping and rehousing for fattening. The housing systems during nursery period did not show significant differences during the day after regrouping to fattening ( $A R=94.11 \%, F C=96.28 \%)$. 


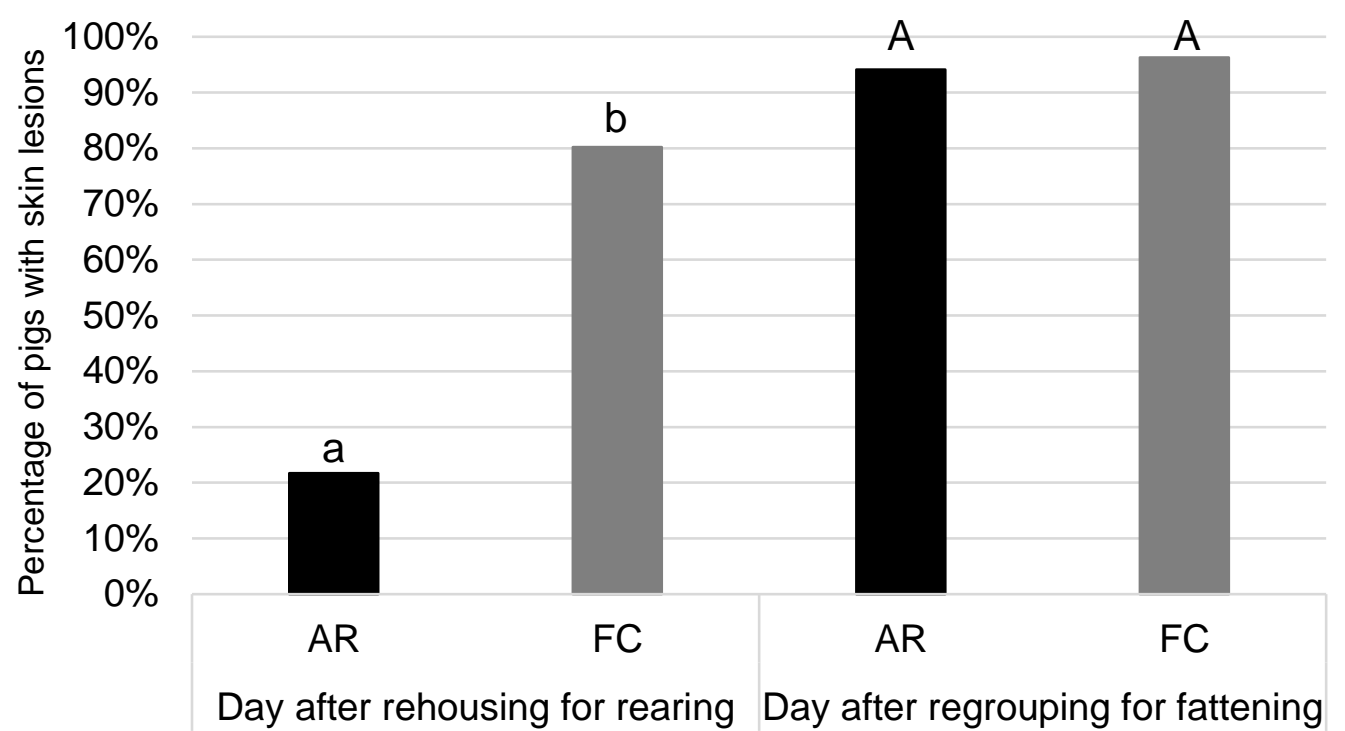

Figure 4. Frequency of back transformed skin lesion estimates for the day after rehousing for rearing and the day after regrouping for fattening $(A R=$ artificial rearing, $F C=$ farrowing crate) $a-b, A-B$ : Significant differences between housing systems during nursery period respectively docking status $(p \leq 0.05)$ within point of time.

\subsection{Performance}

The models for daily weight gain during nursery, rearing and fattening period showed a significant effect of the housing system during nursery period and rearing period, but there was no difference during fattening $(p<0.05)$. The daily weight gain of the docked and undocked pigs only differed during fattening period. The sex had no significant effect (Table 1).

Regarding the individual differences of the housing system during nursery during nursery period the AR piglets' (136.4 g) daily weight gain was only half as high as the weight gain of the FC $(256.0 \mathrm{~g})$ piglets (Table 2$)$. During rearing period, the daily weight gain of the AR piglets $(461.6 \mathrm{~g})$ was significantly higher than the values of the FC piglets $(405.6 \mathrm{~g})$. There was no significant difference of the housing system during nursery during fattening period ( $A R=920.4$ $\mathrm{g}, \mathrm{FC}=927.8 \mathrm{~g})$. The significant difference between undocked $(890.5 \mathrm{~g})$ and docked pigs (957.6 g) was only found during fattening (Table 2). There were no significant differences in the nursery and rearing period.

The models of the carcasses showed no significant differences for the housing system during nursery and the sexes ( $p>0.05)$ (Table 1). The docking status had no significant influence, as well $(p>0.05)$. 
Table 2. Least square means (LSM) and standard error (SE) of the farrowing systems and the docking status in performance models (daily weight gain in $\mathrm{g}$ ) of pigs during nursery, rearing and fattening period as well as slaughter weight $(\mathrm{kg})$, lean meat percentage (\%) and ham weight $(\mathrm{kg})$.

\begin{tabular}{lcc|cc}
\hline & \multicolumn{2}{c}{ Farrowing system } & \multicolumn{2}{c}{ Docking status } \\
\hline AR & FC & Undocked & Docked \\
\hline Daily weight gain & & & & \\
\hline Nursery period & $136.4^{\mathrm{a}}(6.2)$ & $256.0^{\mathrm{b}}(5.0)$ & $200.9^{\mathrm{a}}(6.8)$ & $191.5^{\mathrm{a}}(6.0)$ \\
Rearing period & $461.6^{\mathrm{a}}(30.0)$ & $405.6^{\mathrm{b}}(28.9)$ & $430.1^{\mathrm{a}}(31.2)$ & $437.1^{\mathrm{a}}(30.3)$ \\
Fattening period & $920.4^{\mathrm{a}}(27.1)$ & $927.8^{\mathrm{a}}(20.8)$ & $890.5^{\mathrm{a}}(24.0)$ & $957.6^{\mathrm{b}}(23.3)$ \\
\hline Carcass traits & & & & \\
\hline Slaughter weight & $98.78^{\mathrm{a}}(0.90)$ & $100.86^{\mathrm{a}}(0.45)$ & $100.39^{\mathrm{a}}(0.68)$ & $99.24^{\mathrm{a}}(0.87)$ \\
Lean meat & $56.79^{\mathrm{a}}(0.59)$ & $59.54^{\mathrm{a}}(0.37)$ & $59.91^{\mathrm{a}}(0.45)$ & $56.79^{\mathrm{a}}(0.57)$ \\
Ham weight & $18.62^{\mathrm{a}}(0.21)$ & $19.62^{\mathrm{a}}(0.16)$ & $19.55^{\mathrm{a}}(0.16)$ & $18.70^{\mathrm{a}}(0.20)$ \\
\hline
\end{tabular}

a-b: Significant differences between housing systems during nursery period respectively docking status $(p \leq 0.05)$ within trait.

\section{Discussion}

\subsection{Stress}

Regarding the prevalence of tail lesions in the present study, the level was much lower at AR pigs than at FC pigs. The number of pigs with tail lesions was increasing for FC pigs three weeks after rehousing for rearing and the high level stayed until week 15 . Tail biting is a compensatory behaviour of pigs to reduce the stress level (Rushen, 1993; Schrøder-Petersen and Simonsen, 2001). Weaning causes stress due to the separation from the mother, regrouping and new environment (Weary et al., 2008). The coping hypothesis proposes that stereotypies reduce the physiological stress reaction linked to environmental impacts (Rushen, 1993). In the current study the assessment of tail lesions started at rearing period. At this point FC pigs were weaned and rehoused, but the AR pigs were only rehoused. The separation from the sow and the change of environment occurred already two days post partum for AR pigs and therefore AR piglets were exposed to early weaning stress according to McGlone and Curtis (1985) and Smith et al. (2010). Rzezniczek et al. (2015) also assessed signs of early weaning stress at AR piglets. The current study could not find increased tail biting as a behavioural abnormality at the AR pigs because the evaluation started after nursery period, still they started at lower tail lesion levels than FC piglets in assessment week 1. The FC pigs on the other hand were exhibiting the typical tail biting. Due to the accumulation of stress, they start to bite in the middle of the rearing period, which can be also found in literature (Veit et al., 2016). According to the literature, early weaning stress leads to behavioural disorders of $A R$ 
pigs during nursery period (Schmitt et al., 2019; Weary et al., 1999; Worobec et al., 1999). In the current study behavioural observations weren't available for this early stage of life.

\subsection{Reduced regrouping}

It is remarkable that pigs from AR had a lower level of tail lesions. This can probably be attributed to the fact that the group has been kept stable during nursery and rearing period (Coutellier et al., 2007). The piglets were grouped two days post partum and stayed in this group until regrouping for fattening. So, although the AR pigs have been rehoused twice more often (during nursery period) than FC pigs, they had fewer tail lesions. The positive effect of reduced stress by early grouping and rehousing in constant groups is already known (Coutellier et al., 2007) and can be transferred to the situation in AR of the present study.

The conditions after rehousing for rearing were very different for the piglets. The AR pigs were only rehoused but remained in their familiar group (joined by one additional animal). The FC pigs in contrast just knew their littermates up to rehousing for rearing. Besides to regrouping the FC pigs were separated from the mother and rehoused to a new environment. The regrouping and rehousing after weaning can lead to stress and this can cause aggressive behaviour (Weary et al., 2008). The formation of a hierarchy requires fights of rank and this could lead to skin lesions (McGlone and Curtis, 1985). However, the number and intensity of the ranking fights can vary, depending on the degree of socialisation and the age of the animals. A stable socialisation can reduce the number and intensity of fights (D'Eath, 2005). In the current study the consequences of reduced regrouping can be seen at AR pigs, which show fewer skin lesions than FC pigs after rehousing for rearing. Behaviour analysis would have been beneficial to evaluate a possible impact of the housing system during nursery here. The effect of housing system during nursery was not significant after regrouping to fattening. In contrast to rehousing for rearing, at this time all pigs were regrouped (new groups of 14 out of 26 rearing pigs) and rehoused. This led consequently to rank fights and the formation of new hierarchies. Nearly all pigs were affected by skin lesions (>94\%).

\subsection{Feeding}

Changes in feeding can also lead to stress reactions and thus to tail biting (Taylor et al., 2012). As usual, the feeding was adapted to the needs of the animals during rearing, at the beginning of fattening and towards the end of fattening. The number undocked AR animals with tail lesions increased slightly in week 7 as well as the levels of tail lesions of FC pigs raised in week 7 . The changes in feeding during week 14 appear to have affected the undocked FC pigs. 
The daily weight gains of the pigs from the two housing systems during nursery differed significantly during nursery and rearing period. AR piglets did not drink in a natural way, but they had nipple drinkers. This unnatural way of drinking can lead to a reduced feed intake and thus cause a losses in weight gain (Widowski et al., 2005). Furthermore, AR piglets often suffer from severe diarrhoea (Vos et al., 2014). An increased incidence of diarrhoea was also observed in the current study. This means that they cannot effectively absorb the energy in the feed and this results in lower weight gains (Vos et al., 2014).

Feeding was always carried out in the same way during rearing and fattening period. According to comparable results in the daily gains, there were no significant differences between the housing systems during nursery period in terms of carcass weight, lean meat and ham weight.

\subsection{Sexual Maturity}

Regarding the tail losses at the end of fattening period there was no differences regarding the sexes. In literature, several authors found differences (Statham et al., 2011; Zonderland, 2010). The onset of sexual maturity resulted in animals that became more restless due to hormonal and behavioural changes (Berry and Signoret, 1984). Furthermore, the sex did not affect the skin lesions which can also be found in other studies (Meese and Ewbank, 1973; Scollo et al., 2013). Expected differences in sex in daily gains or carcass traits could not be seen in the current study, neither. Previous findings from literature showed that boars have higher gains and sows tend to fatten (Weatherup et al., 1998).

\subsection{Animal welfare}

Apart from all the positive and negative aspects of artificial rearing, it must be considered that this type of animal housing is not recommended from an animal welfare point of view. Separation from the sow at such an early stage is ethically questionable. Natural nurses should be preferred for the piglets that cannot be raised by their mother. However, this is not always possible, as there are not always slaughter sows available that are used for this purpose. In addition, the natural nurses do not have any colostrum and therefore weaning should not happen as two days post partum. There is nevertheless early weaning stress, although the environment is comparable, and the milk intake can also take place naturally (Cabrera et al., 2010; van Beirendonck et al., 2015). The economic aspect of artificial rearing should also be considered in future studies. The care and the feeding of the piglets is expensive. Overall this includes the question whether a decrease or at least constant litter size should be the current aim. 


\section{Conclusion}

Despite concerns on animal welfare, the results show that the AR pigs did not exhibit any negative conspicuous features on the analysed traits compared to the other two farrowing systems. They have a lower level of tail lesions and they suffer fewer tail losses. Additionally, they have fewer skin lesions after rehousing for rearing. The daily weight gain during fattening period was comparable to the values of FC and the carcass did not show remarkable differences. Nevertheless, the positive effect of the housing system cannot be separated from the reduced regrouping effect in the present study. Fewer regroupings and therefore less stress due to no formation of new hierarchies contribute well to less abnormal behaviour.

Author Contributions: Maria Gentz: Conceptualisation, Methodology, Software, Validation, Formal analysis, Investigation, Data curation, Writing original draft, Writing - review and editing, Visualization. Anita Lange: Methodology, Software, Validation, Investigation, Data curation, Writing - review and editing. Onno Burfeind: Validation, Investigation, Resources, Writing review and editing, Project administration, Funding acquisition. Imke Traulsen: Conceptualisation, Methodology, Validation, Resources, Writing - review and editing, Supervision, Project administration, Funding acquisition. All authors have read and agreed to the published version of the manuscript.

Acknowledgements: This research was funded by the Federal Office for Agriculture and Food of Germany and the Landwirtschaftliche Rentenbank (project no.: 2817205413; 758914) and by the H. Wilhelm Schaumann Foundation.

Ethics statement: All the animals were housed in accordance with EU (European Directive 2008/120/EC) and national law (Animal Welfare Act (18/05/2016), Animal Welfare and Animal Husbandry Ordinance (05/02/2014).

Declaration of interest: The authors declare no conflict of interest. The funders did not play a role in the design of the study, in the collection, analyses, or interpretation of data, in the writing of the manuscript, or in the decision to publish the results. 


\section{References}

Abriel, M., Jais, C., 2013. Mehr Tierwohl - Maßnahmen im Bereich der Haltung: Versuche zur Reduzierung des Schwanzbeißens bei Ferkeln 11, 41-42. Bayrische Landesanstalt für Landwirtschaft, Freising, 95 pp.

Andersen, I.L., Nævdal, E., Bøe, K.E., 2011. Maternal investment, sibling competition, and offspring survival with increasing litter size and parity in pigs (Sus scrofa). Behavioral ecology and sociobiology 65 (6), 1159-1167. https://doi.org/10.1007/s00265-010-1128-4.

Baxter, E.M., Rutherford, K.M.D., D'Eath, R.B., Arnott, G., Turner, S.P., Sandøe, P., Moustsen, V.A., Thorup, F., Edwards, S.A., Lawrence, A.B., 2013. The welfare implications of large litter size in the domestic pig II: management factors. Animal Welfare 22 (2), 219-238. https://doi.org/10.7120/09627286.22.2.219.

Berry, M., Signoret, J.-P., 1984. Sex play and behavioural sexualization in the pig. Reproduction Nutrition Dévelopment (24(5A)), 507-513.

Cabrera, R.A., Boyd, R.D., Jungst, S.B., Wilson, E.R., Johnston, M.E., Vignes, J.L., Odle, J., 2010. Impact of lactation length and piglet weaning weight on long-term growth and viability of progeny. Journal of animal science 88 (7), 2265-2276. https://doi.org/10.2527/jas.2009-2121.

Choi, J.S., Kwon, K.M., Lee, Y.K., Joeng, J.U., Lee, K.O., Jin, S.K., Choi, Y.I., Lee, J.J., 2018. Application of AutoFom III equipment for prediction of primal and commercial cut weight of Korean pig carcasses. Asian-Australasian journal of animal sciences 31 (10), 1670-1676. https://doi.org/10.5713/ajas.18.0240.

Coutellier, L., Arnould, C., Boissy, A., Orgeur, P., Prunier, A., Veissier, I., Meunier-Salaün, M.-C., 2007. Pig's responses to repeated social regrouping and relocation during the growing-finishing period. Applied Animal Behaviour Science 105 (1-3), 102-114. https://doi.org/10.1016/j.applanim.2006.05.007.

D'Eath, R.B., 2005. Socialising piglets before weaning improves social hierarchy formation when pigs are mixed post-weaning. Applied Animal Behaviour Science 93 (3-4), 199211. https://doi.org/10.1016/j.applanim.2004.11.019.

Hurvich, C.M., Tsai, C.-L., 1989. Regression and Time Series Model Selection in Small Samples. Biometrika 76 (2), 297. https://doi.org/10.2307/2336663.

McGlone, J.J., Curtis, S.E., 1985. Behavior and performance of weanling pigs in pens equipped with hide areas. Journal of animal science 60 (1), 20-24. https://doi.org/10.2527/jas1985.60120x.

Meese, G.B., Ewbank, R., 1973. The establishment and nature of the dominance hierarchy in the domesticated pig. Animal Behaviour 21 (2), 326-334. https://doi.org/10.1016/S00033472(73)80074-0. 
Milligan, B.N., Fraser, D., Kramer, D.L., 2001. Birth weight variation in the domestic pig: effects on offspring survival, weight gain and suckling behaviour. Applied Animal Behaviour Science (73), 179-191.

Milligan, B.N., Fraser, D., Kramer, D.L., 2002. Within-litter birth weight variation in the domestic pig and its relation to pre-weaning survival, weight gain, and variation in weaning weights. Livestock Production Science (76), 181-191. https://doi.org/10.1016/S0301-6226(02)00012-X.

Rushen, J., 1993. The 'coping' hypothesis of stereotypic behaviour. Animal Behaviour 45 (3), 613-615. https://doi.org/10.1006/anbe.1993.1071.

Rutherford, K.M.D., Baxter, E.M., D'Eath, R.B., Turner, S.P., Arnott, G., Roehe, R., Ask, B., Sandøe, P., Moustsen, V.A., Thorup, F., Edwards, S.A., Berg, P., Lawrence, A.B., 2013. The welfare implications of large litter size in the domestic pig I: biological factors. Animal Welfare 22 (2), 199-218. https://doi.org/10.7120/09627286.22.2.199.

Rzezniczek, M., Gygax, L., Wechsler, B., Weber, R., 2015. Comparison of the behaviour of piglets raised in an artificial rearing system or reared by the sow. Applied Animal Behaviour Science 165, 57-65. https://doi.org/10.1016/j.applanim.2015.01.009.

Schmitt, O., O'Driscoll, K., Boyle, L.A., Baxter, E.M., 2019. Artificial rearing affects piglets pre-weaning behaviour, welfare and growth performance. Applied Animal Behaviour Science 210, 16-25. https://doi.org/10.1016/j.applanim.2018.10.018.

Schrøder-Petersen, D.L., Simonsen, H.B., 2001. Tail biting in pigs. Veterinary journal 162 (3), 196-210. https://doi.org/10.1053/tvjl.2001.0605.

Schwarz, G., 1978. Estimating the Dimension of a Model, $1 \mathrm{p}$.

Scollo, A., Di Martino, G., Bonfanti, L., Stefani, A.L., Schiavon, E., Marangon, S., Gottardo, F., 2013. Tail docking and the rearing of heavy pigs: the role played by gender and the presence of straw in the control of tail biting. Blood parameters, behaviour and skin lesions. Research in veterinary science 95 (2), 825-830. https://doi.org/10.1016/j.rvsc.2013.06.019.

Smith, F., Clark, J.E., Overman, B.L., Tozel, C.C., Huang, J.H., Rivier, J.E.F., Blikslager, A.T., Moeser, A.J., 2010. Early weaning stress impairs development of mucosal barrier function in the porcine intestine. American journal of physiology. Gastrointestinal and liver physiology 298 (3), G352-63. https://doi.org/10.1152/ajpgi.00081.2009.

Statham, P., Green, L., Mendl, M., 2011. A longitudinal study of the effects of providing straw at different stages of life on tail-biting and other behaviour in commercially housed pigs. Applied Animal Behaviour Science 134 (3-4), 100-108. https://doi.org/10.1016/j.applanim.2011.08.009. 
Taylor, N.R., Parker, R.M.A., Mendl, M., Edwards, S.A., Main, D.C.J., 2012. Prevalence of risk factors for tail biting on commercial farms and intervention strategies. Veterinary journal 194 (1), 77-83. https://doi.org/10.1016/j.tvjl.2012.03.004.

Tomiyama, M., Kubo, S., T., T., Suzuki, K., 2011. Evaluation of genetic trends and determination of the optimal number of cumulative records of parity required in reproductive traits in a Large White pig population. Animal science journal 82 (5), 621626. https://doi.org/10.1111/j.1740-0929.2011.00889.x.

van Beirendonck, S., Schroijen, B., Bulens, A., van Thielenab, J., Driessena, B. (Eds.), 2015. A solution for high production numbers in farrowing units?

Veit, C., Traulsen, I., Hasler, M., Tölle, K., Burfeind, O., grosse Beilage, E., Krieter, J., 2016. Influence of raw material on the occurrence of tail-biting in undocked pigs. Livestock Science 191, 125-131. https://doi.org/10.1016/j.livsci.2016.07.009.

Vos, M. de, Huygelen, V., Willemen, S., Fransen, E., Casteleyn, C., van Cruchten, S., Michiels, J., van Ginneken, C., 2014. Artificial rearing of piglets: Effects on small intestinal morphology and digestion capacity. Livestock Science 159, 165-173. https://doi.org/10.1016/j.livsci.2013.11.012.

Weary, D.M., Appleby, M.C., Fraser, D., 1999. Responses of piglets to early separation from the sow. Applied Animal Behaviour Science 63 (4), 289-300. https://doi.org/10.1016/S0168-1591(99)00021-0.

Weary, D.M., Jasper, J., Hötzel, M., 2008. Understanding weaning distress. Applied Animal Behaviour Science 110 (1-2), 24-41. https://doi.org/10.1016/j.applanim.2007.03.025.

Weatherup, R.N., Beattie, V.E., Moss, B.W., Kilpatrick, D.J., Walker, N., 1998. The effect of increasing slaughter weight on the production performance and meat quality of finishing pigs. Animal Science 67 (3), 591-600. https://doi.org/10.1017/S1357729800033038.

Welfare Quality®, 2009. Assessment protocol for pigs.

Widowski, T.M., Torrey, S., Bench, C.J., Gonyou, H.W., 2008. Development of ingestive behaviour and the relationship to belly nosing in early-weaned piglets. Applied Animal Behaviour Science 110 (1-2), 109-127. https://doi.org/10.1016/j.applanim.2007.04.010.

Widowski, T.M., Yuan, Y., Gardner, J.M., 2005. Effect of accommodating sucking and nosing on the behaviour of artificially reared piglets. Laboratory animals 39 (2), 240-250. https://doi.org/10.1258/0023677053739701.

Wolf, J., Žáková, E., Groeneveld, E., 2008. Within-litter variation of birth weight in hyperprolific Czech Large White sows and its relation to litter size traits, stillborn piglets and losses until weaning. Livestock Science 115 (2-3), 195-205. https://doi.org/10.1016/j.livsci.2007.07.009. 
Worobec, E.K., Duncan, I.J.H., Widowski, T.M., 1999. The effects of weaning at 7, 14 and 28 days on piglet behaviour. Applied Animal Behaviour Science 62 (2-3), 173-182. https://doi.org/10.1016/S0168-1591(98)00225-1.

Zonderland, J.J., 2010. Talking tails: Quantifying the development of tail biting in pigs. Wageningen, $176 \mathrm{pp}$. 


\title{
PAPER FOUR
}

\section{Activity behaviour of pigs: comparison of manual and automated video analyses}

\author{
M. Gentz' ${ }^{1}$, C. Meckbach ${ }^{1}$, S. Zeidler², V. Loges ${ }^{1}$, J. Brünger ${ }^{3}$, R. Koch ${ }^{3}$ \\ and I. Traulsen ${ }^{1}$ \\ 1Department of Animal Sciences, Livestock Systems, Georg-August-University, \\ Albrecht-Thaer-Weg 3, 37075 Göttingen, Germany \\ 2Department of Animal Sciences, Breeding Informatics, Georg-August-University, \\ Margarethe von Wrangell-Weg 7, 37075 Göttingen, Germany \\ ${ }^{3}$ Department of Computer Science, Christian-Albrechts-University, Hermann- \\ Rodewald-Str. 3, 24118 Kiel, Germany
}

Corresponding author: M. Gentz (maria.gentz@uni-goettingen.de)

Submitted to Computers and Electronics in Agriculture, 2020 


\section{Highlights}

- Behaviour observation by non-invasive methods

- Acceptable level of agreement between manual and automated video analyses

- Activity recording by distance measurement

- Rearing pigs show two activity peaks

- Activity behaviour changes during rearing

\section{Abstract}

Monitoring the activity behaviour of pigs is becoming increasingly important since changes in behaviour indicate reduced animal welfare, diseases or even an upcoming outbreak of tail biting. The behaviour of pigs in a conventional rearing system was analysed and evaluated manually and automatically at the beginning, middle and end of rearing. The manual analysis was conducted using an ethogram, which divides behaviours into inactive and active. The activities were assessed every 12 minutes by the point sampling method. Each type of behaviour was given a weighting which expressed the extend of movement of a pig, and the sum of movements resulted in an activity index (Al). The automatic evaluation was based on a computer vision algorithm which fitted ellipses as pigs. The evaluation was focussed on the centres of the fitted ellipses. The movement of the pigs was recorded (distance index (DI)) by linking the nearest neighbour ellipses (centres). Manually, an average of 12.65 pigs were identified per pen, the algorithm found 10.67 pigs and after distance measurement, 9.84 pigs were detected. The results show that automated analysis has a high accuracy and is only limited by pigs obscured by objects. Nevertheless, a comparison of the trends of the manual and automated analyses showed a match of $78.8 \%$ per hour. Moreover, AI and DI were divided into inactive, active and very active (activity) levels, for which the methods showed an agreement of $69.2 \%$ (end of rearing). It was also possible to conclude the animals' behaviour from the distance moved per pen.

Keywords: Activity; algorithm; nearest neighbour; activity index; behaviour analysis; distance index 


\section{Introduction}

Behavioural observations in modern animal husbandry are becoming increasingly important. The assessment of behavioural changes to prevent abnormal behaviour or diseases is not only important for the animals but also for the profitability of the farms (Weary et al., 2009). Live monitoring of animal behaviour using sensor technologies is becoming increasingly available (Rushen et al., 2012). Automated analysis can assess movement within groups without using the biases which occur with direct observation. New technologies lead to high accuracy and in addition is less invasive (Rushen et al., 2012). Video observations do not interfere with the animals or farm routines. Manual evaluation of video data is very complex and time-consuming. This can be simplified considerably with the help of computer vison algorithms. Low-cost cameras, in combination with image analysis technology, can be used to quickly quantify the animals' behaviour (Wathes et al., 2008). In order to evaluate the activity of pigs using analysis software it is nevertheless necessary to correctly interpret the visual scene using image analysis techniques (Oczak et al., 2014). Automated analysis enables increased objectivity and the detectability of small changes in behaviour (Oczak et al., 2014). One method of automated image analysis is the activity index of Bloemen et al. (1997). This technique allows the segmentation and quantification of the behaviour of the animals and measures the responses of the animals to their environment (Bloemen et al., 1997). This type of analysis records changes in activity at group level but does not classify individual behaviour patterns.

The behaviour of the pigs is an indicator of animal welfare and health, which is related to a good performance (McGlone, 1991). The level of animal welfare can be derived from the exploratory behaviours and the requirement of obtaining feeding (Presto et al., 2008). Farmed animals do not need to protect themselves from predators or look for feed nevertheless they perform explorative behaviour (Docking et al., 2008). The behaviour of pigs can be divided into active and inactive behaviour (Docking et al., 2008). While in inactive phases the pigs tend to rest or to sleep; there are various forms of activity. Starting with feed intake, social and sexual behaviour, playing or explorative behaviour are exercised throughout the day. Social behaviour contains body care and contacts with other pigs, which includes for example rank fights (Meyer et al., 2006; van Putten, 1978; Wechsler et al., 1991). The exploratory behaviour of the pigs occurs frequently (Wechsler et al., 1991) and is often replaced in modern housing systems, for example, by tail biting (Hörning, 1993). The activity peaks for food intake and other active behaviour are observed in the morning and afternoon. During the winter months, this activity may be shortened according to the light season in the summer months, as both the light and dark changes as well as diurnal rhythm may have an influence on the activity of the pigs (Hörning, 1993). The nocturnal rest period can last 11-13 hours, while inactive phases during the day are about three hours (Meyer et al., 2006). In grazing or outdoor enclosures, the resting 
period of pigs is less pronounced than in those kept indoors, as these pigs naturally spend more time foraging (van Putten, 1978). The inactive phases of the pigs are initiated some time before by reduced activity, scratching and dozing while standing. Complete relaxation is finally achieved in the lateral position, in which the pig finds its sleep. Occasionally, it can be observed that pigs perform walking movements with their limbs while lying down, which indicates that pigs dream while asleep (van Putten, 1978).

Previous studies have dealt with the automated evaluation of pig behaviour. The authors recorded activity with the help of an activity index. This index was based on the change in pixels and thus recorded any movement of the pigs (Costa et al., 2009; McGlone, 1991; Oczak et al., 2014) or sows (Küster et al., 2020). Thus, their output was quantified as the number of pixels moved each second of the recorded videos in relation to the total number of pixels in the image (Costa et al., 2009; McGlone, 1991; Oczak et al., 2014).

The automatic recording of activity changes or the filtering of unusually high or low activity can provide information on the well-being of the animals (Presto et al., 2008). Behavioural analysis can also be used as a predictor of abnormal behaviour such as tail biting (Larsen et al., 2016; Statham et al., 2009). This is of particular interest for the future housing of undocked pigs (Gentz et al., 2020) as tail biting is still a large problem with several risk factors (Valros, 2018).

The aim of the study was to evaluate a method of automated activity behaviour analysis of rearing pigs. For this analysis, a comparison of manual and automatically video analysis was conducted.

\section{Material and Methods}

\subsection{Data collection}

The data was acquired at the research farm in Futterkamp of the Chamber of Agriculture of Schleswig-Holstein located in northern Germany. The dataset comprises 260 rearing pigs. For observation AXIS M30-VE Network cameras were used, one camera recorded two pens. The pigs were observed during the rearing period when they were aged between four and ten weeks. 13 pigs were housed together with a space allowance of $0.44 \mathrm{~m}^{2}$ per pig. They were fed ad libitum with a commercial feed ration. Each pen had three feeding troughs with four places and two bowl drinkers (Figure 1). The pigs had an additional trough with straw or peas to enrich the housing environment and they received wood and jute bags or ropes to explore. The temperature was kept constant (about 28 degrees at the beginning of the rearing period) with the help of a gas(-powered) heater. 


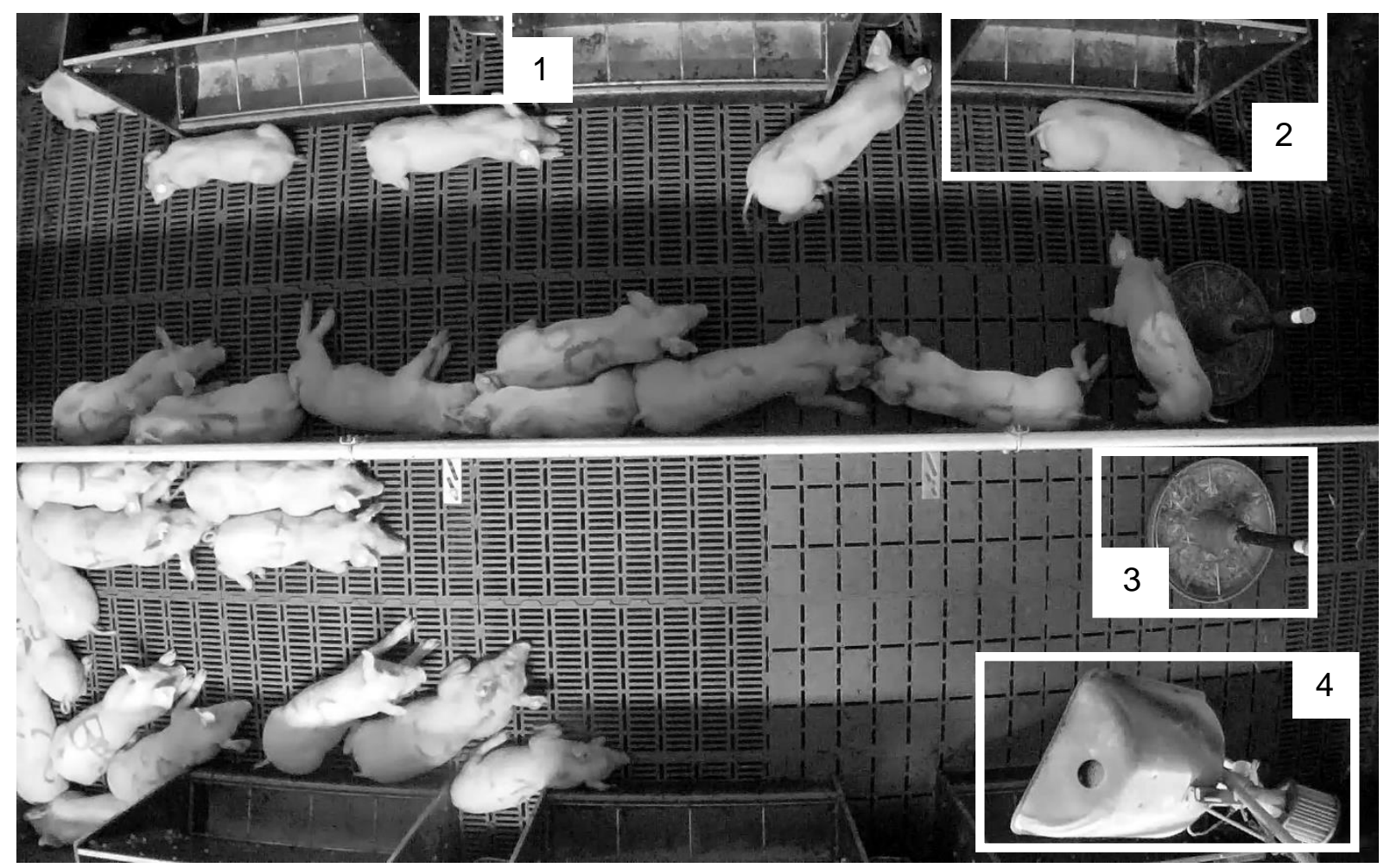

Figure 1. Pen design: two pens (each $1.27 \times 4.48=5.69 \mathrm{~m}^{2}$ ) recorded by one camera with an identical pen design; $1=$ bowl drinker, $2=$ feeding trough, $3=$ additional feeding trough, $4=$ gas heater.

\subsection{Manual video analysis}

\subsubsection{Data analysis}

For the data analysis, one day at the beginning, in the middle and at the end of the rearing period were used (day 3, 17 and 31). On all three days, the recordings between 06:00 and 18:00 hrs were used. The manual video analysis was conducted with the Noldus Observer XT 14 software. An ethogram was developed to evaluate the behaviour (Table 1). The behaviours were divided into active and inactive whereas inactive pigs were lying, and active pigs were sitting, eating, drinking, walking/standing or interacting with enrichment material or other pigs. The lying included the lateral and abdominal position, but also the cluster position, in which a certain lying position is not necessarily precisely defined. Although sitting is a compromise behaviour, it was regarded as active (Wennrich, 1978). Sitting serves as a transition between standing and lying (Lang, 2004; Schultze, 2008) and may also indicate reduced well-being (Arellano et al., 1992). All behaviour patterns except for lying were defined as active. In addition to the ethogram, deviations were recorded, including pigs concealed by the heater or feed pipes, but also when the pigs were outside the edge of the picture. 
Table 1. Ethogram used for manual video analysis (following Hübner-Weitz (2010), Klein (2016) and Pflanz (2007)).

\begin{tabular}{ll}
\hline Active behaviour & Definition \\
Sitting & claws of the extended front limbs touch the ground, \\
ischial tuberosity and hind limbs also rest on the \\
ground, the head is kept off the ground \\
mouth is at the feeding trough \\
mouth is at the drinking trough \\
Drinking \\
only the claws touch the ground, at least three \\
limbs are loaded and are stretched out, the head is \\
kept off the ground, or it is on the ground with the \\
trunk \\
exploring, plying, sniffing, pounding, biting with the \\
mouth/trunk at enrichment material \\
biting, riding, sniffing, fighting behaviour, belly- \\
nosing \\
Interactions with enrichment material
\end{tabular}

The data acquisition was carried out using the point sampling method (Martin and Bateson, 2006) during daytime. Every 12 minutes a picture of the pen was taken, and the respective number of animals per behaviour was recorded.

\subsubsection{Activity Index}

To enable comparability with the automatic analysis method, an activity index was developed.

For each type of behaviour, a ranked weighting was established, which expressed the extent to which this activity represented movements of the animals (Table 2). 
Table 2. Activity weighting of different behavioural patterns.

\begin{tabular}{ll}
\hline Behaviour & Activity weighting \\
\hline Lying & 0.01 \\
Sitting & 0.03 \\
Eating/Drinking & 0.1 \\
Walking/Standing & 0.65 \\
Interaction with enrichment material & 0.65 \\
Interaction with pen members & 1 \\
\hline
\end{tabular}

The sum of the activity weightings $\left(\mathrm{w}_{\mathrm{i}}\right)$ for each pig (i) results in an activity index per pen (Al), which can be a maximum of 13 (13 pigs per pen).

$$
A I=\sum_{i=1}^{13} w_{i}
$$

The Al was used for further analysis.

\subsection{Automated video analysis}

\subsubsection{Automated detection of pigs}

For the automatic video analysis, the instance segmentation method described in Brünger et al. (2020) was used to detect the pigs in the individual frames of the videos. Based on manually annotated images of similar housing systems from previous studies, a deep neural network was trained to detect the positions and orientations of the pigs. The detections were parametrised as ellipses and stored in a database.

Ellipses are often used to approximate the outlines of pigs (Kashiha et al., 2013; McFarlane and Schofield, 1995; Nasirahmadi et al., 2015), since they only need five parameters and still sufficiently trace the outline of the animals without including too much background, as it is often the case with bounding boxes.

The network used is a U-Net (Ronneberger et al., 2015) architecture equipped with two heads to produce pixel embedding and a categorical, pig body, part segmentation of the input images (Figure 2). 


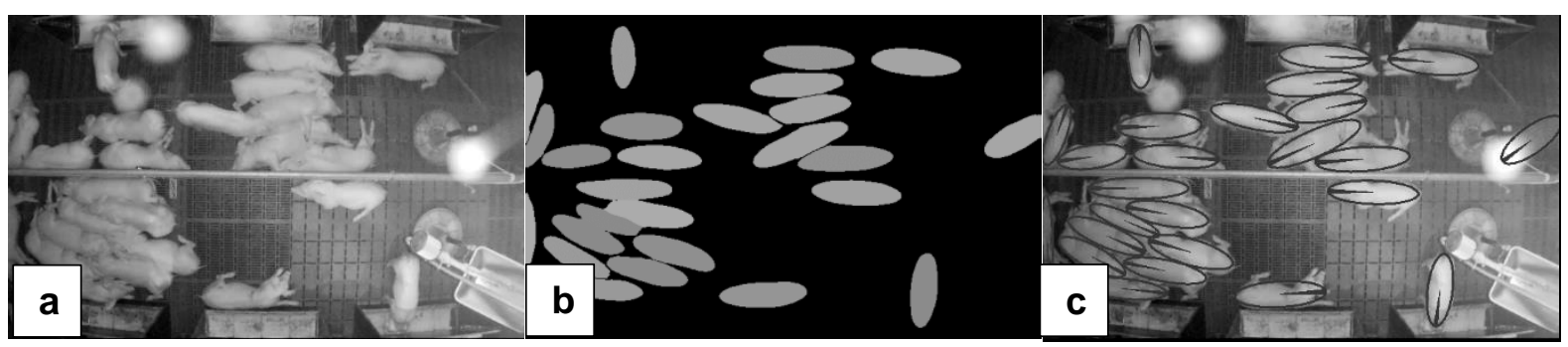

Figure 2. Example results from the neural network. From left to right: a) original input image, b) instance segmentation based on the clustering and c) the extracted ellipses. The filled part of the ellipses shows the identified orientation.

The network is trained to layout the individual pixels in the embedding space such that pixels which belong to the same animal form a cluster, and clusters representing different animals are pushed apart from each other. In a post-processing step, these clusters are extracted using the density-based clustering method HDBSCAN (Campello et al., 2013).

The simultaneously created segmentation of the input image can be used to limit the clustering to those pixels that belong to the animals and thus exclude the background. Back projecting the clusters into the image-space yields the pixel-precise segmentation of the individual animals to which the ellipses are fitted in a final step (Fitzgibbon and Fisher, 1995).

\subsubsection{Processing of automatic data}

For each time point of the manual analysis, a video length of five seconds was considered, represented as 50 frames in a sequence. For each of these frames, the predicted pigs were represented as ellipses from the previous step. In order to examine the motion of the pigs within the 5s under study, a mapping of ellipses from one frame to the following frame was established. The mapping procedure proceeded as follows for all consecutive frames. To map ellipses of frame $t$ to those of the following frame $t+1$, the Euclidian distances $d\left(e_{i t}, e_{j t+1}\right)$ of all ellipses $e_{i t}(i=1,2 \ldots)$ of frame $t$ and all ellipses $e_{j+1}(j=1,2 \ldots)$ of frame $t+1$ based on the ellipses centres were calculated. Afterwards, the ellipse pairs were sorted in ascending order according to their distance and performed a "greedy" (locally optimal choice) selection of ellipse pairs. Starting from the minimal distance $d_{\text {min }}$, the corresponding pair of ellipses was selected and the ellipses put in a list $L$. The next ellipse pair was then assessed and the pair put in $L$ if none of the single ellipses of the pair was already contained in $\mathrm{L}$. The procedure continued until the distance of a pair exceeded 150 pixels. Setting a maximal distance threshold of 150 pixel was mandatory since this was the maximal, biologically reliable distance a pig can move in the time from one frame to another. For the tracing back of the ellipses throughout all sequential frames, the ID of the precursor ellipse as well as the ID of the first ancestor ellipse of each mapping was stored. Finally, for each time point, a list of mapped ellipses resulted, the number of frames the mapping assessed and the distances the ellipses moved along the mapping. 
The automatically detected ellipses were mapped and then filtered. Only pigs that were mapped at least 48 times in succession were considered.

\subsubsection{Distance Index}

Each determined ellipse was mapped, and the distance covered was then recorded as described above. The sum of the distances $\left(d_{i}\right)$ over all pigs (i) in the pen per sample point was combined to a distance index.

$$
D I=\sum_{i=1}^{13} d_{i}
$$

The DI was used for further analysis.

\subsection{Comparison of manual (AI) and automated analysis (DI)}

The statistical analysis was carried out using SAS 9.4 (SAS R Institute Inc, 2008). The data from the manual and automated evaluation were compared on a pen basis (Al vs. DI), as there was no individual animal tracking with a unique identification. In total, 3,058 sample points and 152,900 frames were used.

\subsubsection{Pig detections per image}

The first step was to compare how many pigs had been detected manually and automatically by the algorithm and to determine the number of pigs still found after mapping. A boxplot was used for this analysis.

\subsubsection{Course of activity over rearing period}

To investigate the course of the mean activity per pen and day during the rearing period (beginning, middle, end), the mean values per sample point were calculated by the MEANS procedure for all pens. Furthermore, the mean values per hour were calculated.

\subsubsection{Gradient}

The values representing the activity level were manually expressed by Al and automatically expressed by DI. The difference quotient (DQ) was used to record whether the activity level had decreased or increased from hour to hour during the day regarding the manual, respectively the automated, analysis.

$$
D Q=\frac{D I_{t+1}-D I_{t}}{A I_{t+1}-A I_{t}}
$$


To compare the gradients (Grad) of $\mathrm{Al}$ and $\mathrm{DI}$, the values of $\mathrm{DQ}$ were distinguished into increasing $(+1)$, constant $(0)$ or decreasing $(-1)$. The FREQ procedure was used to calculate the identical trends of $\mathrm{Al}$ and $\mathrm{DI}$ for the sample points (every 12 minutes) as well as the trends per hour.

$$
\operatorname{Grad}=\left\{\begin{array}{c}
1, \text { if } D Q>0 \\
0, \text { if } D Q=0 \\
-1, \text { otherwise }
\end{array}\right.
$$

The mean activity per sample point respectively per hour over all pens was calculated.

\subsubsection{Distribution of activity (AI) and distance index (DI)}

The activity levels of the pens were analysed by UNIVARIATE procedure, to evaluate inactive, active and very active behaviour. The distributions of Al and DI were structured and compared. The 10th percentile limited the border to inactive behaviour and the 90th percentile was the limit for very active behaviour. Thereby, the distribution was calculated for beginning, middle and end of the rearing period.

\section{Results}

\subsection{Pig detections per image}

The comparison of the detections shows that most pigs per pen were found manually (mean 12.65, Figure 3). The automatic evaluation found on average 10.67 pigs, whereas 9.84 pigs were found after mapping. The deviations in the manual evaluation were only downwards while the whiskers automatically reached in both directions.

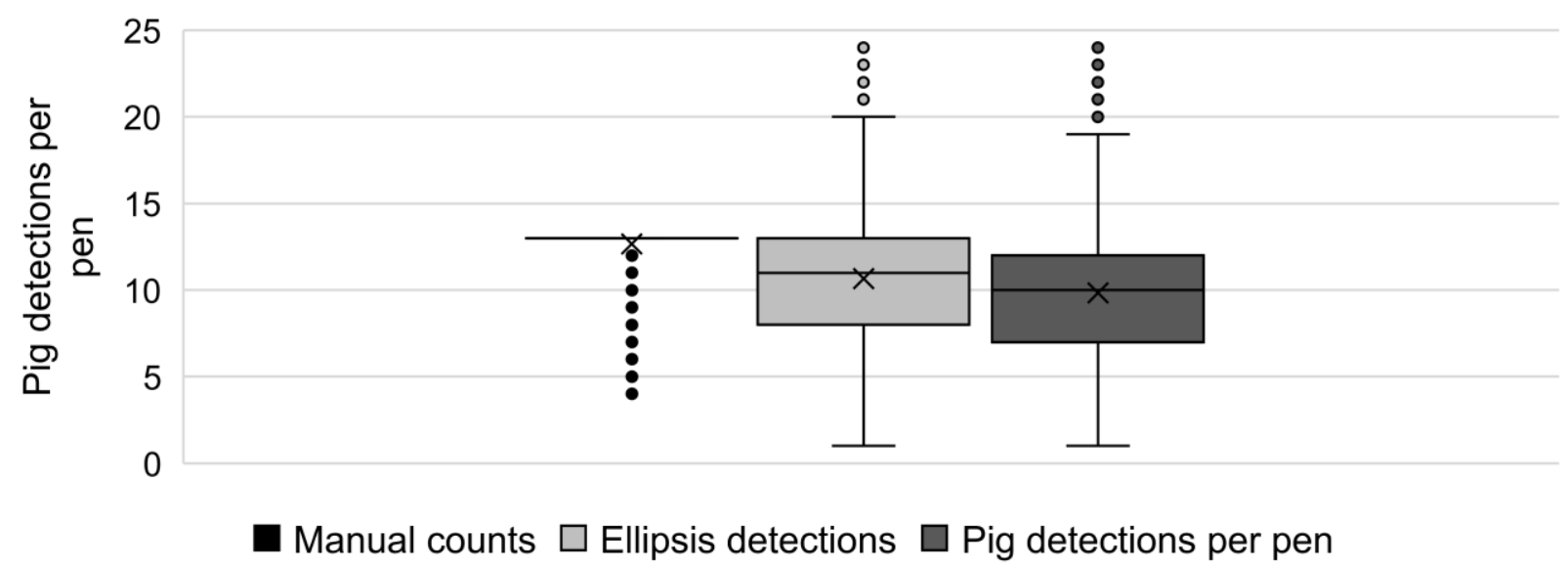

Figure 3. Pig detections per pen: boxplot analysis of manual counts, ellipsis detections and ellipsis detections after mapping. 


\subsection{Course of activity over rearing period}

At the beginning of the rearing period, the Al showed a two-edged curve per day (Figures $4 a$ and $4 \mathrm{~b}$ ). The first peak can be seen around midday and the second peak can be seen in the afternoon. The DI and Al course were comparable, but the peaks of the DI were less striking.

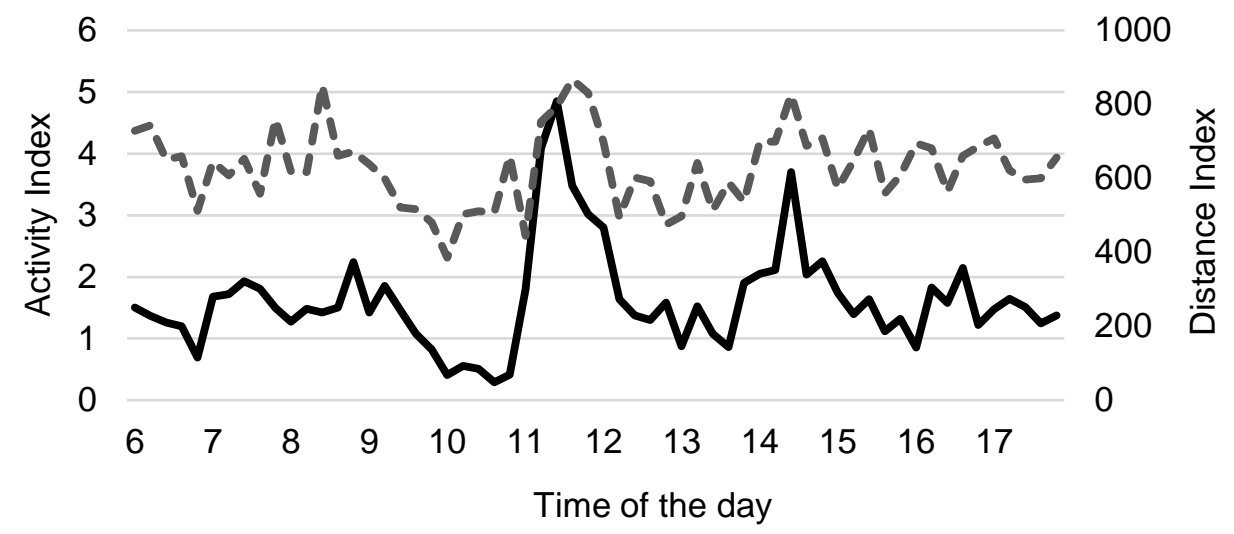

- Activity Index - - D Distance Index

Figure 4a. Course of activity over the day, mean per sample point: beginning of rearing period.

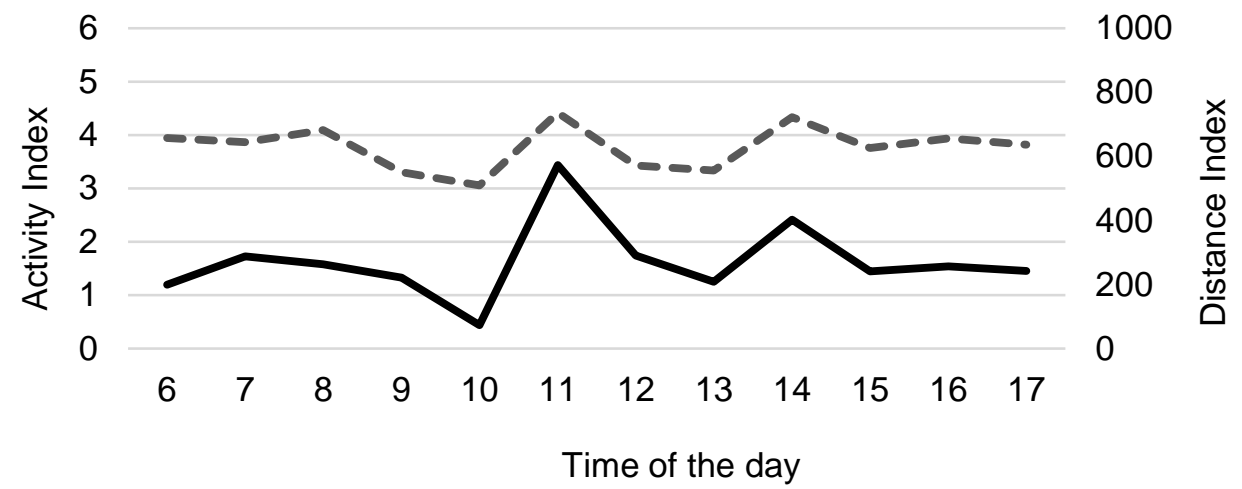

- Activity Index - - - Distance Index

Figure $\mathbf{4 b}$. Course of activity over the day, means per hour: beginning of rearing period.

In the middle of rearing, the curve of the Al per day showed two peaks: in the morning and in the evening (Figures $5 \mathrm{a}$ and $5 \mathrm{~b}$ ). The peaks of the DI can be found in the afternoon and, in the evening, as well. 


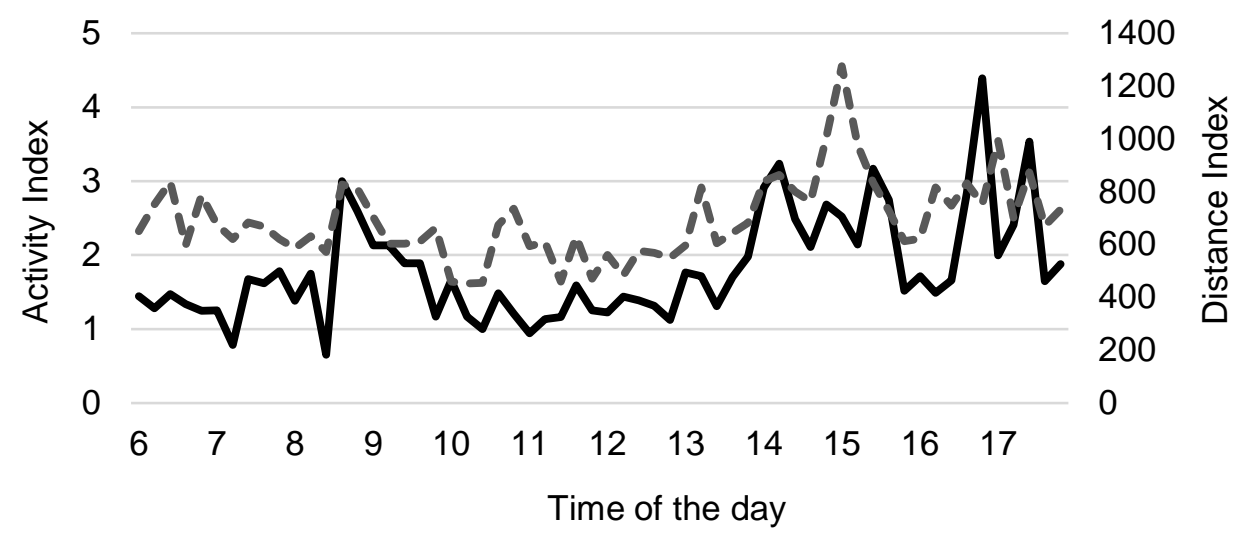

Activity Index _- Distance Index

Figure 5a. Course of activity over the day, mean per sample point: middle of rearing period.

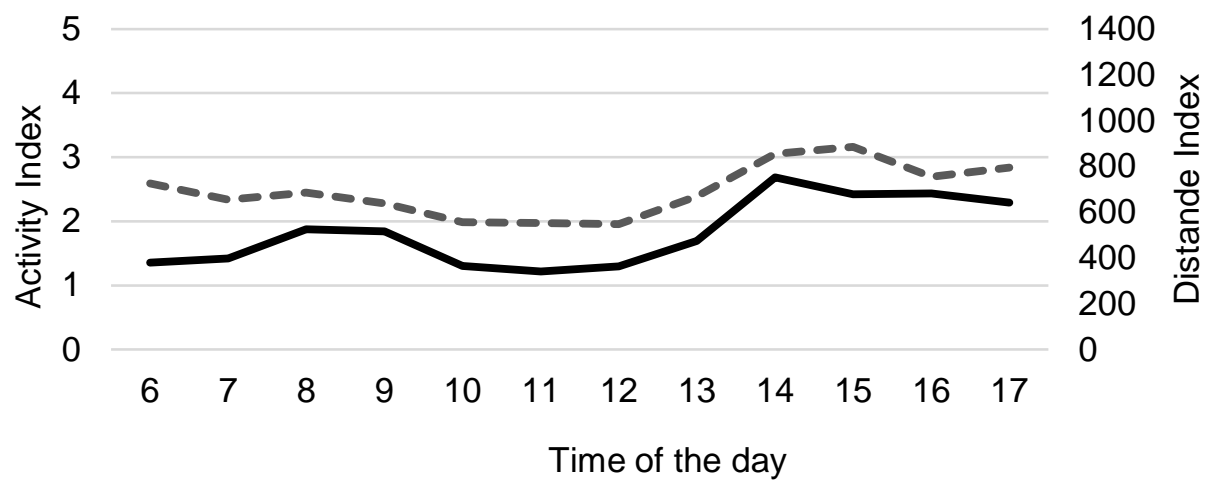

- Activity Index _- Distance Index

Figure $5 \mathbf{b}$. Course of activity over the day, means per hour: middle of rearing period.

At the end of rearing period, there was an activity peak in the later morning and in the afternoon, which was shown by Al and DI (Figures 6a and 6b)

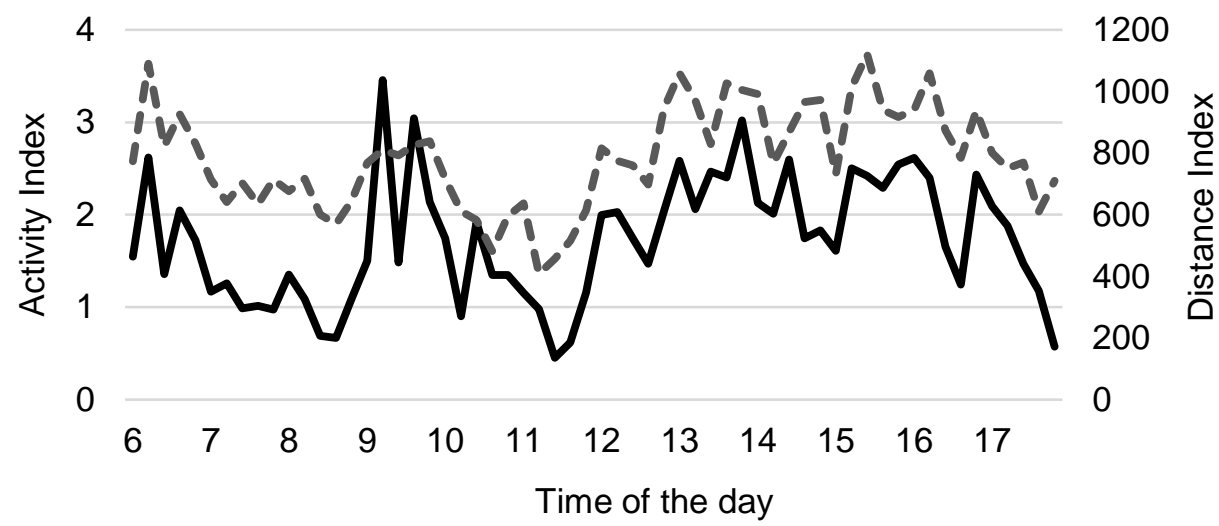

-Activity Index $\quad-\infty$ Distance Index

Figure 6a. Course of activity over the day, mean per sample point: end of rearing period. 


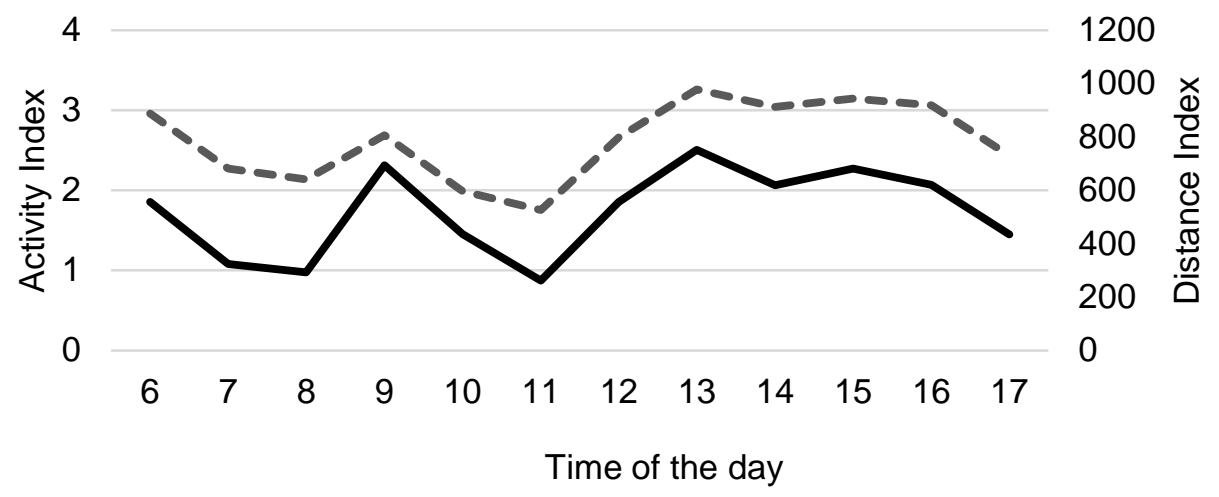

- Activity Index _- - Distance Index

Figure $6 \mathrm{~b}$. Course of activity over the day, means per hour: end of rearing period.

In general, Figures $4 b, 5 b$ and $6 b$, using averages per hour, smooth the course of the activity per day. Using sample points, Figures $4 a, 5 a$ and $6 a$ show more bias. Comparing the courses of activity of the beginning, middle and end of the rearing period, the Al showed a decreasing tendency of activity during the rearing period. The DI, in contrast, displayed the lowest values at the beginning of rearing followed by the end and the highest activity was assessed in the middle. Moreover, activity during the afternoon in the middle and end of rearing period is at a higher level than at the beginning. The gradient per hour (Figures $4 b, 5 b, 6 b$ ) was used for the comparison of the general activity levels (3.3).

\subsection{Gradient}

The gradient was calculated for the comparison of the trends between Al and DI at the respective time during rearing (Table 4). Comparing the values every 12 minutes, the agreement reached values of maximum $50.4 \%$ (middle). After calculating the mean values per hour and comparing their trends, a high degree of agreement can be observed (up to $100 \%$ at the end of rearing). These findings confirm the virtual interpretation presented in Figures $4 b$, $5 b$ and $6 b$.

Table 4. Comparison of the proportion of identical gradients per time point (beginning, middle, end of rearing period).

\section{Relative frequency of identical trend}

per sample point

(Figures 4a, 5a, 6a) means per hour

(Figures 4b, 5b, 6b)

\begin{tabular}{llr}
\hline Beginning of rearing period & $44.4 \%$ & $81.8 \%$ \\
Middle of rearing period & $50.4 \%$ & $54.5 \%$ \\
End of rearing period & $52.0 \%$ & $100 \%$ \\
\hline$\varnothing$ & $\mathbf{4 8 . 9} \%$ & $\mathbf{7 8 . 8 \%}$
\end{tabular}




\subsection{Distribution of activity (Al) and distance index (DI)}

The analysis of the distribution showed that the inactive percentile (10\%) only contained lying pigs, without presupposing this (see Table $5, \mathrm{Al}=0.13$ ), regarding all three time points of rearing. The 90th percentile (very active level $(\mathrm{Al})$ ) increased from the beginning to the middle and decreased again. The values of the DI increased slightly during rearing time at both activity levels (inactive and very active). The matching of the distributions within the respective activity level went up to $69.2 \%$ (end).

Table 5. Distribution of Activity Index (Al) and Distance Index (DI) over the three time points (beginning, middle, end of rearing period) and comparison of the matching of $\mathrm{Al}$ and $\mathrm{DI}$ per pen and time point.

\begin{tabular}{|c|c|c|c|c|c|}
\hline & & & Activity level & & \\
\hline & & $\begin{array}{l}\text { Inactive } \\
\leq 10 \text { th } \\
\text { percentile }\end{array}$ & $\begin{array}{c}\text { Active } \\
\text { 90th percentile } \\
>x> \\
\text { 10th percentile }\end{array}$ & $\begin{array}{c}\text { Very Active } \\
\geq \text { 90th } \\
\text { percentile }\end{array}$ & $\begin{array}{l}\text { Matching of } \\
\text { Al and DI } \\
\text { per pen and } \\
\text { time point }\end{array}$ \\
\hline \multirow{2}{*}{$\begin{array}{l}\text { Beginning of } \\
\text { rearing period }\end{array}$} & Al & 0.13 & $0.13>x>4.06$ & 4.06 & \multirow{2}{*}{$58.2 \%$} \\
\hline & DI & 177.66 & $177.66>x>1110.72$ & 1110.72 & \\
\hline \multirow{2}{*}{$\begin{array}{l}\text { Middle of } \\
\text { rearing period }\end{array}$} & Al & 0.13 & $0.13>x>4.3$ & 4.3 & \multirow{2}{*}{$65.6 \%$} \\
\hline & DI & 223.83 & $223.83>x>1247.28$ & 1247.28 & \\
\hline \multirow{2}{*}{$\begin{array}{l}\text { End of } \\
\text { rearing period }\end{array}$} & Al & 0.13 & $0.13>x>3.86$ & 3.86 & \multirow{2}{*}{$69.2 \%$} \\
\hline & DI & 312.80 & $312.80>x>1338.01$ & 1338.01 & \\
\hline
\end{tabular}

\section{Discussion}

\subsection{Pig detections per image}

The manually and automatically detected numbers of animals differ (see Figure 1). The main reason was the occasional presence of gas heaters, which covered the view of the cameras. Humans were able to count the visible animals that were visible and deduce their behaviour from the heads or the legs although the gas burner inhibited the view on the whole pig. The algorithm did not recognise an animal in these cases and therefore the average number of animals was lower. This occurred frequently especially at the beginning of the rearing period, as the heaters were removed when the animals became older. The value of the pig detections after mapping was lower because of the data processing in which mappings of less than 48 frames were deleted. 
The optimisation of the automated detections could be achieved through a possible improvement of the image material. However, the experiment was carried out under practical conditions and it was therefore not possible to completely avoid disturbances in the picture.

\subsection{Course of activity over rearing period}

The general activity level at the beginning, in the middle and at the end of rearing was different. The activity levels differed in the course of the day as shown by Costa et al. (2009) and Stukenborg et al. (2011). A normal daily routine describes the feed intake in the morning, followed by a resting period around midday, and in the afternoon the animals show social interactions until they finally come to rest (Stolba and Wood-Gush, 1989). There were two activity peaks on all three days of the rearing period in the midday hours, in the early afternoon or in the evening. Stukenborg et al. (2011) observed a two-peak curve as well while analysing agonistic behaviour in rearing pigs. A high activity level in the afternoon was also detected by Costa et al. (2009) and Caley (1997). Particularly in the older pigs at the end of the rearing, active behaviour can be observed throughout the afternoon, which is mainly related to the animals' behaviour at the feeding trough according to Fraser et al. (1991). Similarly, in the current study, almost half of the active behaviour in the late afternoon could be attributed to eating.

By comparing $\mathrm{Al}$ and $\mathrm{DI}$, especially in the morning, higher $\mathrm{DI}$ than $\mathrm{Al}$ values can often be observed proportionally. A morning active phase is typical for pigs (Caley, 1997). Because the Al contained only one frame, the behaviour could be recorded, but the level of movement was always the same for one activity. Indicating that an animal was e.g. eating, the level of activity remained the same. When the animals get up in the morning and all of them go to feed, there is usually a higher level of activity in the pen than if a single animal goes to feed in the afternoon. This aspect could not be considered by the Al. With the DI method, this was different. However, since the DI method includes several consecutive frames, the activities can be recorded at different intensities.

The evaluation of the activity on an hourly basis commonly used in livestock behaviour analysis can also be found in the literature (Costa et al., 2009; Stukenborg et al., 2011). Using averages per hour smooths the course of the activity per day. The bias which can be found in the analysis per sample point is straightened.

\subsection{Gradient}

The above-mentioned aspect of the different evaluations of individual activity levels concerning $\mathrm{Al}$ and DI was also evident when comparing trends. In this case, the most relevant activity was the behaviour of walking/standing, which was mainly responsible for differences in the value 
ranges. It was not possible to differentiate whether a pig was walking or standing on one frame (Al) whereas the DI recorded the distance (a walking pig caused higher DI than a standing pig). In addition, there were several types of lying. The pigs can be lying while sleeping or while resting (Docking et al., 2008). A sleeping pig is does not move for a length time while a resting pig does not move for a shorter time. The Al recorded inactive, whereas the DI analysed low activity. With $\mathrm{Al}$ and $\mathrm{DI}$, it was not possible to detect the way of lying, but there are differentiated algorithms to monitor pigs lying on their bellies or on their sides (Nasirahmadi et al., 2019). However, this study does not conclude information on behavioural abnormalities. Other methods for the automatic recognition of activity neither differentiate for example resting or sleeping, but lying indicates an inactive pig (Costa et al., 2009; Oczak et al., 2014). Due to the pixel change, an animal is generally defined as active when a level is exceeded, not considering whether or how many of the animals were resting or sleeping (Docking et al., 2008). By comparing the activity on an hourly basis, activities of varying intensity can be smoothed, and comparability of rearing phases and other studies also increase.

\subsection{Distribution of activity (Al) and distance index (DI)}

The comparison of inactive and active phases of the day is normally used to evaluate activity behaviour in literature (Costa et al., 2009; Docking et al., 2008; Oczak et al., 2014). Consequently, the $\mathrm{Al}$ and $\mathrm{DI}$ were divided into inactive, active and very active phases. The development of Al during the rearing period showed a constant number of inactive pigs. An Al of 0.13 indicates that all the pigs were lying in the pen. Regarding the very active (90th) percentile of activity the Al showed the highest values in the middle of rearing, whereas the DI increased slightly over time. In the first week after weaning, the pigs explored their new environment and needed to find a new hierarchy (Friend et al., 1983). The increasing DI can result from the growth of the animals. The lower $\mathrm{Al}$ at the end of rearing might be a result of growth as well. Generally, the space allowance in the pens decreases over time so there might be less space for a high activity level. However, both indices showed a matching of up to $70 \%$.

\subsection{Further studies}

Automatically video analysis has great potential to measure activity within groups of pigs. A possible tracking algorithm of individuals would improve this method (Rushen et al., 2012). The non-invasive methods can help to optimise animal welfare by analysing the animals with a very high accuracy (Rushen et al., 2012). As the activity of pigs changes before a tail biting outbreak (Larsen et al., 2016; Statham et al., 2009), quick video analysis systems could help to prevent abnormal behaviour or diseases. A combination of body part detection (e.g. the tail) (Psota et al., 2019) and scoring of tail lesions (Brünger et al., 2019) might offer potential for an early warning system for tail biting. 


\section{Conclusion}

Comparing the manual (AI) and automatic (DI) evaluation displayed similar activity patterns at the three points of time during the rearing period. The trends that represent the daily course achieve a high degree of agreement (up to $78.8 \%$ ) on an hourly basis, which is commonly used in livestock behaviour analysis. The automatic investigation of separate activity levels confirmed the manual method, especially in the inactive phases. The range of values that the DI adopts for a certain behaviour could be investigated in further studies to be able to obtain more precise information on animal behaviour. Moreover, adequate definitions of manual and automatic behaviours are necessary to develop a practical, implementable tool (e.g. to detect tail biting).

Author Contributions: Maria Gentz: Conceptualisation, Methodology, Software, Validation, Formal analysis, Investigation, Data curation, Writing - original draft, Writing - review and editing, Visualization. Cornelia Meckbach: Methodology, Software, Validation, writing original draft, Writing - review and editing. Sebastian Zeidler: Methodology, Software, Validation, Writing - review and editing. Vivien Loges: Methodology, Software, Validation. Johannes Brünger: Methodology, Software, Writing - original draft. Reinhard Koch: Project administration, Supervision. Imke Traulsen: Conceptualisation, Methodology, Validation, Resources, Writing - review and editing, Supervision, Project administration, Funding acquisition. All authors have read and agreed to the published version of the manuscript.

Acknowledgements: This research was funded by the Federal Office for Agriculture and Food of Germany and the Landwirtschaftliche Rentenbank (project no.: 2817205413; 758914) and by the $\mathrm{H}$. Wilhelm Schaumann Foundation.

Ethics statement: All the pigs were housed in accordance with EU (European Directive 2008/120/EC) and national law (Pig Welfare Act (18/05/2016), Pig Welfare and Pig Husbandry Ordinance (05/02/2014).

Declaration of interest: The authors declare no conflict of interest. The funders did not play a role in the design of the study, in the collection, analyses, or interpretation of data, in the writing of the manuscript, or in the decision to publish the results. 


\section{References}

Arellano, P.E., Pijoan, C., Jacobson, L.D., Algers, B., 1992. Stereotyped behaviour, social interactions and suckling pattern of pigs housed in groups or in single crates. Applied Animal Behaviour Science 35 (2), 157-166. https://doi.org/10.1016/0168-1591(92)90006W.

Bloemen, H., Aerts, J.M., Berckmans, D., Goedseels, V., 1997. Image analysis to measure activity index of animals. Equine veterinary journal (23), 16-19. https://doi.org/10.1111/j.2042-3306.1997.tb05044.x.

Brünger, J., Dippel, S., Koch, R., Veit, C., 2019. 'Tailception': using neural networks for assessing tail lesions on pictures of pig carcasses. Animal 13 (5), 1030-1036. https://doi.org/10.1017/S1751731118003038.

Brünger, J., Gentz, M., Traulsen, I., Koch, R., 2020. Panoptic Instance Segmentation on Pigs, 18 pp. http://arxiv.org/pdf/2005.10499v1.

Caley, P., 1997. Movements, Activity Patterns and Habitat Use of Feral Pigs (Sus scrofa) in a Tropical Habitat. Wildlife Research 24 (1), 77. https://doi.org/10.1071/WR94075.

Campello, R.J.G.B., Moulavi, D., Sander, J., 2013. Density-Based Clustering Based on Hierarchical Density Estimates, in: Hutchison, D., Kanade, T., Kittler, J. (Eds.), Advances in Knowledge Discovery and Data Mining: 17th Pacific-Asia Conference, PAKDD 2013, Gold Coast, Australia, April 14-17, 2013, Proceedings, Part II, vol. 7819. Springer Berlin Heidelberg, Berlin/Heidelberg, pp. 160-172.

Costa, A., Borgonovo, F., Leroy, T., Berckmans, D., Guarino, M., 2009. Dust concentration variation in relation to animal activity in a pig barn. Biosystems Engineering 104 (1), 118124. https://doi.org/10.1016/j.biosystemseng.2009.05.009.

Docking, C.M., van de Weerd, H.A., Day, J.E.L., Edwards, S.A., 2008. The influence of age on the use of potential enrichment objects and synchronisation of behaviour of pigs. Applied Animal Behaviour Science 110 (3-4), 244-257. https://doi.org/10.1016/j.applanim.2007.05.004.

Fitzgibbon, A.W., Fisher, R.B., 1995. A Buyer's Guide to Conic Fitting.: The technique used for ellipse fitting is the first one described in this summary paper. Proc.5th British Machine Vision Conference, 1995, Birmingham.

Fraser, D., Phillips, P.A., Thompson, B.K., Tennessen, T., 1991. Effect of straw on the behaviour of growing pigs. Applied Animal Behaviour Science 30 (3-4), 307-318. https://doi.org/10.1016/0168-1591(91)90135-K.

Friend, T.H., Knabe, D.A., Tanksley, T.D., 1983. Behavior and performance of pigs grouped by three different methods at weaning. Journal of animal science 57 (6), 1406-1411. https://doi.org/10.2527/jas1983.5761406x. 
Gentz, M., Lange, A., Zeidler, S., Lambertz, C., Gauly, M., Burfeind, O., Traulsen, I., 2020. Tail Lesions and Losses of Docked and Undocked Pigs in Different Farrowing and Rearing Systems. Agriculture 10 (4), 130. https://doi.org/10.3390/agriculture10040130. Hörning, B., 1993. Artgemässe Schweinehaltung: Grundlagen und Beispiele aus der Praxis, 2nd ed. Müller, Karlsruhe, 253 pp.

Hübner-Weitz, K., 2010. Auswirkungen klinischer und subklinischer Krankheit auf ethologische und klinisch-chemische Merkmale beim Schwein. Dissertation. Gießen.

Kashiha, M., Bahr, C., Haredasht, S.A., Ott, S., Moons, C.P.H., Niewold, T.A., Ödberg, F.O., Berckmans, D., 2013. The automatic monitoring of pigs water use by cameras. Computers and Electronics in Agriculture 90, 164-169. https://doi.org/10.1016/j.compag.2012.09.015.

Klein, S., 2016. Auswirkungen und Folgen einer frühen Sozialisierung von Ferkeln auf das Verhalten vom Ferkel bis zum Mastschwein sowie auf das Schwanzbeißen. Dissertation. München.

Küster, S., Kardel, M., Ammer, S., Brünger, J., Koch, R., Traulsen, I., 2020. Usage of computer vision analysis for automatic detection of activity changes in sows during final gestation. Computers and Electronics in Agriculture 169, 105177. https://doi.org/10.1016/j.compag.2019.105177.

Lang, E., 2004. Einfluss einer Echinacea-Fütterung auf Immunstatus und Verhalten bei Ferkeln in den ersten Lebenswochen. Dissertation. München.

Larsen, M.L.V., Andersen, H.M.-L., Pedersen, L.J., 2016. Can tail damage outbreaks in the pig be predicted by behavioural change? Veterinary journal 209, 50-56. https://doi.org/10.1016/j.tvjl.2015.12.001.

Martin, P., Bateson, P.P.G., 2006. Measuring behaviour: An introductory guide, 2nd ed. Cambridge Univ. Press, Cambridge, 222 pp.

McFarlane, N.J.B., Schofield, C.P., 1995. Segmentation and tracking of piglets in images. Machine Vision and Applications 8 (3), 187-193. https://doi.org/10.1007/BF01215814.

McGlone, J.J., 1991. Techniques for evaluation and quantification of pig reproductive, ingestive, and social behaviors. Journal of animal science 69 (10), 4146-4154. https://doi.org/10.2527/1991.69104146x.

Meyer, E., Jahn, I., Seeber, B., Scheinert, R., Löwig, M., 2006. Schriftenreihe Haltungsalternativen Schwein. Schriftenreihe der Sächsischen Landesanstalt für Landwirtschaft (8/2006).

Nasirahmadi, A., Richter, U., Hensel, O., Edwards, S., Sturm, B., 2015. Using machine vision for investigation of changes in pig group lying patterns. Computers and Electronics in Agriculture 119, 184-190. https://doi.org/10.1016/j.compag.2015.10.023. 
Nasirahmadi, A., Sturm, B., Edwards, S., Jeppsson, K.-H., Olsson, A.-C., Müller, S., Hensel, O., 2019. Deep Learning and Machine Vision Approaches for Posture Detection of Individual Pigs. Sensors (Basel, Switzerland) 19 (17). https://doi.org/10.3390/s19173738.

Oczak, M., Viazzi, S., Ismayilova, G., Sonoda, L.T., Roulston, N., Fels, M., Bahr, C., Hartung, J., Guarino, M., Berckmans, D., Vranken, E., 2014. Classification of aggressive behaviour in pigs by activity index and multilayer feed forward neural network. Biosystems Engineering 119, 89-97. https://doi.org/10.1016/j.biosystemseng.2014.01.005.

Pflanz, W., 2007. Tier- und umweltgerechte Haltungsverfahren in der Schweinehaltung: Gesamtheitliche Bewertung innovativer Schweinemastverfahren für Baden-Württemberg. Universität Hohenheim, Stuttgart, 249 pp.

Presto, M.H., Andersson, H.K., Folestam, S., Lindberg, J.E., 2008. Activity behaviour and social interactions of pigs raised outdoors and indoors. Archiv für Tierzucht 51 (4), 338350.

Psota, E.T., Mittek, M., Pérez, L.C., Schmidt, T., Mote, B., 2019. Multi-Pig Part Detection and Association with a Fully-Convolutional Network. Sensors (Basel, Switzerland) 19 (4). https://doi.org/10.3390/s19040852.

Ronneberger, O., Fischer, P., Brox, T., 2015. U-Net: Convolutional Networks for Biomedical Image Segmentation, in: Navab, N., Hornegger, J., Wells, W.M., Frangi, A.F. (Eds.), Medical image computing and computer-assisted intervention - MICCAI 2015: 18th international conference, Munich, Germany, October 5-9, 2015; proceedings, vol. 9351. Springer, Cham, pp. 234-241.

Rushen, J., Chapinal, N., am de Passillé, 2012. Automated monitoring of behavioural-based animal welfare indicators. Animal Welfare 21 (3), 339-350. https://doi.org/10.7120/09627286.21.3.339.

SAS R Institute Inc, 2008. User's guide (release 9.4). SASR Institute Inc.

Schultze, C., 2008. Enrichment bei der Haltung von Mastschweinen. Dissertation. München.

Statham, P., Green, L., Bichard, M., Mendl, M., 2009. Predicting tail-biting from behaviour of pigs prior to outbreaks. Applied Animal Behaviour Science 121 (3-4), 157-164. https://doi.org/10.1016/j.applanim.2009.09.011.

Stolba, A., Wood-Gush, D.G.M., 1989. The behaviour of pigs in a semi-natural environment. Animal Science 48 (2), 419-425. https://doi.org/10.1017/S0003356100040411.

Stukenborg, A., Traulsen, I., Puppe, B., Presuhn, U., Krieter, J., 2011. Agonistic behaviour after mixing in pigs under commercial farm conditions. Applied Animal Behaviour Science 129 (1), 28-35. https://doi.org/10.1016/j.applanim.2010.10.004.

Valros, A., 2018. Tail biting, in: Špinka, M. (Ed.), Advances in pig welfare. Woodhead Publishing an imprint of Elsevier, Duxford, United Kingdom, 137-166. 
van Putten, G., 1978. Komfortverhalten bei Schweinen und Seine Bedeutung fuer das Wohlbefinden der Tiere, in: Fölsch, D.W. (Ed.), The Ethology and Ethics of Farm Animal Production: Proceedings of the 28th Annual Meeting. Birkhäuser Basel, Basel, s.l., p. 129.

Wathes, C.M., Kristensen, H.H., Aerts, J.-M., Berckmans, D., 2008. Is precision livestock farming an engineer's daydream or nightmare, an animal's friend or foe, and a farmer's panacea or pitfall? Computers and Electronics in Agriculture 64 (1), 2-10. https://doi.org/10.1016/j.compag.2008.05.005.

Weary, D.M., Huzzey, J.M., Keyserlingk, M.A.G. von, 2009. Board-invited review: Using behavior to predict and identify ill health in animals. Journal of animal science 87 (2), 770-777. https://doi.org/10.2527/jas.2008-1297.

Wechsler, B., Schmid, H., Moser, H. (Eds.), 1991. Der Stolba-Familienstall für Hausschweine: Ein tiergerechtes Haltungssystem für Zucht- und Mastschweine. Birkhäuser Basel, Basel, s.l., 97 pp.

Wennrich, G. (Ed.), 1978. Anpassungsfähigkeit. Verlag Paul Parey, Berlin and Hamburg. 


\section{GENERAL Discussion}

The aim of the present dissertation was to evaluate the effect of different farrowing and rearing systems on tail lesions and losses of rearing and fattening pigs. The focus was to investigate the differences of docked and undocked animals. The general levels of tail lesions and losses of the different housing systems over time were related. Subsequently, pigs with differing intensities of tail lesions were compared and their specific characteristics were identified. A comparison of the long-term effects of artificial rearing and housing was conducted in pens with conventional farrowing crates during the nursery period with special emphasis on tail biting, skin lesions and performance traits. Finally, the level of activity behaviour during rearing was determined and an automatic and manual analysis method were compared.

\section{Scoring of pigs' tails}

Tail biting causes many welfare and health problems for pigs (D'Eath et al., 2014; Munsterhjelm et al., 2017). The extent of the risk factors and thus also their effects on primarily undocked animals varies between farms (Schrøder-Petersen and Simonsen, 2001). To assess the extent of tail biting, so-called scoring keys are used, as in Papers One, Two and Three, which mainly assessed tail lesions and losses. In order to facilitate the comparability of studies, it would be reasonable to use the same scoring key, but different scoring keys have been used by different working groups (Honeck et al., 2019). The so-called "Deutscher SchweineBoniturschlüssel" was developed for Germany at the end of May 2017 (FLI, 2017); it enables national comparability through a standardised scheme. There is no common solution for international comparability so far. The main difference between the scoring keys depends on whether tail lesions and losses are recorded separately (Abriel and Jais, 2013a; Naya et al., 2018; Veit et al., 2016b) or simultaneously as one measurement (e.g. small bite marks but tail is intact or medium-sized wounds, part of tail removed) (Statham et al., 2011; van Nieuwamerongen et al., 2017; van Nieuwamerongen et al., 2015). Considering the first tail biting event, it is useful to measure both parameters together because a severe lesion usually causes a loss and a minor lesion can normally heal without loss (Honeck et al., 2019). However, if tail biting occurs more than once, it is difficult to separate old and new lesions and losses if they have been assessed simultaneously before. Therefore, it is preferable to evaluate the development lesions and losses apart from each other. In order to determine the age of the lesion, the freshness of blood is frequently used (Honeck et al., 2019). This parameter is positively correlated with tail lesion, which means the more lesions the higher the likelihood of blood at the tail (Ursinus et al., 2014). Although this correlation renders the recording of both parameters redundant, the assessment of blood at the tail provides additional information on 
the age of the wound (Keeling et al., 2012). This knowledge enables the assessor to determine whether the biting is acute and happened just before the assessment or if the biting had happened in the recent past (Holling et al., 2017). Anyway, since freshness of blood can easily be seen and detected on the animal and pen, it can be a helpful tool for farmers to detect tail biting and to rate whether their intervention measures to prevent tail biting outbreaks have been successful (Zonderland et al., 2008). While distinguishing the different stages of tail biting, the assessment of blood is necessary to differentiate the first stage from the second by absence of blood (Fraser, 1987; Taylor et al., 2010; Zonderland et al., 2008). In the second stage, the tail shows severe bloody lesions resulting from "sudden-forceful" biting behaviour. Another additional parameter for scoring keys is tail posture (Larsen et al., 2016). The tail can be curled up, hanging or squeezed between the legs (Zonderland et al., 2009). Tail posture is strongly related to tail lesions at the same moment and a squeezed tail between the legs can predict tail lesions two - three days later (Zonderland et al., 2009). This means, scoring tail postures increases early recognition of tail biting (Zonderland et al., 2009).

The scientific question is deciding to select the appropriate scoring key because it determines the required degree of detail. Additionally, the parameters (tail lesions, losses, posture) to be investigated can be different. Different parameters lead to distinctive conclusions as tail lesions, losses and blood provide information on tail biting in general and tail posture can be useful to evaluate the stress level within group (Honeck et al., 2019). Furthermore, it is necessary to distinguish between complex analyses which correlate with detailed parameters (Statham et al., 2011), and studies which need to confirm whether tail biting has taken place or not (Calderón Díaz et al., 2018). The scoring method to assess the parameters can vary as well. For example, direct observation (Zonderland et al., 2008) as well as video observation (D'Eath et al., 2018). can be used for the detection of tail posture. Moreover, direct observation can be divided into evaluation from inside the pen (Statham et al., 2011; van Nieuwamerongen et al., 2017), where a detailed analysis can be conducted or from outside the pen, standing on the corridor, where a simple scoring key is needed (Scollo et al., 2017; Scollo et al., 2016). The present study (Papers One, Two and Three) used a modified Schwarzenauer key (Abriel and Jais, 2013b). The Schwarzenauer key measures tail lesions (by injured surface) and tail losses (in thirds) in steps (0-3), where 0 means no lesion respectively no loss and 3 stands for a large surface lesion respectively for a loss of more than two-thirds of the tail. In addition, swelling and fresh blood are recorded. Since there was no nationally standardised scoring key at the beginning of the trial in May 2016, this key was used. However, it was slightly modified by recording the losses in quarters and by recording necrosis in addition to swelling and blood, as is the practice in the "Deutscher Schweine-Boniturschlüssel". 
The complexity of the assessment is additionally dependant on the observer. Trained observers can use complex methods (Scollo et al., 2013) while farmers who rate the tails during their daily routines need simple scoring keys. Whether the assessment is made on an animal or pen basis and the degree of detail of the rating key can be significant. Sinisalo et al. (2010) found that the prevalence of tail biting is non-linearly related to the proportion of individual pigs with tail lesions. When $50 \%$ of the pens are considered to be affected by tail biting, only $32 \%$ of the pigs had lesions (Sinisalo et al., 2010). In conclusion, the scoring key needs to be optimised for the respective evaluation, the scientific question and the observer.

Another distinguishing feature is the frequency of scoring. In the present study (Papers One, Two and Three) the scoring was conducted weekly, so the development of tail lesions and losses was assessable. This interval was also selected by Veit et al. (2016b) and Naya et al. (2018), who also scored tail lesions and losses separately. In the literature, one can also find studies where the tail was checked twice a week, in this case smaller changes on the tail could be found (Abriel and Jais, 2013a). In trials which try to provide general group statements, an extended rhythm of four weeks is used for rating (Statham et al., 2009). There are also irregular scoring rhythms, which depend on the expected prevalence of tail biting. For example, Ursinus et al. (2014) scored weekly during the rearing period and every five weeks during fattening. This means that during the rearing period, more significant changes in the tail can be recorded and only a general statement can be made about the fattening period. The ability to define the period of tail biting is also limited by the frequency of the scoring. With weekly recording (Naya et al., 2018; Veit et al., 2016b) or an equally shorter interval (Abriel and Jais, 2013a), the time of the outbreak can be determined rather precisely. With a four-weekly observation (Statham et al., 2009), many wounds have healed (Baumgärtner and Gruber, 2015) and losses are the only way to determine if an outbreak has occurred in the meantime.

\section{Data analysis}

In the current study, generalised linear mixed models were used to evaluate tail lesions and losses (Papers One and Three). This is a common method for estimating the basic level of lesions or losses per time point including several fixed effects and has also been used in previous studies (Hunter et al., 2001; Lahrmann et al., 2017; Li et al., 2017; Naya et al., 2018; Veit et al., 2016b). The main advantage of this method is that effects can be considered separately, and the influence of individual parameters can thus be highlighted. However, this approach is difficult to apply if the effects cannot be separated from each other. The interpretation of confounded effects is complex because the effect of the individual parameter can be over- or underestimated. Other evaluations of similar issues have often used Anova or Chi Square Test (Herskin et al., 2015; Kritas and Morrison, 2004). Since these tests do not 
consider the effects on the animals, these methods were the methods of choice for the present study.

A survival analysis can be performed to determine when tail lesions occur for the first time and whether the risk of being bitten varies for potential victims in different weeks of life (Larsen et al., 2018). Survival analysis is a method that calculates the time until an event occurs, whereby the events can be, for example, diseases or death (Kleinbaum and Klein, 2012). For the current study, this method could not be used because multiple risk factors (e.g. docking status, housing system, sex) were involved in the occurrence of tail lesions respectively tail losses. The survival analysis assumes that risk factors are dependant or independent from each other (Kleinbaum and Klein, 2012). A definite division of the effects into dependent and independent factors was not possible in the present study. In addition, the number of animals differed in the rearing and fattening periods. A weekly calculation of risk would therefore have been appropriate for those animals tested over the 18 weeks. An experimental design in which the same animals could be observed over the entire time would favour this method.

The method of cluster analysis was chosen to separate individual characteristics of pigs without tail lesions or, in the other extreme scenario, with many lesions throughout their lives (Paper Two) (Hartigan and Wong, 1979; Sparks et al., 2012). A cluster analysis creates subsets of a group (clusters) which are supposed to be as homogeneous as possible within a group with regard to the set of cluster characteristics (Eckstein, 2016). A principal component analysis can also be used to work out specific group characteristics (Abdi and Williams, 2010). However, this can only be implemented mathematically if there is a complete rectangular matrix (Abdi and Williams, 2010), which means that all treatment groups must occur more than once in all combinations. In the current study, three farrowing systems, two rearing systems and two docking statuses were tested. Due to the fact that the same population of animals was not represented in the rearing and fattening periods and that certain test combinations only occurred once, this approach was not suitable and therefore not available in the present study.

For the examination of large datasets, which should be used to identify similarities of several farms, for example, a regression tree analysis might be suitable. Grümpel et al. (2018) used this method to extract risk factors from numerous pig farms. The authors were challenged by the diversity of the farms to find a statistical method to examine single outstanding risk factors applying to more than one farm. This method could not be used for the current study because only one farm was involved and the effects on the pigs were identical for all animals.

In summary, complex statistical analyses require a complete matrix with the replication of all experimental variations. For this purpose, a constant basic population is advantageous. 


\section{Video analysis of tail biting behaviour}

Pigs change their behaviour patterns prior to a tail biting outbreak (Statham et al., 2009). The most suitable method of non-invasive behavioural observation is the analysis of videos or recordings (Rushen et al., 2012). This method has advantages compared to direct observation, the animals could be distracted due to human presence (Rushen et al., 2012). Pigs are more active during daytime, so most tail biting is conducted in the light hours of the day (Fraser and Broom, 1990). Tail biting behaviour can be attributed to normal behaviour involving patterns as suckling, exploration, feeding or social behaviours but several types of tail biting result from different motivations (Schrøder-Petersen and Simonsen, 2001; Taylor et al., 2010). Tail biting may be detected and thus prevented by monitoring the animals and possible behavioural changes. Many studies have focused on tail biting victims (Naya et al., 2018; Veit et al., 2016b). However, a reduction in tail biting might be achieved by improving the knowledge of the biters (Beattie et al., 2005; Taylor et al., 2010), which could be achieved by using video analysis. Statham et al. (2009) were able to detect an increased activity level in biters as well as increased explorative behaviour. In the current experiment, video analysis was used to analyse the activity behaviour of rearing pigs. The results can be found in Paper Four. The most important advantage of video analysis is that it does not interfere with the normal behaviour of the animals (Rushen et al., 2012), compared to direct scoring of the tails, for example. Manual evaluation of the video recordings is time-consuming and expensive. Automatic analysis in contrast has a high accuracy and validity and it is also faster (Rushen et al., 2012). Nonetheless, currently it is not possible to automatically process all questions that can be answered by manual evaluation. However, this could be achieved in the future through technical progress and possibly also through a combination of manual and automatic methods.

\section{Characteristics of biters and victims}

Previous studies have examined the differences between biters and victims of tail biting. Biters are often smaller than other pigs in the pen, which could be related to the fact that they have a reduced feed intake or a decreased nutrient absorption and therefore their explorative behaviour becomes more pronounced (Schrøder-Petersen and Simonsen, 2001; van de Weerd et al., 2005). Pigs which are born smaller have a tendency to increased tail-in-mouth behaviour after weaning (Munsterhjelm et al., 2016). At the start of the fattening period, the biters are also the smallest animals in tendency (Sinisalo et al., 2012). Zonderland et al. (2011b) did not find a difference in size between tail biting performers and neutral animals but they showed that the victims of biting were larger than the biters. Tail biting could also be an alternative to agonistic interactions (Wallgren and Lindahl, 1996). Smaller animals are often inferior in normal fights, so they attack bigger pigs from behind (Fraser and Broom, 1990). 
Frustration caused by being pushed away from the feeding trough or by being removed from the sleeping place caused by the physical inferiority of smaller animals could be the reason for the abnormal behaviour (Sambraus, 1985). The current analysis focused on the victims of tail biting. Occasionally, biters were identified, but these were only rehoused and received no further attention.

\section{Time point of tail biting}

The age and the weight of pigs are influencing factors on the incidence of tail biting (SchrøderPetersen and Simonsen, 2001). While studying docked pigs, Sambraus (1985) found that tail biting only becomes a problem for pigs aged about 90-120 days and Blackshaw (1981) detected a mean age of 40.7 ( \pm 15.5$)$ days for the first outbreak. Nowadays, studies focus on undocked pigs and it is assumed that the pigs do not have any tail lesions before weaning and that tail biting is a general problem during the rearing and fattening period (Munsterhjelm et al., 2013; Zonderland et al., 2011b). However, the development of biting behaviour may be learned during the suckling period (Schrøder-Petersen et al., 2003) while piglets perform manipulation of their siblings (Cox and Cooper, 2001; Oostindjer et al., 2011). Tail lesions gained in the first weeks of life could be so small that they are hardly visible and therefore the general level of tail biting during the suckling period may be underestimated (Ursinus et al., 2014).

Previous studies have also focused on different periods during the pig's life to evaluate tail biting. Statham et al. (2009) and Ursinus et al. (2014) observed undocked pigs from suckling until the end of fattening, Zonderland et al. (2009) and Zonderland et al. (2011b) focused on the rearing period and Wallenbeck and Keeling (2013) only evaluated the fattening period. Depending on the frequency of scoring and on the period, different numbers of outbreaks have been detected, thus prevalence also varied. Statham et al. (2009) detected six, Zonderland et al. (2011b) found 14 and Wallenbeck and Keeling (2013) showed 21 outbreaks of tail biting during the respective assessment period. Due to the differences in the datasets, not only in the number of animals but also in the size of the groups, comparability is rather difficult. However, it should be noted that tail biting is not only a problem of the rearing period but can also occur during fattening. This is a reason why the periods from weaning to the end of fattening were evaluated in the present study.

\section{Handling of tail biting outbreaks}

Early detection of tail biting outbreaks can reduce impairment for pigs and farmer. Undocked pigs are housed on several farms but only some of them have tail biting problems because the risk of this abnormal behaviour can be reduced by intensive animal observation (Veit, 2016a). 
Initial signs of negative influencing factors for the pigs need to be identified and corrected. In literature, there are several studies which have described intervention methods for tail biting (Valros et al., 2016; Zonderland et al., 2011a). The identification and the removing of the biter has turned out to be an efficient measure to stop an outbreak (Valros et al., 2016). This method seems to be more useful than removing the victim with the tail lesion (Zonderland et al., 2011a) although there is not always just one tail biter and identification can be challenging (Zonderland et al., 2011a). The rehousing of the victims might be useful in cases of non-consistent tail biting in the pen and because of the increased interest in wounded tails (Ursinus et al., 2014). Another way of distracting the pigs is the adding of enrichment material (Hunter et al., 2001). In the present study, intervention was practised if an outbreak occurred. New enrichment material was provided to the pigs and the severely injured animals were medically treated. When the bitten pigs had recovered, they remained in the pen, but when they suffered, and the inflammation spread, they were removed and excluded from the experiment. Only in rare cases could biters be identified; if this was a single animal it was removed from the pen. However, this was not possible in the case of several biters.

\section{Risk factors of tail biting}

Tail biting is a multifactorial problem (Valros, 2018). The risk factors mentioned in the literature review were also relevant to the present study. With regard to the housing system, the flooring (Moinard et al., 2003; Schrøder-Petersen and Simonsen, 2001) as well as the enrichment material need to be considered (EFSA, 2007). In contrast to the conventionally reared animals, the wean-to-finish animals had concrete flooring from the time of weaning, whereas plastic flooring was used in the CONV system. Both flooring types do not provide the best conditions for the bedding of the animals, nevertheless, they are different. Concrete flooring is harder and may have sharp slats but it also stores and conducts heat, which might be more comfortable for the pigs. Plastic is more flexible but creates louder background noise. The provision of enrichment material was equal for all pigs. Both effects were confounded in the effect of the rearing system and thus were not measured separately. The influences of feeding, such as for example detected by Hunter et al. (2001), could not be considered here, since all test groups were fed the same diet. All pigs received the feed in pellet form, so that the risk of different ingredients, as with liquid feed, could not occur (Palander, 2016). Zonderland et al. (2010) showed differences of the sexes; this was also detected in the present study as males suffered more tail lesions and losses. Castrated animals were not included in the experiment and therefore no comparison can be established. Due to the consistent use of the same genetics, there have been no findings in this specific area. But most studies use cross breeding in their trials (Herskin et al., 2015; Naya et al., 2018; Veit, 2016a). Maybe attempts with pure breeding would be interesting in the future. 


\section{Conclusion}

In conclusion, the housing system plays a particularly important role during the rearing period in influencing tail biting behaviour, with long-term effects on tail lesions and losses. Increased space allowance and a reduced number of regroupings, as with the wean-to-finish pigs, appears to have a positive effect in reducing lesions. There remains a large difference in the number and severity of tail lesions and losses between docked and undocked pigs. Despite animal welfare concerns, artificially reared pigs benefit from maintaining stable groups and exhibit fewer tail lesions and losses, fewer skin lesions, and their performance is comparable to pigs reared conventionally. Fewer regroupings, more space during rearing and therefore less stress due to not needing to form new hierarchies contribute to less tail biting. Furthermore, the automatic behaviour analysis has shown to be able to successfully separate active and inactive time intervals at group level. It promises a high potential, especially regarding early warning indicators for tail biting.

\section{References}

Abdi, H., Williams, L.J., 2010. Principal component analysis. Wiley Interdisciplinary Reviews: Computational Statistics 2 (4), 433-459. https://doi.org/10.1002/wics.101.

Abriel, M., Jais, C., 2013a. Einfluss der Haltungsbedingungen auf das Auftreten von Kannibalismus bei Aufzuchtferkeln. Landtechnik (68 (6)), 389-394.

Abriel, M., Jais, C., 2013b. Mehr Tierwohl - Maßnahmen im Bereich der Haltung: Versuche zur Reduzierung des Schwanzbeißens bei Ferkeln 11, 41-42. Bayrische Landesanstalt für Landwirtschaft, Freising, 95 pp.

Baumgärtner, W., Gruber, A.D. (Eds.), 2015. Allgemeine Pathologie für die Tiermedizin, 2nd ed. Enke, Stuttgart, $292 \mathrm{pp}$.

Beattie, V.E., Breuer, K., O'Connell, N.E., Sneddon, I.A., Mercer, J.T., Rance, K.A., Sutcliffe, M.E.M., Edwards, S.A., 2005. Factors identifying pigs predisposed to tail biting. Animal Science 80 (3), 307-312. https://doi.org/10.1079/ASC40040307.

Blackshaw, J.K., 1981. Some behavioural deviations in weaned domestic pigs: persistent inguinal nose thrusting, and tail and ear biting. Animal Science 33 (3), 325-332. https://doi.org/10.1017/S000335610003172X.

Calderón Díaz, J.A., García Manzanilla, E., Diana, A., Boyle, L.A., 2018. Cross-Fostering Implications for Pig Mortality, Welfare and Performance. Frontiers in veterinary science 5, 123. https://doi.org/10.3389/fvets.2018.00123. 
Cox, L.N., Cooper, J.J., 2001. Observations on the pre- and post-weaning behaviour of piglets reared in commercial indoor and outdoor environments. Animal Science 72 (01), 75-86. https://doi.org/10.1017/S1357729800055570.

D'Eath, R.B., Arnott, G., Turner, S.P., Jensen, T., Lahrmann, H.P., Busch, M.E., Niemi, J.K., Lawrence, A.B., Sandøe, P., 2014. Injurious tail biting in pigs: how can it be controlled in existing systems without tail docking? Animal 8 (9), 1479-1497. https://doi.org/10.1017/S1751731114001359.

D'Eath, R.B., Jack, M., Futro, A., Talbot, D., Zhu, Q., Barclay, D., Baxter, E.M., 2018. Automatic early warning of tail biting in pigs: 3D cameras can detect lowered tail posture before an outbreak. PloS one 13 (4), e0194524. https://doi.org/10.1371/journal.pone.0194524.

Eckstein, P.P., 2016. Statistik für Wirtschaftswissenschaftler: Eine realdatenbasierte Einführung mit SPSS, 5th ed. Springer Gabler, Wiesbaden, 460 pp.

EFSA, 2007. European Food Safety Authority: Scientific Opinion of the Panel on Animal Health and Welfare on a request from commission on the risks associated with tail biting in pigs and possible means to reduce the need for tail docking considering the different housing and husbandry systems, EFSA J. 611, 1-13.

FLI, 2017. Friedrich-Loeffler-Institut: Deutscher Schweine Boniturschlüssel. https://www.fli.de/de/institute/institut-fuer-tierschutz-und-tierhaltungitt/forschungsbereiche-arbeitsgruppen/ag-schweine/deutscher-schweineboniturschluessel/.

Fraser, A.F., Broom, D.M., 1990. Farm animal behaviour and welfare, 3rd ed. CAB International, Wallingford, Oxon, UK, 437 pp.

Fraser, D., 1987. Attraction to blood as a factor in tail-biting by pigs. Applied Animal Behaviour Science 17 (1-2), 61-68. https://doi.org/10.1016/0168-1591(87)90008-6.

Grümpel, A., Krieter, J., Veit, C., Dippel, S., 2018. Factors influencing the risk for tail lesions in weaner pigs (Sus scrofa). Livestock Science 216, 219-226. https://doi.org/10.1016/j.livsci.2018.09.001.

Hartigan, J.A., Wong, M.A., 1979. Algorithm AS 136: A K-Means Clustering Algorithm. Applied Statistics 28 (1), 100. https://doi.org/10.2307/2346830.

Herskin, M.S., Thodberg, K., Jensen, H.E., 2015. Effects of tail docking and docking length on neuroanatomical changes in healed tail tips of pigs. Animal 9 (4), 677-681. https://doi.org/10.1017/S1751731114002857.

Holling, C., grosse Beilage, E., Vidondo, B., Nathues, C., 2017. Provision of straw by a foraging tower -effect on tail biting in weaners and fattening pigs. Porcine health management 3, 4. https://doi.org/10.1186/s40813-017-0052-7. 
Honeck, A., Gertz, M., grosse Beilage, E., Krieter, J., 2019. Comparison of different scoring keys for tail-biting in pigs to evaluate the importance of one common scoring key to improve the comparability of studies - A review. Applied Animal Behaviour Science 221, 104873. https://doi.org/10.1016/j.applanim.2019.104873.

Hunter, E.J., Jones, T.A., Guise, H.J., Penny, R.H., Hoste, S., 2001. The relationship between tail biting in pigs, docking procedure and other management practices. Veterinary journal 161 (1), 72-79. https://doi.org/10.1053/tvjl.2000.0520.

Keeling, L.J., Wallenbeck, A., Larsen, A., Holmgren, N., 2012. Scoring tail damage in pigs: an evaluation based on recordings at Swedish slaughterhouses. Acta veterinaria Scandinavica 54, 32. https://doi.org/10.1186/1751-0147-54-32.

Kleinbaum, D.G., Klein, M., 2012. Survival Analysis: A Self-Learning Text, Third Edition. Springer Science+Business Media LLC, New York, NY.

Kritas, S.K., Morrison, R.B., 2004. An observational study on tail biting in commercial growerfinisher barns. Journal of Swine Health and Production (12), 17-22.

Lahrmann, H.P., Busch, M.E., D'Eath, R.B., Forkman, B., Hansen, C.F., 2017. More tail lesions among undocked than tail docked pigs in a conventional herd. Animal 11 (10), 1825-1831. https://doi.org/10.1017/S1751731117000490.

Larsen, M.L.V., Andersen, H.M.-L., Pedersen, L.J., 2016. Can tail damage outbreaks in the pig be predicted by behavioural change? Veterinary journal 209, 50-56.

https://doi.org/10.1016/j.tvjl.2015.12.001.

Larsen, M.L.V., Andersen, H.M.-L., Pedersen, L.J., 2018. Which is the most preventive measure against tail damage in finisher pigs: tail docking, straw provision or lowered stocking density? Animal 12 (6), 1260-1267. https://doi.org/10.1017/S175173111700249X.

Li, Y.Z., Zhang, H.F., Johnston, L.J., Martin, W., Peterson, J.D., Coetzee, J.F., 2017. Effects of tail docking and tail biting on performance and welfare of growing-finishing pigs in a confinement housing system. Journal of Animal Science 95 (11), 4835-4845. https://doi.org/10.2527/jas2017.1571.

Moinard, C., Mendl, M., Nicol, C.J., Green, L.E., 2003. A case control study of on-farm risk factors for tail biting in pigs. Applied Animal Behaviour Science (81 (4)), 333-355. https://doi.org/10.1016/S0168-1591(02)00276-9.

Munsterhjelm, C., Heinonen, M., Valros, A., 2016. Can tail-in-mouth behaviour in weaned piglets be predicted by behaviour and performance? Applied Animal Behaviour Science 184, 16-24. https://doi.org/10.1016/j.applanim.2016.08.013.

Munsterhjelm, C., Nordgreen, J., Aae, F., Heinonen, M., Olstad, K., Aasmundstad, T., Janczak, A.M., Valros, A., 2017. To be blamed or pitied? The effect of illness on social 
behavior, cytokine levels and feed intake in undocked boars. Physiology \& behavior 179, 298-307. https://doi.org/10.1016/j.physbeh.2017.06.024.

Munsterhjelm, C., Simola, O., Keeling, L., Valros, A., Heinonen, M., 2013. Health parameters in tail biters and bitten pigs in a case-control study. Animal 7 (5), 814-821. https://doi.org/10.1017/S1751731112002194.

Naya, A., Traulsen, I., Gertz, M., Hasler, M., Burfeind, O., große Beilage, E., Krieter, J., 2018. Is tail biting in growing pigs reduced by a prolonged suckling period? Applied Animal Behaviour Science. https://doi.org/10.1016/j.applanim.2018.10.020.

Oostindjer, M., van den Brand, H., Kemp, B., Bolhuis, J.E., 2011. Effects of environmental enrichment and loose housing of lactating sows on piglet behaviour before and after weaning. Applied Animal Behaviour Science 134 (1-2), 31-41. https://doi.org/10.1016/j.applanim.2011.06.011.

Palander, P.A., 2016. The tail biting pig - Nutritional and physiological approaches to understanding the behavior. PhD Thesis. Helsinki, Finland, $88 \mathrm{pp}$.

Rushen, J., Chapinal, N., am de Passillé, 2012. Automated monitoring of behavioural-based animal welfare indicators. Animal Welfare 21 (3), 339-350. https://doi.org/10.7120/09627286.21.3.339.

Sambraus, H.H., 1985. Mouth-based anomalous syndromes: Ethology of Farm Animals. A Comprehensive Study of the Behavioural Features of Common Farm Animals. World Animal Science A5, 391-422.

Schrøder-Petersen, D.L., Simonsen, H.B., 2001. Tail biting in pigs. Veterinary journal 162 (3), 196-210. https://doi.org/10.1053/tvjl.2001.0605.

Schrøder-Petersen, D.L., Simonsen, H.B., Lawson, L.G., 2003. Tail-in-mouth Behaviour Among Weaner Pigs in Relation to Age, Gender and Group Composition Regarding Gender. Acta Agriculturae Scandinavica, Section A - Animal Science 53 (1), 29-34. https://doi.org/10.1080/09064700310002017.

Scollo, A., Contiero, B., Gottardo, F., 2016. Frequency of tail lesions and risk factors for tail biting in heavy pig production from weaning to $170 \mathrm{~kg}$ live weight. Veterinary journal 207 , 92-98. https://doi.org/10.1016/j.tvjl.2015.10.056.

Scollo, A., Di Martino, G., Bonfanti, L., Stefani, A.L., Schiavon, E., Marangon, S., Gottardo, F., 2013. Tail docking and the rearing of heavy pigs: the role played by gender and the presence of straw in the control of tail biting. Blood parameters, behaviour and skin lesions. Research in veterinary science 95 (2), 825-830. https://doi.org/10.1016/j.rvsc.2013.06.019.

Scollo, A., Gottardo, F., Contiero, B., Edwards, S.A., 2017. A cross-sectional study for predicting tail biting risk in pig farms using classification and regression tree analysis. 
Preventive veterinary medicine 146, 114-120.

https://doi.org/10.1016/j.prevetmed.2017.08.001.

Sinisalo, A., Niemi, J.K., Heinonen, M., Valros, A., 2012. Tail biting and production performance in fattening pigs. Livestock Science 143 (2-3), 220-225. https://doi.org/10.1016/j.livsci.2011.09.019.

Sinisalo, A., Niemi, J.K., Valros, A., 2010. Dynamic pattern, consequences and economic importance of tail-biting epidemics in fattening pigs. Proceedings of 44th Congress of the International Society for Applied Ethology, 2010, Uppsala Sweden 4-9th August 2010.

Sparks, R.L., Patton, J., Ganschow, L., 2012. Profiles of more and less successful L2 learners: A cluster analysis study. Learning and Individual Differences 22 (4), 463-472. https://doi.org/10.1016/j.lindif.2012.03.009.

Statham, P., Green, L., Bichard, M., Mendl, M., 2009. Predicting tail-biting from behaviour of pigs prior to outbreaks. Applied Animal Behaviour Science 121 (3-4), 157-164. https://doi.org/10.1016/j.applanim.2009.09.011.

Statham, P., Green, L., Mendl, M., 2011. A longitudinal study of the effects of providing straw at different stages of life on tail-biting and other behaviour in commercially housed pigs. Applied Animal Behaviour Science 134 (3-4), 100-108. https://doi.org/10.1016/j.applanim.2011.08.009.

Taylor, N.R., Main, D.C.J., Mendl, M., Edwards, S.A., 2010. Tail-biting: A new perspective. Veterinary journal 186 (2), 137-147. https://doi.org/10.1016/j.tvjl.2009.08.028.

Ursinus, W.W., van Reenen, C.G., Kemp, B., Bolhuis, J.E., 2014. Tail biting behaviour and tail damage in pigs and the relationship with general behaviour: Predicting the inevitable? Applied Animal Behaviour Science 156, 22-36. https://doi.org/10.1016/j.applanim.2014.04.001.

Valros, A., 2018. Tail biting, in: Špinka, M. (Ed.), Advances in pig welfare. Woodhead Publishing an imprint of Elsevier, Duxford, United Kingdom, 137-166.

Valros, A., Munsterhjelm, C., Hänninen, L., Kauppinen, T., Heinonen, M., 2016. Managing undocked pigs - on-farm prevention of tail biting and attitudes towards tail biting and docking. Porcine health management 2, 2. https://doi.org/10.1186/s40813-016-0020-7. van de Weerd, H.A., Docking, C.M., Day, J.E.L., Edwards, S.A., 2005. The development of harmful social behaviour in pigs with intact tails and different enrichment backgrounds in two housing systems. Animal Science 80 (3), 289-298. https://doi.org/10.1079/ASC40450289.

van Nieuwamerongen, S.E., Soede, N.M., van der Peet-Schwering, C.M.C., Kemp, B., Bolhuis, J.E., 2015. Development of piglets raised in a new multi-litter housing system vs. conventional single-litter housing until 9 weeks of age. Journal of Animal Science 93 (11), 5442-5454. https://doi.org/10.2527/jas.2015-9460. 
van Nieuwamerongen, S.E., Soede, N.M., van der Peet-Schwering, C.M.C., Kemp, B., Bolhuis, J.E., 2017. Gradual weaning during an extended lactation period improves performance and behavior of pigs raised in a multi-suckling system. Applied Animal Behaviour Science 194, 24-35. https://doi.org/10.1016/j.applanim.2017.05.005.

Veit, C., 2016a. Influence of raw material and weaning management on the occurrence of tail-biting in undocked pigs. PhD Thesis. Hannover.

Veit, C., Traulsen, I., Hasler, M., Tölle, K., Burfeind, O., grosse Beilage, E., Krieter, J., 2016b. Influence of raw material on the occurrence of tail-biting in undocked pigs. Livestock Science 191, 125-131. https://doi.org/10.1016/j.livsci.2016.07.009.

Wallenbeck, A., Keeling, L.J., 2013. Using data from electronic feeders on visit frequency and feed consumption to indicate tail biting outbreaks in commercial pig production. Journal of Animal Science 91 (6), 2879-2884. https://doi.org/10.2527/jas.2012-5848.

Wallgren, P., Lindahl, E., 1996. The influence of tail biting on performance of fattening pigs. Acta Vet Scand 37, 453-460.

Zonderland, J.J., Bracke, M.B.M., den Hartog, L.A., Kemp, B., Spoolder, H.A.M., 2010. Gender effects on tail damage development in single- or mixed-sex groups of weaned piglets. Livestock Science 129 (1-3), 151-158. https://doi.org/10.1016/j.livsci.2010.01.018.

Zonderland, J.J., Kemp, B., Bracke, M.B.M., den Hartog, L.A., Spoolder, H.A.M., 2011a. Individual piglets' contribution to the development of tail biting. Animal 5 (4), 601-607. https://doi.org/10.1017/S1751731110002132.

Zonderland, J.J., Schepers, F., Bracke, M.B.M., den Hartog, L.A., Kemp, B., Spoolder, H.A.M., 2011b. Characteristics of biter and victim piglets apparent before a tail-biting outbreak. Animal 5 (5), 767-775. https://doi.org/10.1017/S1751731110002326.

Zonderland, J.J., van Riel, J.W., Bracke, M.B.M., Kemp, B., den Hartog, L.A., Spoolder, H.A.M., 2009. Tail posture predicts tail damage among weaned piglets. Applied Animal Behaviour Science 121 (3-4), 165-170. https://doi.org/10.1016/j.applanim.2009.09.002.

Zonderland, J.J., Wolthuis-Fillerup, M., van Reenen, C.G., Bracke, M.B.M., Kemp, B., Hartog, L.A.d., Spoolder, H.A.M., 2008. Prevention and treatment of tail biting in weaned piglets. Applied Animal Behaviour Science 110 (3-4), 269-281.

https://doi.org/10.1016/j.applanim.2007.04.005. 


\section{SUMMARY}

Tail biting is a widespread behavioural disorder in modern pig housing systems. It is defined as an oral manipulation of a pig's tail by another. Different types of tail biting are described throughout the literature which are discernible by the way of biting as well as multifactorial causes. Due to the prohibition of routine tail docking in the EU, adapted housing systems for undocked pigs have gained importance.

The aim of this dissertation is to investigate the effect of farrowing and rearing systems on tail lesions and losses of pigs, with a focus on undocked animals. Different housing systems, as described below, are taken into consideration as a means to evaluate their influence on behaviour abnormalities during rearing and fattening periods.

In Paper One, docked and undocked pigs are compared regarding their tail lesions and losses during rearing and fattening periods. The pigs are housed in three farrowing systems: conventional farrowing crates (FC); free farrowing pens (FF); and group housing of lactating sows $(\mathrm{GH})$. Additionally, they are divided into two rearing systems: a conventional system (CONV), where the pigs were regrouped and transferred to conventional finishing pens at ten weeks of age; or a wean-to-finish (W-F) system, where the pigs remained in their pens until slaughter with increased space allowance during rearing. Significant differences in the rearing systems are observed through the weekly assessment of tail lesions and losses. Fewer lesions occur for the W-F pigs (maximum: CONV $58.01 \%$, W-F $41.16 \%$ ). Docked animals also experience statistically significantly fewer lesions than undocked animals. The W-F reared pigs have significantly fewer tail losses, as recorded at the end of fattening period (CONV $67.63 \%$, W-F $38.2 \%$ ). In conclusion, a long-term effect of early socialisation does not appear to influence tail biting, given that farrowing system does not have a statistically significant effect on tail lesions. Nonetheless, an increased space allowance and reduced regroupings included in the W-F rearing system decreases the frequency of tail lesions and losses, significantly.

Due to the findings of paper one, pigs with tail lesions were classified and characterised based on the frequency and duration of the lesion to create a latent parameter. Paper Two investigates whether pigs with different severity of tail lesions can be separated into biologically relevant groups using a cluster analysis. Pigs housed in FC, FF, GH and reared in CONV and $\mathrm{W}-\mathrm{F}$ are included in the analysis. Animals are indicated according to five lesion groups: from (I) no lesions to (V) many long lasting lesions; the separability of the predefined lesion groups is checked by an animal individual lesion parameter. As docking status is the main parameter affecting tail lesions, it is also included in the analysis. Using a k-means cluster analysis, groups I and V, are clearly distinguishable; however, the middle lesion groups are less defined 
within the analysis. In conclusion, not all pigs are affected by tail biting to the same extent. Docking status as well as increased space allowance can lead to a reduction in tail lesions.

Paper Three examines the long-term effects of artificial rearing (AR) on tail biting and development of pigs. AR and FC pigs are compared with a focus on tail lesions and losses, skin lesions, and performance traits. In this study, all pigs are raised in a conventional rearing system. Results show that AR pigs exhibit fewer lesions than the FC pigs; less than $15 \%$ of AR pigs had tail lesions. At the end of fattening period, only $6.9 \%$ of the AR pigs had lost parts of their tails, which was significantly different from FC pigs. AR pigs also have significantly fewer skin lesions after rehousing to rearing. After regrouping and rehousing to fattening, skin lesion frequency is not differing. Performance traits differ during nursery period in favour of the FC pigs, but in contrast, AR pigs have the higher daily weight gains during rearing period. Fattening period does not have a significant effect. AR pigs exhibit fewer tail lesions and losses with comparable daily weight gains, which is a result of reduced regroupings and identical housing conditions during rearing and fattening periods. However, these results should be critically reflected in terms of animal welfare and economic feasibility, prior to making practical recommendations.

Paper Four focusses on the activity behaviour of pigs. Animals' behaviours are recorded and evaluated manually and automatically at three time points during the rearing period (beginning, middle, end). The manual analysis uses the point sampling method and determines the respective activity of every pig every 12 minutes. The automatic evaluation is based on a computer vision algorithm and records the pigs as ellipses. The coordinates as well as the centers of the individual animal ellipses are measured. By linking the nearest neighbour ellipses, the movement of the pigs is recorded within 50 frames, adjusted to the manual time samples. The evaluation is carried out at pen level. The results show that the manual animal detection is more precise, but the automated analysis has a high accuracy and is only limited by objects that obscure the pigs. Humans can interpret the animals' behaviour, even though single body parts are not visible, whereas automatic analysis systems have difficulties in adapting this human knowledge about the shape and movements of animal bodies. Nonetheless, the daily course of the manually and automatically assessed activity and the trends of increasing or decreasing activity are comparable. Furthermore, days can be automatically divided into inactive, active and very active phases with a high accuracy. Overall, there is a high level of agreement between the two methods of analysis.

In summary, it can be concluded that the housing system plays a particularly important role during the rearing period in influencing tail biting behaviour, with long-term effects on tail lesions and losses. Increased space allowance and reduced number of regroupings, as with the $\mathrm{W}-\mathrm{F}$ pigs, appears to have a positive effect in reducing lesions. There remains a large 
difference in the number and severity of tail lesions and losses between docked and undocked pigs. Characteristics of pigs with and without tail lesions are identifiable using a cluster analysis. Despite animal welfare concerns, artificially reared pigs benefit from maintaining stable groups and exhibit fewer tail lesions and losses, less skin lesions, and their performance is comparable to pigs reared more traditionally. Fewer regroupings, more space during rearing and therefore less stress due to not needing to form new hierarchies contribute to less tail biting. Furthermore, the automatic behaviour analysis has shown to be able to successfully separate active and inactive time intervals at a group level. It promises a high potential, especially regarding early warning indicators for tail biting. 


\section{DANKSAGUNG}

Vielen Dank an alle, die zur Umsetzung und zum Gelingen dieser Arbeit beigetragen haben.

Herzlichen Dank an Frau Prof. Dr. Imke Traulsen für die wissenschaftliche Unterstützung, die produktiven Diskussionen und die Überlassung des interessanten Themas. Zudem möchte ich mich für die Möglichkeit bedanken, dass ich meine Ergebnisse im In- und Ausland präsentieren konnte. Ich habe während meiner Promotion sehr viel gelernt, vielen Dank für die spannende Zeit.

Mein Dank gilt Herrn Prof. Dr. Jürgen Hummel und Frau Prof. Dr. Eva Schlecht für die Übernahme des Co-Referats und für die konstruktiven Betreuungsausschusstreffen.

Dem Lehr- und Versuchszentrum Futterkamp danke ich für die gute Zusammenarbeit und die Hilfe während der Datenaufnahme und auch danach. Mein Dank gilt vor allem Harm Kruse und Dr. Onno Burfeind.

Die Förderung des Projektes erfolgte aus Mitteln des Zweckvermögens des Bundes bei der Landwirtschaftlichen Rentenbank (2817205413; 758914), bei denen ich mich ganz herzlich bedanken möchte. Ebenso gilt mein Dank der H. Wilhelm Schaumann Stiftung, die meine Doktorarbeit durch ein Promotionsstipendium unterstützt hat.

Ich möchte mich auch bei meiner Arbeitsgruppe für die Unterstützung bedanken. Vor allem danke ich Sebastian und Conny für die Diskussionen über statistische Methoden und die vielen Ratschläge zur Datenauswertung. Erwin danke ich für die stetige Hilfsbereitschaft. Meinen MitDoktoranden danke ich für die vielen Gespräche zwischendurch und für das Korrekturlesen.

Außerdem möchte ich mich bei Anita bedanken. Danke für die Unterstützung und Hilfsbereitschaft, die stundenlangen SAS-Fehlersuchen, die spontanen Telefondiskussionen und das geduldige Korrekturlesen. Natürlich möchte ich mich auch bei meinem zweiten Lieblings-InnoPig-Kollegen Michael bedanken. Ohne Euch hätte die Datenaufnahme nur halb so viel Spaß gemacht.

Vielen Dank an alle meine Freunde. Ein Dankeschön an meine Mensabegleiter, die sich selbst beim Mittagessen mit den Herausforderungen einer Promotion auseinandersetzen mussten. Danke an Daniela für den stetigen Beistand und die gute Unterhaltung auf den vielen Autofahrten.

Danke an Sassi für die immerwährende Motivation, die positive Sicht auf die Dinge und die Unterstützung. Danke für Deine Hilfe, wann immer ich sie brauchte.

Mein besonderer Dank gilt meiner Familie. Danke an meine Eltern, die für mein Interesse an der Landwirtschaft verantwortlich sind und mich immer unterstützt haben. Ein großer Dank geht auch an meine beiden Geschwister, die mich immer wieder mit der nötigen Portion Humor den Stress vergessen lassen haben.

Danke für die letzten drei Jahre! 


\section{EIDESSTATTLICHE ERKLÄRUNG}

Ich erkläre, dass diese Arbeit weder in gleicher noch in ähnlicher Form bereits anderen Prüfungsbehörden vorgelegen hat.

Weiter erkläre ich, dass ich mich an keiner anderen Hochschule um einen Doktorgrad beworben habe.

Göttingen, den 25. Mai 2020

Maria Gentz

Ich erkläre eidesstattlich, dass diese Dissertation selbständig und ohne unerlaubte Hilfe angefertigt wurde.

Göttingen, den 25. Mai 2020

Maria Gentz 